\title{
Quark-antiquark composite systems: the Bethe-Salpeter equation in the spectral-integration technique
}

\author{
A.V. Anisovich, V.V. Anisovich, V.N. Markov, \\ M.A. Matveev ,and A.V. Sarantsev
}

\begin{abstract}
The Bethe-Salpeter equations for the quark-antiquark composite systems, $q \bar{q}$, are written in terms of spectral integrals. For the $q \bar{q}$-mesons characterized by the mass $M$, spin $J$, and radial quantum number $n$, the equations are presented for the following $\left(n, M^{2}\right)$-trajectories: $\pi_{J}, \eta_{J}, a_{J}, f_{J}, \rho_{J}, \omega_{J}$, $h_{J}$, and $b_{J}$.
\end{abstract}

\section{INTRODUCTION}

The relativistic description of composite systems was always an actual and challenging task. The most frequently used technique, which takes into account the relativism of the constituents, is the Bethe-Salpeter equation [1]. But in the standard formulation of the Bethe-Salpeter equation, when the Feynman integration technique with mass-offshell amplitudes is used, one faces problems of the description of multiparticle channels and high-spin states. More appropriate technique for the high-spin composite systems is the dispersion relation approach, in particular, the most developed $N / D$-method [2]. However, our experience and intuition are based on the consideration of the potential type interactions, i.e., those which are associated with the particle-exchange mechanism. In terms of the $N / D$-method, one can easily relate the nearest left-hand side singularity of the $N$-function to the $t$-channel (or $u$-channel) mesonexchange diagram, but the reconstruction of the full set of left-hand singularities, when the interaction is given by the particle exchange or potential forces, is not a simple problem. Here we present the Bethe-Salpeter equation in terms of the spectral-integral technique which has advantages of both approaches discussed above:

(i) in the spectral integrals, the mass-on-shell amplitudes are used,

(ii) the interaction terms are written in the potential or particle-exchange form.

Moreover, in the spectral-integral technique one can use the energy-dependent forces as well.

In [3-5], the dispersion-relation approach was applied to the description of the deuteron, being two-nucleon composite system, by treating nucleon-nucleon forces in terms of separable interactions. By using the interaction in a separable form one can work with mass-on-shell amplitudes and meson-exchange interactions. The expansion of the one-meson exchange diagrams in a series of separable interaction factors was developed in [6]. The principal points in the transformation of the standard Bethe-Salpeter equation to the dispersion-relation representation for the case of separable vertices were clarified in [7]. However, the representation of the meson-exchange diagram as a finite set of the separable vertices works successfully for mesons with nonzero mass only. For the long-range interaction, like confinement forces, the separable-vertex approach fails, thus forcing us to use not the standard $N / D$-method but the spectral-integral technique.

The important ingredient of the dispersion relation and spectral-integration methods is the moment-operator expansion. The elements of the moment-operator expansion technique were presented in $[3, ?, 8]$, and a systematic presentation of the technique may be found in [9].

The Bethe-Salpeter equation in the spectral integral representation is written here for quark-antiquark systems. Our attention is focused on the light-quark bound states, $q \bar{q}$, where $q=u, d, s$ : for the simplicity's sake we consider here the systems built by quark and antiquark with equal masses: $u \bar{d}, u \bar{u}, d \bar{d}, s \bar{s}$. The treatment of heavy-quark composite systems, $c \bar{c}$ and $b \bar{b}$, can be performed similarly.

The necessity to deal with a full set of equations for the light-quark composite systems is governed by the rich information on the light-meson radiative decays that appeared recently [10-12]. The radiative decay data make it possible to restore the wave functions of mesons involved into reactions. Corresponding relativistic technique based on the consideration of the form-factor amplitudes in terms of the double spectral integrals was developed in [13] for pseudoscalar $q \bar{q}$-mesons, and it was generalized for scalar and tensor $q \bar{q}$-mesons in [14]. Finding out the meson wave functions in the spectral-integral form (or in the light-cone variables, see $[13, ?]$ for details) opens the way for direct reconstruction of the quark-antiquark interactions. The spectral-integral representation of the Bethe-Salpeter equation gives us the possibility to find out directly the interaction forces, provided the masses and wave functions of composite systems are known: this problem is discussed in Sect. 2 by using a simplified example of composite particles with spinless constituents.

For the reconstruction of $q \bar{q}$ forces it is important for the light-quark $q \bar{q}$-states to lay on linear trajectories in the $\left(n, M^{2}\right)$ plane, where $n$ is the radial quantum number of the meson with mass $M$ [15]. In more detail, the $q \bar{q}$-states can be classified, within spectroscopic notations, as the $n^{2 S+1} L_{J}$ levels, where $S, L$, and $J$ refer to the spin, orbital, 
and total momenta, respectively. The analysis of spectra in the mass region 1950-2400 MeV performed in [16] had fixed more than thirty new mesons which belong to the meson groups with positive and negative charge parities, $(C=+/-)$. Namely,for the $(C=+)$-states one has:

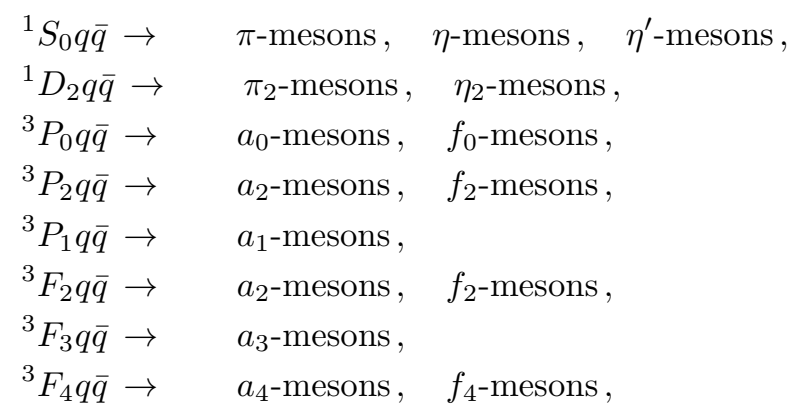

and for the $(C=-)$-states:

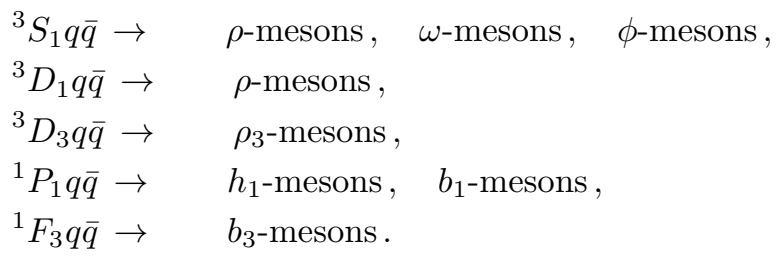

The mesons measured in [16] as well as those accumulated in the compilation [17], being classified versus radial quantum number $n$, can be put, with sufficiently good accuracy, on linear trajectories in the $\left(n, M^{2}\right)$-plane:

$$
M^{2}=M_{0}^{2}+\mu^{2}(n-1), \quad n=1,2,3,4, \ldots,
$$

with the universal slope $\mu^{2} \simeq 1.3 \mathrm{GeV}^{2}[15]$. The linearity of trajectories, leading and daughter ones, was observed for the $\left(J, M^{2}\right)$ plane too $[15]$.

The linearity of trajectories on the $\left(n, M^{2}\right)$ and $\left(J, M^{2}\right)$ planes is in a good agreement with large- $r$ behaviour of the confinement potential, $V(r) \sim \alpha r$, e.g., see [7], where the calculation of $q \bar{q}$ states from the groups (1) and (2) has been carried out.

At the same time it is necessary to emphasize that for the low-mass states one can expect a violation of the trajectory linearity. For example, the $\pi$-meson is just an exception that is not surprising because of a particular role of the pion. The standard explanation is that the pion, being a low-mass particle, is determined by the instanton-induced forces, see $[18,19]$ and references therein, although one cannot exclude an alternative modelling of the short-range forces. The problem of short-range forces is stressed by systematics of scalar states: the $K$-matrix analysis of $\pi \pi, K \bar{K}, \eta \eta$, and $\eta \eta^{\prime}$ spectra [20] tells us that the lightest scalar-isoscalar state belongs to the flavor octet, but in the model calculations $[18,19]$ the lightest state is close to the flavor singlet. We hope that a precise reconstruction of the $q \bar{q}$ forces can be facilitated by using the Bethe-Salpeter equation for the $q \bar{q}$ states in the spectral-integral form.

So, we focus our attention on the reconstruction of the $q \bar{q}$ interaction, on the basis of the following triad:

(1) the Bethe-Salpeter equation in the spectral-integral form,

(2) the linearity of trajectories on the $\left(n, M^{2}\right)$ and $\left(J, M^{2}\right)$ planes,

(3) the use of wave functions for low- and moderate-mass $q \bar{q}$ states found in the study of the meson radiative decays.

The important point is that radiative decays can give us the information about meson wave functions which are now studied in the mass region 1000-1800 MeV: just the mesons from this region are determined by the short- $r$ and intermediate- $r$ forces, and only the forces from this $r$-region are not known sufficiently well thus being a subject of discussions and hypotheses.

The paper is organized as follows. In Section 2 we recall basic statements of the Bethe-Salpeter equation written in terms of the standard Feynman diagram technique, give the elements of the dispersion relation $N / D$-method, and clarify the interplay of these two methods by using separable vertices. The spectral integral representation of the Bethe-Salpeter equation is also written in this Section for the case of scalar constituents.

In Sect. 3 the $q \bar{q}$ system is considered: the Bethe-Salpeter equations are written for the light-quark mesons which belong to the following $\left(n, M^{2}\right)$-trajectories: $\pi_{J}, \eta_{J}, a_{J}, f_{J}, \rho_{J}, \omega_{J}, h_{J}$, and $b_{J}$.

In Appendices A, B, and C, the necessary auxillary formulae are presented which were used for deriving the equations. In Appendix D we collect equations which are rather cumbersome, these are the Bethe-Salpeter equations for $\omega, \phi, a_{2}$, and $f_{2}$ trajectories. 


\section{SCALAR CONSTITUENTS: DISPERSION RELATION METHOD AND THE BETHE-SALPETER EQUATION FOR COMPOSITE PARTICLES}

In this Section, we compare the Bethe-Salpeter equation for composite particles written with the use of Feynman diagrams with the equation in terms of the dispersion relations with separable vertices. This comparison gives us a guide for the transformation of Bethe-Salpeter equation with separable vertices into the spectral-integral BetheSalpeter equation with arbitrary meson-exchange-type interaction.

To simplify the consideration we deal here with scalar particles as constituents.

\section{A. Bethe-Salpeter equation in the Feynman-diagram technique}

Written in terms of the Feyman diagrams, the nonhomogeneous Bethe-Salpeter equation in the momentum representation reads:

$$
\begin{aligned}
A\left(k_{1}, k_{2} ; k_{1}^{\prime \prime}, k_{2}^{\prime \prime}\right) & =V\left(k_{1}, k_{2} ; k_{1}^{\prime \prime}, k_{2}^{\prime \prime}\right)+\int \frac{d^{4} k_{1}^{\prime} d^{4} k_{2}^{\prime}}{i(2 \pi)^{4}} V\left(k_{1}, k_{2} ; k_{1}^{\prime}, k_{2}^{\prime}\right) \\
& \times \frac{\delta^{4}\left(k_{1}^{\prime}+k_{2}^{\prime}-P\right)}{\left(m^{2}-k_{1}^{\prime 2}-i 0\right)\left(m^{2}-k_{2}^{\prime 2}-i 0\right)} A\left(k_{1}^{\prime}, k_{2}^{\prime} ; k_{1}^{\prime \prime} k_{2}^{\prime \prime}\right) .
\end{aligned}
$$

It is shown in Fig. 1 in the diagram form, and one can see there the notations for particle momenta. In (4) the constituents obey the momentum-conservation constraint:

$$
k_{1}+k_{2}=k_{1}^{\prime}+k_{2}^{\prime}=k_{1}^{\prime \prime}+k_{2}^{\prime \prime}=P,
$$

and $V\left(k_{1}, k_{2} ; k_{1}^{\prime}, k_{2}^{\prime}\right)$ is the irreducible kernel, i.e., the block without two-particle intermediate states (dashed block in Fig. 1).

The scattering amplitude $A\left(k_{1}, k_{2} ; k_{1}^{\prime \prime}, k_{2}^{\prime \prime}\right)$ determined by the Bethe-Salpeter equation (4) is the mass-off-shell amplitude. Even if we set $k_{1}^{2}=k_{1}^{\prime \prime 2}=k_{2}^{2}=k_{2}^{\prime \prime 2}=m^{2}$ in the left-hand side of Eq. (4), the right-hand side contains the amplitude $A\left(k_{1}^{\prime}, k_{2}^{\prime} ; k_{1}^{\prime \prime}, k_{2}^{\prime \prime}\right)$ for $k_{1}^{\prime 2} \neq m^{2}, k_{2}^{\prime 2} \neq m^{2}$.

Let us draw the kernel $V$ as a meson-exchange diagram; then, by iterating Eq. (4), we represent $A\left(k_{1}, k_{2} ; k_{1}^{\prime \prime}, k_{2}^{\prime \prime}\right)$ as an infinite set of ladder diagrams of Fig. 2a. For further investigation it is important to fix intermediate states in the scattering amplitude. The ladder diagrams have two-particle intermediate states which can appear as real states at the c.m. energies squared $s=P^{2}>4 m^{2}$, that corresponds to the cutting of ladder diagrams across the lines related to the constituents, see Fig. $2 \mathrm{~b}$.

Such a two-particle state manifests itself as a singularity of the scattering amplitude at $s=4 \mathrm{~m}^{2}$. However, the amplitude $A\left(k_{1}, k_{2} ; k_{1}^{\prime \prime}, k_{2}^{\prime \prime}\right)$ considered as a function of $s$ has not only this singularity but also an infinite set of singularities which correspond to the ladder-diagram cuts across meson lines associated with the forces: the example of such a cutting is shown in Fig. 2c. The diagrams, which appear after the cut procedure, are the meson-production diagrams, e.g., see Fig. 2d.

So, in the complex $s$-plane the amplitude $A\left(k_{1}, k_{2} ; k_{1}^{\prime \prime}, k_{2}^{\prime \prime}\right)$ has the following singularity:

$$
s=4 m^{2}
$$

which is related to the rescattering process. The other singularities are related to the meson production processes with cuts originating at

$$
s=(2 m+n \mu)^{2}, \quad n=1,2,3, \ldots
$$

The four-point amplitude depends on six variables as follows:

$$
\begin{aligned}
& k_{1}^{2}, k_{2}^{2}, k_{1}^{\prime \prime 2}, k_{2}^{\prime \prime 2}, \\
s= & \left(k_{1}+k_{2}\right)^{2}=\left(k_{1}^{\prime \prime}+k_{2}^{\prime \prime}\right)^{2}, \\
t= & \left(k_{1}-k_{1}^{\prime \prime}\right)^{2}=\left(k_{2}-k_{2}^{\prime \prime}\right)^{2} .
\end{aligned}
$$

The seventh variable, $u=\left(k_{1}-k_{2}^{\prime \prime}\right)^{2}=\left(k_{1}^{\prime \prime}-k_{2}\right)^{2}$, is not independent because of the relation

$$
s+t+u=k_{1}^{2}+k_{2}^{2}+k_{1}^{\prime \prime 2}+k_{2}^{\prime \prime 2} \text {. }
$$


If the interaction creates a bound state, then the infinite set of ladder diagrams should produce the pole singularity in the amplitude. Near the pole, the scattering amplitude is determined by the diagrams of Fig. 3a type that means that in the graphical form the equation for composite system reads as Fig. 3b. In terms of the Feynman integral, it is as follows:

$$
\begin{aligned}
A\left(k_{1}, k_{2} ; P\right) & =\int \frac{d^{4} k_{1}^{\prime} d^{4} k_{2}^{\prime}}{i(2 \pi)^{4}} V\left(k_{1}, k_{2} ; k_{1}^{\prime}, k_{2}^{\prime}\right) \times \\
& \times \frac{\delta^{4}\left(k_{1}^{\prime}+k_{2}^{\prime}-P\right)}{\left(m^{2}-k_{1}^{\prime 2}-i 0\right)\left(m^{2}-k_{2}^{\prime 2}-i 0\right)} A\left(k_{1}^{\prime}, k_{2}^{\prime}, P\right) .
\end{aligned}
$$

The homogeneous Bethe-Salpeter equation (9), like nonhomogeneous one, works upon the mass-off-shell amplitudes; the multimeson production channels in (9) exist, and they are strongly related to the meson-exchange forces.

\section{B. Scattering amplitude in the dispersion relation $N / D$-method}

Let us summarize analytical properties of the discussed scattering amplitudes for two spinless particles (with the mass $m$ ) which interact through the exchange of another spinless particle (with the mass $\mu$ ), Fig. 2a. This amplitude, $A(s, t)$, has $s$ - and $t$-channel singularities. In the $t$-plane, there are singularities at $t=\mu^{2}, 4 \mu^{2}, 9 \mu^{2}$, etc., which correspond to the one- or multi-meson exchanges. In the $s$-plane the amplitude has the singularity at $s=4 m^{2}($ elastic rescattering) and singularities at $s=(2 m+n \mu)^{2}$, where $n=1,2, \ldots$, that corresponds to the production of $n$ mesons with the mass $\mu$. For the bound state with mass $M$, there exists a pole singularity at $s=M^{2}$. If a mass of this bound state $M>2 m$, this is a resonance, and corresponding pole is located on the second sheet of the complex $s$-plane.

The dispersion-relation $N / D$-method deals with partial-wave amplitudes. The $s$-channel partial amplitudes depend on $s$ only. They have all the $s$-channel singularities of $A(s, t)$, namely, the right-hand-side singularities at $s=M^{2}$, $s=4 m^{2}, s=(2 m+\mu)^{2}$, and so on, see Fig. 4 .

Left-hand-side singularities of the partial amplitudes are related to the $t$-channel singularities of $A(s, t)$. The $S$-wave partial amplitude is equal to

$$
A(s)=\int_{-1}^{1} \frac{d z}{2} A(s, t(z))
$$

where $t(z)=-2\left(s / 4-m^{2}\right)(1-z)$ and $z=\cos \theta$. The left-hand singularities correspond to

$$
t(z=-1)=(n \mu)^{2}
$$

and they are located at $s=4 m^{2}-\mu^{2}, s=4 m^{2}-4 \mu^{2}$, and so on.

The $N / D$-method provides us with the possibility to construct the relativistic two-particle scattering amplitude in the region of low and intermediate energies, where multiparticle production processes are not important; this region is shown in Fig. 4 by dashed line. If the threshold singularity at $s=(2 m+\mu)^{2}$ is not strong (one-meson production process is suppressed), the region of partial amplitude under consideration can be expanded up to the next threshold.

The unitarity condition for the partial-wave scattering amplitude (we consider the $S$-wave amplitude as an example) reads:

$$
\operatorname{Im} A(s)=\rho(s)|A(s)|^{2}
$$

Here $\rho(s)$ is the two-particle phase space integrated at fixed $s$ :

$$
\begin{aligned}
\rho(s) & =\int d \Phi_{2}\left(P ; k_{1}^{\prime}, k_{2}^{\prime}\right)=\frac{1}{16 \pi} \sqrt{\frac{s-4 m^{2}}{s}} \\
d \Phi_{2}\left(P ; k_{1}^{\prime}, k_{2}^{\prime}\right) & =\frac{1}{2}(2 \pi)^{4} \delta^{4}\left(P-k_{1}^{\prime}-k_{2}^{\prime}\right) \frac{d^{3} k_{1}^{\prime}}{(2 \pi)^{3} 2 k_{10}^{\prime}} \frac{d^{3} k_{2}^{\prime}}{(2 \pi)^{3} 2 k_{20}^{\prime}} .
\end{aligned}
$$

In the $N / D$-method the amplitude $A(s)$ is represented as

$$
A(s)=\frac{N(s)}{D(s)},
$$


where $N(s)$ has left-hand singularities only, whereas $D(s)$ has the right-hand ones only. So the $N$-function is real in the physical region, $s>4 m^{2}$. The unitarity condition can be rewritten as follows:

$$
\operatorname{Im} D(s)=-\rho(s) N(s) .
$$

The solution of this equation is

$$
D(s)=1-\int_{4 m^{2}}^{\infty} \frac{d s^{\prime}}{\pi} \frac{\rho\left(s^{\prime}\right) N\left(s^{\prime}\right)}{s^{\prime}-s} \equiv 1-B(s) .
$$

In Eq. (16) we suppose that the CDD poles [21] are absent and we normalize $N(s)$ by the condition $D(s) \rightarrow 1$ as $s \rightarrow \infty$.

In principle, Eqs. (14), (16) provide us with a complete description of partial amplitude in the low- $s$ region: the amplitude is determined by the $N$-function being a set of the left-hand singularities which are due to the one-meson exchange $\left(s=4 m^{2}-\mu^{2}\right)$, two-meson exchange $\left(s=4 m^{2}-4 \mu^{2}\right)$, and so on. The right-hand singularities in Eqs. (14), (16) are uncoupled to the left-hand ones, opposite to the Feynman-diagram approach given by (4). It is important for the description of the realistic processes to have the left-hand and right-hand singularities uncoupled: a well-known example provides us with the $p n$-amplitude, with the deuteron quantum numbers, where the production of pions is suppressed (right-hand singularity at $s=\left(2 m+\mu_{\pi}\right)^{2}$ is weak), while the forces related to the pion exchange are significant (left-hand singularity at $s=4 m^{2}-\mu_{\pi}^{2}$ is strong).

\section{C. $N / D$-method and separable interaction}

The $N / D$-method gives us the mass-on-shell partial amplitude, provided the $N$-function is known. However, the $N$-functions have rather intricate properties: they depend on the total number of the $t$ - and $u$-channel exchanges and do not obey the factorization constraints, i.e.,for different reactions the $N$-functions may be independently different. As was stressed above, the spectral integral representation for the Bethe-Salpeter equation, keeping advantages of the dispersion relation method, is free from this problem: it uses $t$ - and $u$ - channel exchanges, with universal interaction blocks.

As the first step in rewriting the Bethe-Salpeter equation in the spectral-integral form, let us consider separable interaction as an example. For this purpose, we rewrite Eqs. (14), (16) introducing the vertex function

$$
g(s)=\sqrt{N(s)} .
$$

Here we assume that $N(s)$ is positive (the cases with negative $N(s)$ or with changing-sign $N(s)$ need the introduction of several vertices). So, the partial wave amplitude $A(s)$ written in terms of the separable vertex $g(s)$ is given by the following series:

$$
A(s)=g(s)\left[1+B(s)+B^{2}(s)+B^{3}(s)+\cdots\right] g(s) .
$$

Its graphic interpretation is shown by Fig. 5 .

This set of diagrams can be rewritten in the form of the Bethe-Salpeter equation:

$$
A(s)=g^{2}(s)+g(s) \int_{4 m^{2}}^{\infty} \frac{d s^{\prime}}{\pi} g\left(s^{\prime}\right) \frac{\rho\left(s^{\prime}\right)}{s^{\prime}-s} A\left(s^{\prime}, s\right),
$$

where $A\left(s^{\prime}, s\right)$ is the energy-off-shell amplitude which enters the intermediate state of the diagrams of Fig. 5; one has for the energy-on-shell amplitude $A(s, s)=A(s)$. The interaction block is written as follows:

$$
V\left(s^{\prime}, s\right)=g\left(s^{\prime}\right) g(s)
$$

Therefore, the Bethe-Salpeter equation is to be applied for the amplitude $A\left(s, s^{\prime}\right)$; it reads:

$$
A\left(s^{\prime}, s\right)=g\left(s^{\prime}\right) g(s)+g\left(s^{\prime}\right) \int_{4 m^{2}}^{\infty} \frac{d s^{\prime \prime}}{\pi} g\left(s^{\prime \prime}\right) \frac{\rho\left(s^{\prime \prime}\right)}{s^{\prime \prime}-s} A\left(s^{\prime \prime}, s\right) .
$$


If the bound state exists, the amplitude contains a pole singularity at $s=M^{2}$. Considering Eq. (21) near the pole and neglecting the non-pole terms, we have the following equation for the bound state vertex:

$$
G\left(s^{\prime}, M^{2}\right)=g\left(s^{\prime}\right) \int_{4 m^{2}}^{\infty} \frac{d s^{\prime \prime}}{\pi} g\left(s^{\prime \prime}\right) \frac{\rho\left(s^{\prime \prime}\right)}{s^{\prime \prime}-M^{2}} G\left(s^{\prime \prime}, M^{2}\right),
$$

where

$$
G\left(s^{\prime}, M^{2}\right)=\left[\frac{A\left(s^{\prime}, s\right)\left(M^{2}-s\right)}{G(s)}\right]_{s \rightarrow M^{2}} .
$$

The Bethe-Salpeter equation (22) gives us a guide for the consideration of general case, when the interaction is of the meson-exchange type. But before we need to consider in more detail the representation of the loop diagram.

\section{Loop diagram}

The loop diagram $B(s)$ plays the decisive role for the two-meson amplitude, so let us compare in details the dispersion and Feynman integral expressions for $B(s)$.

Namely, the Feynman expression for $B_{F}(s)$, with a special choice of separable interaction $G\left(4 k_{\perp}^{2}+4 m^{2}\right)$, is proved to be equal to the dispersion integral representation, where the four-vector $k_{\perp}$ is defined as follows:

$$
2 k_{\perp}=k_{1}-k_{2}-\frac{k_{1}^{2}-k_{2}^{2}}{P^{2}}\left(k_{1}+k_{2}\right) .
$$

In this Section, we use the total-momentum vector $P=k_{1}+k_{2}$, so it is convenient to write here $P^{2}$ but not $s$.

The Feynman expression for the loop diagram reads:

$$
B_{F}\left(P^{2}\right)=\frac{1}{(2 \pi)^{4} i} \int \frac{d^{4} k_{2} G^{2}\left(4\left(P k_{2}\right)^{2} / P^{2}-4 k_{2}^{2}+4 m^{2}\right)}{\left(m^{2}-k_{2}^{2}-i 0\right)\left(m^{2}-\left(P-k_{2}\right)^{2}-i 0\right)} .
$$

Since it is more convenient to treat composite system with light-cone variables, they are hereafter:

$$
k_{-}=\frac{1}{\sqrt{2}}\left(k_{20}-k_{2 z}\right) ; \quad k_{+}=\frac{1}{\sqrt{2}}\left(k_{20}+k_{2 z}\right) ; \quad \vec{k}_{2 \perp}=\vec{k}_{T} .
$$

We choose the reference frame, where $P_{T}=0$. Then

$$
P k_{2}=P_{+} k_{-}+P_{-} k_{+},
$$

and Eq. (25) takes the form

$$
\begin{gathered}
B_{F}\left(P^{2}\right)=\frac{1}{(2 \pi)^{4} i} \times \\
\times \int \frac{d k_{+} d k_{-} d^{2} k_{T}}{\left(2 k_{+} k_{-}-m_{T}^{2}+i 0\right)\left(P^{2}-2\left(P_{+} k_{-}+P_{-} k_{+}\right)+2 k_{+} k_{-}-m_{T}^{2}+i 0\right)},
\end{gathered}
$$

where $m_{T}^{2}=m^{2}+k_{T}^{2}$. It should be mentioned,that if $G \equiv 1$, one can perform the integration over $k_{-}$right now, closing the integration contour around the pole

$$
k_{-}=\frac{m_{T}^{2}-i 0}{2 k_{+}},
$$

and we obtain the standard dispersion representation for the Feynman loop graph $\left(x=k_{+} / P_{+}\right)$: 


$$
\begin{aligned}
\frac{1}{(2 \pi)^{4} i} \int d^{2} k_{T} \int_{0}^{1} d x \frac{(-2 \pi i)}{2 x\left(P^{2}-m_{T}^{2} / x-P^{2} x+i 0\right)}=\int \frac{d s}{\pi\left(s-P^{2}-i 0\right)} \times \\
\times \quad \int \frac{d x d k_{T}^{2}}{x(1-x)} \frac{\delta\left(s-m_{T}^{2} /[x(1-x)]\right)}{16 \pi}=\int_{4 m^{2}}^{\infty} \frac{d s \cdot \rho(s)}{\pi\left(s-P^{2}-i 0\right)} .
\end{aligned}
$$

The dispersion integral (30) is divergent at $s \rightarrow \infty$ due to $G=1$, and it is the function $G$ which makes $B_{F}$ convergent in Eq. (25). Convergence of the integral (30) can be restored by the subtraction procedure.

For $G \neq 1$, some additional steps are needed to obtain the dispersion representation, namely, we introduce new variables $\xi_{+}$and $\xi_{-}$:

$$
\begin{aligned}
& P_{+} k_{-}+P_{-} k_{+}=\sqrt{P^{2}} \xi_{+} \\
& P_{+} k_{-}-P_{-} k_{+}=\sqrt{P^{2}} \xi_{-} .
\end{aligned}
$$

With these variables, Eq. (25) takes the form

$$
\begin{aligned}
B_{F}\left(P^{2}\right) & =\frac{1}{(2 \pi)^{4} i} \times \\
& \times \int \frac{G^{2}\left(4\left(\xi_{-}^{2}+m_{T}^{2}\right)\right) d \xi_{+} d \xi_{-} d^{2} k_{T}}{\left(\xi_{+}^{2}-\xi_{-}^{2}-m_{T}^{2}+i 0\right)\left(P^{2}-2 \sqrt{P^{2}} \xi_{+}+\xi_{+}^{2}-\xi_{-}^{2}-m_{T}^{2}+i 0\right)}= \\
& =\int d k_{T}^{2} \int_{0}^{\infty} 2 d \xi_{-} \pi G^{2}\left(4\left(\xi_{-}^{2}+m_{T}^{2}\right)\right) \times \\
& \times \int_{-\infty}^{\infty} \frac{d \xi_{+}}{\left.\left[\xi_{+}^{2}-\left(\xi_{-}^{2}+m_{T}^{2}\right)+i 0\right]\left[\left(\xi_{+}-\sqrt{P^{2}}\right)^{2}-\left(\xi_{-}^{2}+m_{T}^{2}\right)+i 0\right)\right]} .
\end{aligned}
$$

The integration over $\xi_{+}$is performed by closing the integration contour in the upper half-plane, so the two poles, $\xi_{+}=-\sqrt{\xi_{-}^{2}+m_{T}^{2}}+i 0$ and $\xi_{+}=\sqrt{P^{2}}-\sqrt{\xi_{-}^{2}+m_{T}^{2}}+i 0$, contribute. The introduction of a new variable $s=4\left(\xi_{-}^{2}+m_{T}^{2}\right)$ yields

$$
B_{F}\left(P^{2}\right)=\int_{4 m^{2}}^{\infty} \frac{d s G^{2}(s)}{\pi\left(s-P^{2}\right)} \frac{1}{16 \pi} \sqrt{1-\frac{4 m^{2}}{s}},
$$

that is the dispersion representation of Eq. (16).

So the hypothesis of the separable interaction gives us an opportunity to solve the Bethe-Salpeter equation easily. Within this hypothesis we can use different techniques: either Feynman integration, or spectral-integral representation, or light-cone variables.

\section{E. Spectral-integral representation and interaction forces}

The introduction of a separable interaction is not the only way to make the Bethe-Salpeter equation easily solvable. The main point in handling the Bethe-Salpeter equation is to control the right-hand side singularities, especially those related to multimeson production, at $s=(2 m+\mu)^{2}, s=(2 m+2 \mu)^{2}, \ldots$, and it is the spectral integration technique which enables us to control the multimeson production processes.

The spectral integral representation is based on the following corner stones:

(i) constituent particles in the intermediate states are mass-on-shell $\left(k_{1}^{\prime 2}=m^{2}\right.$ and $k_{2}^{\prime 2}=m^{2}$ in Fig. 1); (ii) there is no energy conservation in the interaction processes $\left(s \neq s^{\prime} \neq s^{\prime \prime}\right.$ in Fig. 5$)$.

Based on these statements we consider potential interaction, or the particle-exchange interaction, by using the spectral-integral diagrams. Consider as an example the interaction associated with the $t$-channel exchange by a meson with the mass $\mu$ :

$$
V\left(k_{1}, k_{1}^{\prime}\right)=\frac{g^{2}}{\mu^{2}-t}, \quad t=\left(k_{1}-k_{1}^{\prime}\right)^{2}
$$


In the c.m. system, which is the most convenient for the consideration, one has for the four-momenta of the constituent particles:

$$
\begin{array}{r}
k_{1}=\left(k_{0}, \vec{k}\right)=\left(\frac{\sqrt{s}}{2}, \vec{n} \sqrt{\frac{s}{4}-m^{2}}\right), \\
k_{1}^{\prime}=\left(k_{0}^{\prime}, \vec{k}^{\prime}\right)=\left(\frac{\sqrt{s^{\prime}}}{2}, \vec{n}^{\prime} \sqrt{\frac{s^{\prime}}{4}-m^{2}}\right),
\end{array}
$$

where $\vec{n}^{2}=\vec{n}^{\prime 2}=1$. The interaction block $V\left(k_{1}, k_{1}^{\prime}\right)$ can be expanded in a series with respect to $z=\left(\vec{n} \vec{n}^{\prime}\right)$. In this way, we may obtain the interaction for different partial waves. For example, the interaction in the wave with the angular momentum $L=0$ is equal to:

$$
V_{0}\left(s, s^{\prime}\right)=\int_{-1}^{1} \frac{d z}{2} V\left(k_{1}, k_{1}^{\prime}\right)
$$

Actually, Eqs. (34),(35),(36) allow us to generalize the procedure with the separable interaction considered above. Indeed, expanding (36) in a series with respect to orthogonal functions, one has

$$
V_{0}\left(s, s^{\prime}\right)=\sum_{n} g_{n}(s) g_{n}\left(s^{\prime}\right),
$$

that is a separable interaction in a generalized form, supposing the choice of the functions allows one to use a restricted number of terms in (37). Separable interaction taken in such a form was used in [3,4] for the description of nucleon-nucleon interactions by considering the deuteron within dispersion-relation technique.

\section{F. Spectral-integral representation of the Bethe- Salpeter equation for composite system}

First, we consider the case of $L=0$ for scalar constituents with equal masses, though not identical. The bound system is treated as a composite system of these constituents. Furthermore the case $L \neq 0$ is considered in detail.

\section{Bethe-Salpeter equation for vertex function with $L=0$}

The equation for the vertex composite system $\rightarrow$ constituents, shown graphically in Fig. 3b, reads:

$$
G(s)=\int_{4 m^{2}}^{\infty} \frac{d s^{\prime}}{\pi} \int d \Phi_{2}\left(P^{\prime} ; k_{1}^{\prime}, k_{2}^{\prime}\right) V\left(k_{1}, k_{2} ; k_{1}^{\prime}, k_{2}^{\prime}\right) \frac{G\left(s^{\prime}\right)}{s^{\prime}-M^{2}-i 0},
$$

where the phase space is determined by Eq. (13). Scalar constituents are supposed to be not identical, so we do not write additional identity factor $1 / 2$ in the phase space.

The equation (38) written in the spectral-representation form deals with the energy off-shell states $s^{\prime}=\left(k_{1}^{\prime}+k_{2}^{\prime}\right)^{2} \neq$ $M^{2}, s=\left(k_{1}+k_{2}\right)^{2} \neq M^{2}$ and $s \neq s^{\prime}$; the constituents are mass-on-shell, $k_{1}^{\prime 2}=m^{2}$ and $k_{2}^{\prime 2}=m^{2}$. We can use alternative expression for the phase space:

$$
d \Phi_{2}\left(P^{\prime} ; k_{1}^{\prime}, k_{2}^{\prime}\right)=\rho\left(s^{\prime}\right) \frac{d z}{2} \equiv d \Phi\left(k^{\prime}\right), \quad z=\frac{\left(k k^{\prime}\right)}{\sqrt{k^{2}} \sqrt{k^{\prime 2}}},
$$

where $k=\left(k_{1}-k_{2}\right) / 2$ and $k^{\prime}=\left(k_{1}^{\prime}-k_{2}^{\prime}\right) / 2$. Then

$$
G(s)=\int_{4 m^{2}}^{\infty} \frac{d s^{\prime}}{\pi} \int d \Phi\left(k^{\prime}\right) V\left(s, s^{\prime},\left(k k^{\prime}\right)\right) \frac{G\left(s^{\prime}\right)}{s^{\prime}-M^{2}-i 0} .
$$

In the c.m. system $\left(k k^{\prime}\right)=-\left(\mathbf{k k}^{\prime}\right)$ and $\sqrt{k^{2}}=\sqrt{-\mathbf{k}^{2}}=i|\mathbf{k}|$ and $\sqrt{k^{\prime 2}}=\sqrt{-\mathbf{k}^{\prime 2}}=i\left|\mathbf{k}^{\prime}\right|$ so $z=\left(\mathbf{k} \mathbf{k}^{\prime}\right) /\left(|\mathbf{k}|\left|\mathbf{k}^{\prime}\right|\right)$. The phase space and spectral integrations can be written as follows: 


$$
\int_{4 m^{2}}^{\infty} \frac{d s^{\prime}}{\pi} \int d \Phi_{2}\left(P^{\prime} ; k_{1}^{\prime}, k_{2}^{\prime}\right)=\int \frac{d \mathbf{k}^{\prime}}{(2 \pi)^{3} k_{0}^{\prime}}
$$

where $k_{0}^{\prime}=\sqrt{m^{2}+\mathbf{k}^{\prime 2}}$. In the c.m. system Eq. (38) reads

$$
G(s)=\int \frac{d \mathbf{k}^{\prime}}{(2 \pi)^{3} k_{0}^{\prime}} V\left(s, s^{\prime},-\left(\mathbf{k k}^{\prime}\right)\right) \frac{G\left(s^{\prime}\right)}{s^{\prime}-M^{2}-i 0} .
$$

\section{Bethe-Salpeter equation for the $(L=0)$-wave function}

Now consider the wave function of composite system,

$$
\psi(s)=\frac{G(s)}{s-M^{2}} .
$$

To this aim, the identity transformation upon the equation (42) should be done as follows:

$$
\left(s-M^{2}\right) \frac{G(s)}{s-M^{2}}=\int_{4 m^{2}}^{\infty} \frac{d s^{\prime}}{\pi} \int d \Phi\left(k^{\prime}\right) V\left(s, s^{\prime},\left(k k^{\prime}\right)\right) \frac{G\left(s^{\prime}\right)}{s^{\prime}-M^{2}} .
$$

Using the wave functions, the equation (44) can be written as follows:

$$
\left(s-M^{2}\right) \psi(s)=\int_{4 m^{2}}^{\infty} \frac{d s^{\prime}}{\pi} \int d \Phi_{2}\left(k^{\prime}\right) V\left(s, s^{\prime},\left(k k^{\prime}\right)\right) \psi\left(s^{\prime}\right) .
$$

Finally, using $\mathbf{k}^{\prime 2}$ and $\mathbf{k}^{2}$ instead of $s^{\prime}$ and $s$,

$$
\psi(s) \rightarrow \psi\left(\mathbf{k}^{2}\right)
$$

we have:

$$
\left(4 \mathbf{k}^{2}+4 m^{2}-M^{2}\right) \psi\left(\mathbf{k}^{2}\right)=\int \frac{d \mathbf{k}^{\prime}}{(2 \pi)^{3} k_{0}^{\prime}} V\left(s, s^{\prime},-\left(\mathbf{k k}^{\prime}\right)\right) \psi\left(\mathbf{k}^{\prime 2}\right) .
$$

This is a basic equation for the set of states with $L=0$. The set is formed by the levels with different radial excitations $n=1,2,3, \ldots$, and relevant wave functions are as follows:

$$
\psi_{1}\left(\mathbf{k}^{2}\right), \psi_{2}\left(\mathbf{k}^{2}\right), \psi_{3}\left(\mathbf{k}^{2}\right), \ldots
$$

The wave functions are normalized and orthogonal to each other. The normalization/orthogonality condition reads:

$$
\int \frac{d \mathbf{k}}{(2 \pi)^{3} k_{0}} \psi_{n}\left(\mathbf{k}^{2}\right) \psi_{n^{\prime}}\left(\mathbf{k}^{2}\right)=\delta_{n n^{\prime}}
$$

Here $\delta_{n n^{\prime}}$ is the Kronecker symbol. The equation (47) is due to the consideration of the charge form factors of composite systems with the gauge-invariance requirement imposed, see for detail [13]. This normalization/ orthogonality condition looks as in quantum mechanics.

Therefore, the Bethe-Salpeter equation for the $S$-wave mesons reads:

$$
4\left(\mathbf{k}^{2}+m^{2}\right) \psi_{n}\left(\mathbf{k}^{2}\right)-\int_{0}^{\infty} \frac{d \mathbf{k}^{\prime 2}}{\pi} V_{0}\left(\mathbf{k}^{2}, \mathbf{k}^{\prime 2}\right) \phi\left(\mathbf{k}^{\prime 2}\right) \psi_{n}\left(\mathbf{k}^{\prime 2}\right)=M_{n}^{2} \psi_{n}\left(\mathbf{k}^{2}\right),
$$

where 


$$
\phi\left(\mathbf{k}^{\prime 2}\right)=\frac{1}{4 \pi} \frac{\left|\mathbf{k}^{\prime}\right|}{k_{0}^{\prime}} .
$$

The $\psi_{n}\left(\mathbf{k}^{2}\right)$ presents a full set of wave functions which are orthogonal and normalized:

$$
\int_{0}^{\infty} \frac{d \mathbf{k}^{2}}{\pi} \psi_{a}\left(\mathbf{k}^{2}\right) \phi\left(\mathbf{k}^{2}\right) \psi_{b}\left(\mathbf{k}^{2}\right)=\delta_{a b} .
$$

The function $V_{0}\left(\mathbf{k}^{2}, \mathbf{k}^{\prime 2}\right)$ is the projection of potential $V\left(s, s^{\prime},\left(k k^{\prime}\right)\right)$ on the $S$-wave:

$$
V_{0}\left(\mathbf{k}^{2}, \mathbf{k}^{\prime 2}\right)=\int \frac{d \Omega_{\mathbf{k}}}{4 \pi} \int \frac{d \Omega_{\mathbf{k}^{\prime}}}{4 \pi} V\left(s, s^{\prime},-\left(\mathbf{k k}^{\prime}\right)\right) .
$$

Let us expand $V_{0}\left(\mathbf{k}^{2}, \mathbf{k}^{\prime 2}\right)$ with respect to full set of wave functions:

$$
V_{0}\left(\mathbf{k}^{2}, \mathbf{k}^{\prime 2}\right)=\sum_{a, b} \psi_{a}\left(\mathbf{k}^{2}\right) v_{a b}^{(0)} \psi_{b}\left(\mathbf{k}^{\prime 2}\right)
$$

where numerical coefficients $v_{a b}^{(0)}$ are defined by the inverse transformation as follows:

$$
v_{a b}^{(0)}=\int_{0}^{\infty} \frac{d \mathbf{k}^{2}}{\pi} \frac{d \mathbf{k}^{\prime 2}}{\pi} \psi_{a}\left(\mathbf{k}^{2}\right) \phi\left(\mathbf{k}^{2}\right) V_{0}\left(\mathbf{k}^{2}, \mathbf{k}^{\prime 2}\right) \phi\left(\mathbf{k}^{\prime 2}\right) \psi_{b}\left(\mathbf{k}^{\prime 2}\right) .
$$

Taking account of a series (52), the equation (48) is rewritten as follows:

$$
4\left(\mathbf{k}^{2}+m^{2}\right) \psi_{n}\left(\mathbf{k}^{2}\right)-\sum_{a} \psi_{a}\left(\mathbf{k}^{2}\right) v_{a n}^{(0)}=M_{n}^{2} \psi_{n}\left(\mathbf{k}^{2}\right)
$$

Such a transformation should be carried out upon the kinetic-energy term, it is also expanded in a series with respect to a full set of wave functions:

$$
4\left(\mathbf{k}^{2}+m^{2}\right) \psi_{n}\left(\mathbf{k}^{2}\right)=\sum_{a} K_{n a} \psi_{a}\left(\mathbf{k}^{2}\right)
$$

where

$$
K_{n a}=\int_{0}^{\infty} \frac{d \mathbf{k}^{2}}{\pi} \psi_{a}\left(\mathbf{k}^{2}\right) \phi\left(\mathbf{k}^{2}\right) 4\left(\mathbf{k}^{2}+m^{2}\right) \psi_{n}\left(\mathbf{k}^{2}\right) .
$$

Finally, the Bethe-Salpeter equation takes the form:

$$
\sum_{a} K_{n a} \psi_{a}\left(\mathbf{k}^{2}\right)-\sum_{a} v_{n a}^{(0)} \psi_{a}\left(\mathbf{k}^{2}\right)=M_{n}^{2} \psi_{n}\left(\mathbf{k}^{2}\right) .
$$

We take into account that $v_{n a}^{(0)}=v_{a n}^{(0)}$.

The equation (57) is a standard homogeneous equation:

$$
\sum_{a} s_{n a} \psi_{a}\left(\mathbf{k}^{2}\right)=M_{n}^{2} \psi_{n}\left(\mathbf{k}^{2}\right)
$$

with $s_{n a}=K_{n a}-v_{n a}^{(0)}$. The values $M^{2}$ are defined as zeros of the determinant

$$
\operatorname{det}\left|\hat{s}-M^{2} I\right|=0,
$$

where $I$ is the unity matrix. 
3. The Bethe-Salpeter equation for the states with arbitary angular momentum $L$

For the wave function with arbitrary angular momentum $\psi_{(n) \mu_{1}, \ldots, \mu_{L}}^{(L)}(s)$, we use the following ansatz:

$$
\psi_{(n) \mu_{1}, \ldots, \mu_{L}}^{(L)}(s)=X_{\mu_{1}, \ldots, \mu_{L}}^{(L)}(k) \psi_{n}^{(L)}(s) .
$$

The momentum operator $X_{\mu_{1}, \ldots, \mu_{L}}^{(L)}(k)$ was introduced in [9], we remind its features in Appendix A.

The Bethe-Salpeter equation for the $(L, n)$-state, presented in the form similar to (48), reads:

$$
\begin{gathered}
4\left(\mathbf{k}^{2}+m^{2}\right) X_{\mu_{1}, \ldots, \mu_{L}}^{(L)}(k) \psi_{n}^{(L)}\left(\mathbf{k}^{2}\right)-X_{\mu_{1}, \ldots, \mu_{L}}^{(L)}(k) \times \\
\times \int_{0}^{\infty} \frac{d \mathbf{k}^{\prime 2}}{\pi} V_{L}\left(s, s^{\prime}\right) X_{L}^{2}\left(k^{\prime 2}\right) \phi\left(\mathbf{k}^{\prime 2}\right) \psi_{n}^{(L)}\left(\mathbf{k}^{\prime 2}\right)=M_{n}^{2} X_{\mu_{1}, \ldots, \mu_{L}}^{(L)}(k) \psi_{n}^{(L)}\left(\mathbf{k}^{2}\right),
\end{gathered}
$$

where

$$
\begin{gathered}
X_{L}^{2}\left(k^{\prime 2}\right)=\int \frac{d \Omega_{\mathbf{k}^{\prime}}}{4 \pi}\left(X_{\nu_{1}, \ldots, \nu_{L}}^{(L)}\left(k^{\prime}\right)\right)^{2}=\alpha(L)\left(k^{\prime 2}\right)^{L}=\alpha(L)\left(-\mathbf{k}^{\prime 2}\right)^{L}, \\
\alpha(L)=\frac{(2 L-1) ! !}{L !}, \alpha(0)=1 .
\end{gathered}
$$

The potential is expanded in a series with respect to the product of operators $X_{\mu_{1}, \ldots, \mu_{L}}^{(L)}(k) X_{\mu_{1}, \ldots, \mu_{L}}^{(L)}\left(k^{\prime}\right)$, that is,

$$
\begin{gathered}
V\left(s, s^{\prime},\left(k k^{\prime}\right)\right)=\sum_{L, \mu_{1} \ldots \mu_{L}} X_{\mu_{1}, \ldots, \mu_{L}}^{(L)}(k) V_{L}\left(s, s^{\prime}\right) X_{\mu_{1}, \ldots, \mu_{L}}^{(L)}\left(k^{\prime}\right), \\
X_{L}^{2}\left(k^{2}\right) V_{L}\left(s, s^{\prime}\right) X_{L}^{2}\left(k^{\prime 2}\right)= \\
=\int \frac{d \Omega_{\mathbf{k}}}{4 \pi} \frac{d \Omega_{\mathbf{k}^{\prime}}}{4 \pi} X_{\nu_{1}, \ldots, \nu_{L}}^{(L)}(k) V\left(s, s^{\prime},\left(k k^{\prime}\right)\right) X_{\nu_{1}, \ldots, \nu_{L}}^{(L)}\left(k^{\prime}\right) .
\end{gathered}
$$

Therefore,formula (61) reads as follows:

$$
\begin{gathered}
4\left(\mathbf{k}^{2}+m^{2}\right) \psi_{n}^{(L)}\left(\mathbf{k}^{2}\right)-\int_{0}^{\infty} \frac{d \mathbf{k}^{\prime 2}}{\pi} V_{L}\left(s, s^{\prime}\right) \alpha(L)\left(-\mathbf{k}^{\prime 2}\right)^{L} \phi\left(\mathbf{k}^{\prime 2}\right) \psi_{n}^{(L)}\left(\mathbf{k}^{\prime 2}\right)= \\
=M_{n}^{2} \psi_{n}^{(L)}\left(\mathbf{k}^{2}\right) .
\end{gathered}
$$

As compared to (48) this equation contains additional factor $X_{L}^{2}\left(k^{\prime 2}\right)$; still, the same factor is in the normalization condition, so it would be reasonable to insert it into the phase space. Finally, we have:

$$
4\left(\mathbf{k}^{2}+m^{2}\right) \psi_{n}^{(L)}\left(\mathbf{k}^{2}\right)-\int_{0}^{\infty} \frac{d \mathbf{k}^{\prime 2}}{\pi} \widetilde{V}_{L}\left(s, s^{\prime}\right) \phi_{L}\left(\mathbf{k}^{\prime 2}\right) \psi_{n}^{(L)}\left(\mathbf{k}^{\prime 2}\right)=M_{n}^{2} \psi_{n}^{(L)}\left(\mathbf{k}^{2}\right),
$$

where

$$
\phi_{L}\left(\mathbf{k}^{\prime 2}\right)=\alpha(L)\left(\mathbf{k}^{\prime 2}\right)^{L} \phi\left(\mathbf{k}^{\prime 2}\right), \quad \widetilde{V}_{L}\left(s, s^{\prime}\right)=(-1)^{L} V_{L}\left(s, s^{\prime}\right) .
$$

The normalization condition for a set of wave functions with orbital momentum $L$ reads:

$$
\int_{0}^{\infty} \frac{d \mathbf{k}^{2}}{\pi} \psi_{a}^{(L)}\left(\mathbf{k}^{2}\right) \phi_{L}\left(\mathbf{k}^{2}\right) \psi_{b}^{(L)}\left(\mathbf{k}^{2}\right)=\delta_{a b}
$$


One can see that it is similar to the case of $L=0$, the only difference consists in the redefinition of the phase space $\phi \rightarrow \phi_{L}$. The Bethe-Salpeter equation reads:

$$
\sum_{a} s_{n a}^{(L)} \psi_{a}^{(L)}\left(\mathbf{k}^{2}\right)=M_{n, L}^{2} \psi_{n}^{(L)}\left(\mathbf{k}^{2}\right),
$$

with

$$
\begin{aligned}
s_{n a}^{(L)} & =K_{n a}^{(L)}-v_{n a}^{(L)}, \\
v_{a b}^{(L)} & =\int_{0}^{\infty} \frac{d \mathbf{k}^{2}}{\pi} \frac{d \mathbf{k}^{\prime 2}}{\pi} \psi_{a}^{(L)}\left(\mathbf{k}^{2}\right) \phi_{L}\left(\mathbf{k}^{2}\right) \widetilde{V}_{L}\left(s, s^{\prime}\right) \phi_{L}\left(\mathbf{k}^{\prime 2}\right) \psi_{b}^{(L)}\left(\mathbf{k}^{\prime 2}\right), \\
K_{n a}^{(L)} & =\int_{0}^{\infty} \frac{d \mathbf{k}^{2}}{\pi} \psi_{a}^{(L)}\left(\mathbf{k}^{2}\right) \phi_{L}(\mathbf{k}) 4\left(\mathbf{k}^{2}+m^{2}\right) \psi_{n}^{(L)}\left(\mathbf{k}^{2}\right) .
\end{aligned}
$$

Using radial-excitation levels one can reconstruct the potential in the $L$-wave, then to reconstruct, with the help of (64), the $t$-dependent potential.

\section{QUARK-ANTIQUARK COMPOSITE SYSTEMS}

For the $q \bar{q}$ system, the Bethe-Salpeter equation for the wave function with the total momentum $J$, angular momentum $L=|\vec{J}-\vec{S}|$ and quark-antiquark spin $S$ can be conventionally written as follows:

$$
\left(s-M^{2}\right) \widehat{\Psi}_{(n) \mu_{1} \cdots \mu_{J}}^{(S, L, J)}(k)=\int \frac{d^{3} k^{\prime}}{(2 \pi)^{3} k_{0}^{\prime}} \widehat{V}\left(s, s^{\prime},\left(k k^{\prime}\right)\right) \widehat{\Psi}_{(n) \mu_{1} \cdots \mu_{J}}^{(S, L, J)}\left(k^{\prime}\right),
$$

where

$$
k=\frac{1}{2}\left(k_{1}-k_{2}\right), \quad s=\left(k_{1}+k_{2}\right)^{2}, \quad k^{\prime}=\frac{1}{2}\left(k_{1}^{\prime}-k_{2}^{\prime}\right), \quad s^{\prime}=\left(k_{1}^{\prime}+k_{2}^{\prime}\right)^{2} .
$$

The wave-function operator with the fixed quantum numbers is presented as

$$
\widehat{\Psi}_{(n) \mu_{1} \cdots \mu_{J}}^{(S, L, J)}(k)=\widehat{Q}_{\mu_{1} \cdots \mu_{J}}^{(S, L, J)}(k) \psi_{n}^{(S, L, J)}\left(k^{2}\right),
$$

where $\widehat{Q}$ is the moment operator for the $q \bar{q}$ system.

The potential operator can be decomposed as follows:

$$
\widehat{V}\left(s, s^{\prime},\left(k k^{\prime}\right)\right)=\sum_{I} V_{I}^{(0)}\left(s, s^{\prime},\left(k k^{\prime}\right)\right) \widehat{O}_{I} \otimes \widehat{O}_{I},
$$

where $I=S, V, T, A, P$ is a full set of Dirac matrices in the $t$-channel:

$$
\widehat{O}_{I}=\mathrm{I}, \gamma_{\mu}, i \sigma_{\mu \nu}, i \gamma_{\mu} \gamma_{5}, \gamma_{5}
$$

The potential operator $\widehat{V}\left(s, s^{\prime},\left(k k^{\prime}\right)\right)$ can be decomposed in the $s$ channel by using the Fierz transformation:

$$
\widehat{V}\left(s, s^{\prime},\left(k k^{\prime}\right)\right)=\sum_{I} \sum_{c} \widehat{V}_{I}^{(0)}\left(s, s^{\prime},\left(k k^{\prime}\right)\right) C_{I c}\left(\widehat{O}_{c} \otimes \widehat{O}_{c}\right),
$$

where $C_{I c}$ are coefficients of the Fierz matrix:

$$
C_{I c}=\left(\begin{array}{ccccc}
\frac{1}{4} & \frac{1}{4} & \frac{1}{8} & \frac{1}{4} & \frac{1}{4} \\
1 & -\frac{1}{2} & 0 & \frac{1}{2} & -1 \\
3 & 0 & -\frac{1}{2} & 0 & 3 \\
1 & \frac{1}{2} & 0 & -\frac{1}{2} & -1 \\
\frac{1}{4} & -\frac{1}{4} & \frac{1}{8} & -\frac{1}{4} & \frac{1}{4}
\end{array}\right) .
$$


Denoting

$$
V_{c}\left(s, s^{\prime},\left(k k^{\prime}\right)\right)=\sum_{I} \widehat{V}_{I}^{(0)}\left(s, s^{\prime},\left(k k^{\prime}\right)\right) C_{I c},
$$

we have

$$
\begin{gathered}
\widehat{V}\left(s, s^{\prime},\left(k k^{\prime}\right)\right)=\sum_{c}\left(\widehat{O}_{c} \otimes \widehat{O}_{c}\right) V_{c}\left(s, s^{\prime},\left(k k^{\prime}\right)\right)= \\
=(\mathrm{I} \otimes \mathrm{I}) V_{S}\left(s, s^{\prime},\left(k k^{\prime}\right)\right)+\left(\gamma_{\mu} \otimes \gamma_{\mu}\right) V_{V}\left(s, s^{\prime},\left(k k^{\prime}\right)\right)+\left(i \sigma_{\mu \nu} \otimes i \sigma_{\mu \nu}\right) \times \\
\times V_{T}\left(s, s^{\prime},\left(k k^{\prime}\right)\right)+\left(i \gamma_{\mu} \gamma_{5} \otimes i \gamma_{\mu} \gamma_{5}\right) V_{A}\left(s, s^{\prime},\left(k k^{\prime}\right)\right)+\left(\gamma_{5} \otimes \gamma_{5}\right) V_{P}\left(s, s^{\prime},\left(k k^{\prime}\right)\right) .
\end{gathered}
$$

Let us multiply Eq. (71) by the operator $\widehat{Q}_{\mu_{1} \ldots \mu_{J}}^{(S, L, J)}(k)$ and convolute over the spin-momentum indeces. After redefinition $\widehat{V}\left(s, s^{\prime},\left(k k^{\prime}\right)\right) \rightarrow\left(\hat{k}_{1}^{\prime}+m^{\prime}\right) \widehat{V}\left(s, s^{\prime},\left(k k^{\prime}\right)\right)\left(-\hat{k}_{2}^{\prime}+m^{\prime}\right)$ one has:

$$
\begin{aligned}
& \left(s-M^{2}\right) S p\left[\widehat{\Psi}_{(n) \mu_{1} \ldots \mu_{J}}^{(S, L, J)}(k)\left(\widehat{k}_{1}+m\right) \widehat{Q}_{\mu_{1} \ldots \mu_{J}}^{(S, L, J)}(k)\left(-\widehat{k}_{2}+m\right)\right]= \\
& =\sum_{c} S p\left[\widehat{O}_{c}\left(\widehat{k}_{1}+m\right) \widehat{Q}_{\mu_{1} \ldots \mu_{J}}^{(S, L, J)}(k)\left(-\widehat{k}_{2}+m\right)\right] \int \frac{d^{3} k^{\prime}}{(2 \pi)^{3} k_{0}^{\prime}} V_{c}\left(s, s^{\prime},\left(k k^{\prime}\right)\right) \times \\
& \times S p\left[\left(\widehat{k}_{1}^{\prime}+m^{\prime}\right) \widehat{O}_{c}\left(-\widehat{k}_{2}^{\prime}+m^{\prime}\right) \widehat{\Psi}_{(n) \mu_{1} \ldots \mu_{J}}^{(S, L, J)}\left(k^{\prime}\right)\right] .
\end{aligned}
$$

Here we define $m^{\prime}$ as ${k^{\prime}}_{1}^{2}={k^{\prime}}_{2}^{2}={m^{\prime}}^{2}$. We have four states with the $q \bar{q}$ spins $S=0$ and $S=1$ :

1) $S=0 ; L=J$,

2) $S=1 ; L=J+1, J, J-1$.

These states are constructed from the operators [9] as follows:

$$
\begin{aligned}
& \widehat{Q}_{\mu_{1} \ldots \mu_{J}}^{(0, J, J)}(k)=i \gamma_{5} X_{\mu_{1} \ldots \mu_{J}}^{(J)}(k), \\
& \widehat{Q}_{\mu_{1} \ldots \mu_{J}}^{(1, J+1, J)}(k)=\gamma_{\alpha}^{\perp} X_{\mu_{1} \ldots \mu_{J} \alpha}^{(J+1)}(k), \\
& \widehat{Q}_{\mu_{1} \ldots \mu_{J}}^{(1, J, J)}(k)=\varepsilon_{\alpha \nu_{1} \nu_{2} \nu_{3}} \gamma_{\alpha}^{\perp} P_{\nu_{1}} Z_{\nu_{2} \mu_{1} \ldots \mu_{J}, \nu_{3}}^{(J)}(k), \\
& \widehat{Q}_{\mu_{1} \ldots \mu_{J}}^{(1, J-1, J)}(k)=\gamma_{\alpha}^{\perp} Z_{\mu_{1} \ldots \mu_{J}, \alpha}^{(J-1)}(k) .
\end{aligned}
$$

For these operators, the wave functions read:

for $J=L+1$,

$$
\widehat{\Psi}_{(n) \mu_{1} \ldots \mu_{J}}^{(1, J-1, J)}(k)=\widehat{Q}_{\mu_{1} \ldots \mu_{J}}^{(1, J-1, J)}(k) \psi_{n}^{(1, J-1, J)}\left(k^{2}\right),
$$

for $J=L-1$,

$$
\widehat{\Psi}_{(n) \mu_{1} \ldots \mu_{J}}^{(1, J+1, J)}(k)=\widehat{Q}_{\mu_{1} \ldots \mu_{J}}^{(1, J+1, J)}(k) \psi_{n}^{(1, J+1, J)}\left(k^{2}\right),
$$

for $S=1, L=J \pm 1, J$,

$$
\widehat{\Psi}_{(n) \mu_{1} \ldots \mu_{J}}^{(1,(J \pm 1), J)}(k)=A_{j} \widehat{\Psi}_{(n) \mu_{1} \ldots \mu_{J}}^{(1, J-1, J)}(k)+B_{j} \widehat{\Psi}_{(n) \mu_{1} \ldots \mu_{J}}^{(1, J+1, J)}(k) .
$$

where $A_{j}$ and $B_{j}$ are the mixing coefficients with $j=1,2$.

These wave functions are normalized as follows:

$$
\begin{gathered}
\int \frac{d^{3} k}{(2 \pi)^{3} k_{0}}(-1) S p\left[\widehat{\Psi}_{\left(n^{\prime}\right) \mu_{1} \ldots \mu_{J}}^{\left(S^{\prime}, L_{j^{\prime}}, J^{\prime}\right)}(k)\left(\widehat{k}_{1}+m\right) \widehat{\Psi}_{(n) \mu_{1} \ldots \mu_{J}}^{\left(S, L_{j}, J\right)}(k)\left(-\widehat{k}_{2}+m\right)\right] \\
=(-1)^{J} \delta_{S^{\prime}, S} \delta_{L_{j^{\prime}}^{\prime}, L_{j}} \delta_{J^{\prime}, J} \delta_{n^{\prime}, n} .
\end{gathered}
$$




\section{A. Equation for $(S=0, J=L)$-state}

The equation for the state with $S=0, J=L$ reads:

$$
\begin{gathered}
\left(s-M^{2}\right) X_{\mu_{1} \ldots \mu_{J}}^{(J)}(k) S p\left[i \gamma_{5}\left(\widehat{k}_{1}+m\right) i \gamma_{5}\left(-\widehat{k}_{2}+m\right)\right] \times \\
\times X_{\mu_{1} \ldots \mu_{J}}^{(J)}(k) \psi_{n}^{(0, J, J)}\left(k^{2}\right)=X_{\mu_{1} \ldots \mu_{J}}^{(J)}(k) \sum_{c} S p\left[\widehat{F}_{c}\left(\widehat{k}_{1}+m\right) i \gamma_{5}\left(-\widehat{k}_{2}+m\right)\right] \times \\
\times \int \frac{d^{3} k^{\prime}}{(2 \pi)^{3} k_{0}^{\prime}} V_{c}\left(s, s^{\prime},\left(k k^{\prime}\right)\right) S p\left[i \gamma_{5}\left(\widehat{k}_{1}^{\prime}+m^{\prime}\right) \widehat{F}_{c}\left(-\widehat{k}_{2}^{\prime}+m^{\prime}\right)\right] \times \\
\times X_{\mu_{1} \ldots \mu_{J}}^{(J)}\left(k^{\prime}\right) \psi_{n}^{(0, J, J)}\left(k^{\prime 2}\right) .
\end{gathered}
$$

Now consider the left-hand side of the Eq. (89). Using the traces presented in Appendix B and convolution of operators from Appendix C, we have:

$$
\begin{gathered}
X_{\mu_{1} \ldots \mu_{J}}^{(J)}(k) S p\left[i \gamma_{5}\left(\widehat{k}_{1}+m\right) i \gamma_{5}\left(-\widehat{k}_{2}+m\right)\right] X_{\mu_{1} \ldots \mu_{J}}^{(J)}(k)= \\
=X_{\mu_{1} \ldots \mu_{J}}^{(J)}(k)(-2 s) X_{\mu_{1} \ldots \mu_{J}}^{(J)}(k)=-2 s \alpha(J) k^{2 J} .
\end{gathered}
$$

The right-hand side of the equation is calculated in two steps: first, we summarize with respect to $c$ :

$$
\begin{gathered}
A\left(s, s^{\prime},\left(k k^{\prime}\right)\right)=\sum_{c=T, A, P} A_{c}\left(s, s^{\prime},\left(k k^{\prime}\right)\right) V_{c}\left(s, s^{\prime},\left(k k^{\prime}\right)\right)= \\
=\sum_{c=T, A, P} S p\left[\widehat{F}_{c}\left(\widehat{k}_{1}+m\right) i \gamma_{5}\left(-\widehat{k}_{2}+m\right)\right] S p\left[i \gamma_{5}\left(\widehat{k}_{1}^{\prime}+m^{\prime}\right) \widehat{F}_{c}\left(-\widehat{k}_{2}^{\prime}+m^{\prime}\right)\right] \times \\
\times V_{c}\left(s, s^{\prime},\left(k k^{\prime}\right)\right) .
\end{gathered}
$$

In Appendix B the trace calculations are presented, and the values $A_{c}\left(s, s^{\prime},\left(k k^{\prime}\right)\right)$ are given. In this way, the sum is written as follows:

$$
\begin{aligned}
& A\left(s, s^{\prime},\left(k k^{\prime}\right)\right)=\sum_{c=T, A, P} A_{c}\left(s, s^{\prime},\left(k k^{\prime}\right)\right) V_{c}\left(s, s^{\prime},\left(k k^{\prime}\right)\right)=-4 \sqrt{s s^{\prime}} \times \\
& \times\left[\sqrt{s s^{\prime}} V_{P}\left(s, s^{\prime},\left(k k^{\prime}\right)\right)+4 m m^{\prime} V_{A}\left(s, s^{\prime},\left(k k^{\prime}\right)\right)+8\left(k k^{\prime}\right) V_{T}\left(s, s^{\prime},\left(k k^{\prime}\right)\right)\right] .
\end{aligned}
$$

At the second step, the convolution of operators is performed by using equations of Appendix $\mathrm{C}$ and recurrent formulae for the Legendre polynomials:

$$
z P_{J}(z)=\frac{J+1}{2 J+1} P_{J+1}(z)+\frac{J}{2 J+1} P_{J-1}(z)
$$

that allows us to write the Bethe-Salpeter equation in terms of the Legendre polynomials (recall that $z=$ $\left(k k^{\prime}\right) /\left(\sqrt{k^{2}} \sqrt{k^{\prime 2}}\right)$ and $\left.\sqrt{k^{2}}=i \sqrt{s / 4-m^{2}}, \sqrt{k^{\prime 2}}=i \sqrt{s^{\prime} / 4-m^{\prime 2}}\right)$. For the exceptional case $J=0$ we put $P_{-1}(z)=0$ As a result we get:

$$
X_{\mu_{1} \ldots \mu_{J}}^{(J)}(k) A\left(s, s^{\prime},\left(k k^{\prime}\right)\right) X_{\mu_{1} \ldots \mu_{J}}^{(J)}\left(k^{\prime}\right)=\alpha(J)\left(\sqrt{k^{2}} \sqrt{k^{\prime 2}}\right)^{J}\left(-4 \sqrt{s s^{\prime}}\right) \times
$$




$$
\begin{gathered}
\times\left[8 \frac{J+1}{2 J+1} \sqrt{k^{2}} \sqrt{k^{\prime 2}} P_{J+1}(z) V_{T}\left(s, s^{\prime},\left(k k^{\prime}\right)\right)+\right. \\
+\left(\sqrt{s s^{\prime}} V_{P}\left(s, s^{\prime},\left(k k^{\prime}\right)\right)+4 m m^{\prime} V_{A}\left(s, s^{\prime},\left(k k^{\prime}\right)\right)\right) P_{J}(z)+ \\
\left.+8 \frac{J}{2 J+1} \sqrt{k^{2}} \sqrt{k^{\prime 2}} P_{J-1}(z) V_{T}\left(s, s^{\prime},\left(k k^{\prime}\right)\right)\right] .
\end{gathered}
$$

Substituting the obtained expressions into Eq. (89), we obtain:

$$
\begin{gathered}
\left(s-M^{2}\right)(-2 s) \alpha(J) k^{2 J} \psi_{n}^{(0, J, J)}\left(k^{2}\right)=\int \frac{d^{3} k^{\prime}}{(2 \pi)^{3} k_{0}^{\prime}} \times \\
\times\left(-4 \sqrt{s s^{\prime}}\right) \alpha(J)\left(\sqrt{k^{2}} \sqrt{k^{\prime 2}}\right)^{J}\left[8 \frac{J+1}{2 J+1} \sqrt{k^{2}} \sqrt{k^{\prime 2}} P_{J+1}(z) V_{T}\left(s, s^{\prime},\left(k k^{\prime}\right)\right)+\right. \\
+\left(\sqrt{s s^{\prime}} V_{P}\left(s, s^{\prime},\left(k k^{\prime}\right)\right)+4 m m^{\prime} V_{A}\left(s, s^{\prime},\left(k k^{\prime}\right)\right)\right) P_{J}(z)+ \\
\left.+8 \frac{J}{2 J+1} \sqrt{k^{2}} \sqrt{k^{\prime 2}} P_{J-1}(z) V_{T}\left(s, s^{\prime},\left(k k^{\prime}\right)\right)\right] \psi_{n}^{(0, J, J)}\left(k^{\prime 2}\right) .
\end{gathered}
$$

Expanding the interaction block in the Legendre polynomial series,

$$
\begin{aligned}
& V_{c}\left(s, s^{\prime},\left(k k^{\prime}\right)\right)=\sum_{J} V_{c}^{(J)}\left(s, s^{\prime}\right) P_{J}(z)= \\
& =\sum_{J} \widetilde{V}_{c}^{(J)}\left(s, s^{\prime}\right) \alpha(J)\left(-\sqrt{k^{2}} \sqrt{k^{\prime 2}}\right)^{J} P_{J}(z),
\end{aligned}
$$

and integrating over angle variables in the right-hand side by taking into account the standard normalization condition $\int_{-1}^{1} d z / 2 P_{J}^{2}(z)=1 /(2 J+1)$, we have finally:

$$
\begin{gathered}
\left(s-M^{2}\right) \psi_{n}^{(0, J, J)}(s)=\int_{4 m^{\prime 2}}^{\infty} \frac{d s^{\prime}}{\pi} \rho\left(s^{\prime}\right)\left(-k^{\prime 2}\right)^{J} 2 \sqrt{\frac{s^{\prime}}{s}} \times \\
\times\left[-8 \frac{J+1}{2 J+1} \xi(J+1) k^{2} k^{\prime 2} \widetilde{V}_{T}^{(J+1)}\left(s, s^{\prime}\right)+\sqrt{s s^{\prime}} \xi(J) \widetilde{V}_{P}^{(J)}\left(s, s^{\prime}\right)+\right. \\
\left.+4 m m^{\prime} \xi(J) \widetilde{V}_{A}^{(J)}\left(s, s^{\prime}\right)-8 \frac{J}{2 J+1} \xi(J-1) \widetilde{V}_{T}^{(J-1)}\left(s, s^{\prime}\right)\right] \psi_{n}^{(0, J, J)}\left(s^{\prime}\right) .
\end{gathered}
$$

where:

$$
\begin{gathered}
\int_{-1}^{1} \frac{d z}{2} P_{J}(z) V_{c}\left(s, s^{\prime},\left(k k^{\prime}\right)\right)=\int_{-1}^{1} \frac{d z}{2} P_{J}(z) \sum_{J^{\prime}} \widetilde{V}_{c}^{\left(J^{\prime}\right)}\left(s, s^{\prime}\right) P_{J^{\prime}}(z) \alpha\left(J^{\prime}\right) \times \\
\times\left(-\sqrt{k^{2}} \sqrt{k^{\prime 2}}\right)^{J^{\prime}}=\frac{\alpha(J)}{2 J+1} \widetilde{V}_{c}^{(J)}\left(s, s^{\prime}\right)\left(-\sqrt{k^{2}} \sqrt{k^{\prime 2}}\right)^{J}= \\
=\xi(J) \widetilde{V}_{c}^{(J)}\left(s, s^{\prime}\right)\left(-\sqrt{k^{2}} \sqrt{k^{\prime 2}}\right)^{J}, \\
\xi(J)=\frac{\alpha(J)}{2 J+1}=\frac{(2 J-1) ! !}{(2 J+1) \cdot J !} .
\end{gathered}
$$




\section{Equation for the pion $\left(M^{2}, n\right)$-trajectory}

The pion states which belong to the pion $\left(M^{2}, n\right)$-trajectory obey the following equation:

$$
\begin{gathered}
\left(s-M^{2}\right) \psi_{\pi, n}^{(0,0,0)}(s)=\int_{4 m^{2}}^{\infty} \frac{d s^{\prime}}{\pi} \rho\left(s^{\prime}\right) 2 \sqrt{\frac{s^{\prime}}{s}} \times \\
\times\left[-\frac{8}{3} k^{2} k^{\prime 2} \widetilde{V}_{T}^{(1)}\left(s, s^{\prime}\right)+\sqrt{s s^{\prime}} \widetilde{V}_{P}^{(0)}\left(s, s^{\prime}\right)+4 m^{2} \widetilde{V}_{A}^{(0)}\left(s, s^{\prime}\right)\right] \psi_{\pi, n}^{(0,0,0)}\left(s^{\prime}\right) .
\end{gathered}
$$

Recall that $n$ is the radial quantum number, and the following states with different $n$ are located on the discussed $\left(M^{2}, n\right)$-trajectory: $\pi(140)$ with $n=1, \pi(1300)$ with $n=2, \pi(1800)$ with $n=3, \pi(2070)$ with $n=4, \pi(2360)$ with $n=5$, and so on.

The wave functions of the states laying on the $\left(M^{2}, n\right)$-trajectory satisfy the orthogonality/normalization constraint:

$$
\int_{4 m^{2}}^{\infty} \frac{d s}{\pi} \rho(s) 2 s \psi_{\pi, n^{\prime}}^{(0,0)}(s) \psi_{\pi, n}^{(0,0,0)}(s)=\delta_{n^{\prime}, n} .
$$

The factor $2 s$ is due to summing over the spin variables of quarks.

Expanding the interaction block over full set of the radial wave functions, we can transform (97) into a system of the linear equations of the type of (58).

\section{Equation for the $\eta\left(M^{2}, n\right)$-trajectory}

The $\eta$-states have two components, $n \bar{n}=(u \bar{u}+d \bar{d}) / \sqrt{2}$ and $s \bar{s}$. We write $\eta_{n}=\cos \Theta_{n} n \bar{n}+\sin \Theta_{n} s \bar{s}$, and $\eta_{n}^{\prime}=-\sin \Theta_{n} n \bar{n}+\cos \Theta_{n} s \bar{s}$. For the lightest mesons $\eta(550)$ and $\eta^{\prime}(958)$, one has $\cos \Theta_{1}=\simeq 0.8$ and $\sin \Theta_{1} \simeq-0.6$.

Correspondingly, we have two equations for the wave functions which describe the $n \bar{n}$ and $s \bar{s}$ components:

$$
\begin{gathered}
\left(s-M^{2}\right) \psi_{\eta(n \bar{n}), n}^{(0,0,0)}(s) \cos \Theta_{n}=\int_{4 m^{2}}^{\infty} \frac{d s^{\prime}}{\pi} \rho\left(s^{\prime}\right) 2 \sqrt{\frac{s^{\prime}}{s}} \times \\
\times\left[-\frac{8}{3} k^{2} k^{\prime 2} \widetilde{V}_{(n \bar{n} \rightarrow n \bar{n}), T}^{(1)}\left(s, s^{\prime}\right)+\sqrt{s s^{\prime}} \widetilde{V}_{(n \bar{n} \rightarrow n \bar{n}), P}^{(0)}\left(s, s^{\prime}\right)+4 m^{2} \widetilde{V}_{(n \bar{n} \rightarrow n \bar{n}), A}^{(0)}\left(s, s^{\prime}\right)\right] \times \\
\times \psi_{\eta(n \bar{n}), n}^{(0,0,0)}\left(s^{\prime}\right) \cos \Theta_{n}+\int_{4 m_{s}^{2}}^{\infty} \frac{d s^{\prime}}{\pi} \rho_{s}\left(s^{\prime}\right) 2 \sqrt{\frac{s^{\prime}}{s}}\left[-\frac{8}{3} k^{2} k_{s}^{\prime 2} \widetilde{V}_{(s \bar{s} \rightarrow n \bar{n}), T}^{(1)}\left(s, s^{\prime}\right)+\right. \\
\left.+\sqrt{s s^{\prime}} \widetilde{V}_{(s \bar{s} \rightarrow n \bar{n}), P}^{(0)}\left(s, s^{\prime}\right)+4 m m_{s} \widetilde{V}_{(s \bar{s} \rightarrow n \bar{n}), A}^{(0)}\left(s, s^{\prime}\right)\right] \psi_{\eta(s \bar{s}), n}^{(0,0,0)}\left(s^{\prime}\right) \sin \Theta_{n}
\end{gathered}
$$

where $\rho_{s}\left(s^{\prime}\right)$ refers to the $s \bar{s}$ phase space and $\sqrt{k_{s}^{\prime 2}}=i \sqrt{s^{\prime} / 4-m_{s}^{2}}$. The second equation, for the $s \bar{s}$-component, reads:

$$
\begin{gathered}
\left(s-M^{2}\right) \psi_{\eta(s \bar{s}), n}^{(0,0) 0)}(s) \sin \Theta_{n}=\int_{4 m^{2}}^{\infty} \frac{d s^{\prime}}{\pi} \rho\left(s^{\prime}\right) 2 \sqrt{\frac{s^{\prime}}{s}} \times \\
{\left[-\frac{8}{3} k_{s}^{2} k^{\prime 2} \widetilde{V}_{(n \bar{n} \rightarrow s \bar{s}), T}^{(1)}\left(s, s^{\prime}\right)+\sqrt{s s^{\prime}} \widetilde{V}_{(n \bar{n} \rightarrow s \bar{s}), P}^{(0)}\left(s, s^{\prime}\right)+4 m m_{s} \widetilde{V}_{(n \bar{n} \rightarrow s \bar{s}), A}^{(0)}\left(s, s^{\prime}\right)\right] \times}
\end{gathered}
$$




$$
\begin{aligned}
& \times \psi_{\eta(n \bar{n}), n}^{(0,0,0)}\left(s^{\prime}\right) \cos \Theta_{n}+\int_{4 m_{s}^{2}}^{\infty} \frac{d s^{\prime}}{\pi} \rho_{s}\left(s^{\prime}\right) 2 \sqrt{\frac{s^{\prime}}{s}}\left[-\frac{8}{3} k_{s}^{2} k_{s}^{\prime 2} \widetilde{V}_{(s \bar{s} \rightarrow s \bar{s}), T}^{(1)}\left(s, s^{\prime}\right)+\right. \\
& \left.\quad+\sqrt{s s^{\prime}} \widetilde{V}_{(s \bar{s} \rightarrow s \bar{s}), P}^{(0)}\left(s, s^{\prime}\right)+4 m_{s}^{2} \widetilde{V}_{(s \bar{s} \rightarrow s \bar{s}), A}^{(0)}\left(s, s^{\prime}\right)\right] \psi_{\eta(s \bar{s}), n}^{(0,0,0)}\left(s^{\prime}\right) \sin \Theta_{n} .
\end{aligned}
$$

The wave functions $\psi_{\eta(n \bar{n}), n}^{(0,0,0)}(s)$ and $\psi_{\eta(s \bar{s}), n}^{(0,0,0)}(s)$ satisfy the normalization condition within an obvious change of the integration region for the $s \bar{s}$ component: $4 m^{2} \rightarrow 4 m_{s}^{2}$.

The following states are located on the $\eta$ and $\eta^{\prime}\left(M^{2}, n\right)$-trajectories:

1) $\eta$-trajectory: $\eta(550)$ with $n=1, \eta(1295)$ with $n=2, \eta(1700)$ with $n=3, \eta(2010)$ with $n=4, \eta(2320)$ with $n=5$, and so on.

2) $\eta^{\prime}$-trajectory: $\eta^{\prime}(958)$ with $n=1, \eta(1440)$ with $n=2, \eta(1820)$ with $n=3$, and so on.

\section{Equation for the $b_{1}\left(M^{2}, n\right)$-trajectory}

The equation for the states with $S=0, L=1, J=1$ reads:

$$
\begin{gathered}
\left(s-M^{2}\right) \psi_{b_{1}, n}^{(0,1,1)}(s)=-\int_{4 m^{2}}^{\infty} \frac{d s^{\prime}}{\pi} \rho\left(s^{\prime}\right) k^{\prime 2} \frac{2}{3} \sqrt{\frac{s^{\prime}}{s}} \times \\
\times\left[-\frac{24}{5} k^{2} k^{\prime 2} \widetilde{V}_{T}^{(2)}\left(s, s^{\prime}\right)+\sqrt{s s^{\prime}} \widetilde{V}_{P}^{(1)}\left(s, s^{\prime}\right)+4 m^{2} \widetilde{V}_{A}^{(1)}\left(s, s^{\prime}\right)-8 \widetilde{V}_{T}^{(0)}\left(s, s^{\prime}\right)\right] \times \\
\times \psi_{b_{1}, n}^{(0,1,1)}\left(s^{\prime}\right) .
\end{gathered}
$$

The following states are located on the $b_{1}\left(M^{2}, n\right)$-trajectories: $b_{1}(1235)$ with $n=1, b_{1}(1640)$ at $n=2, b_{1}(1970)$ with $n=3, b_{1}(2210)$ with $n=4$, and so on.

The wave functions of the $b_{1}$-states laying on the $\left(M^{2}, n\right)$-trajectory satisfy the orthogonality/normalization constraint:

$$
\int_{4 m^{2}}^{\infty} \frac{d s}{\pi} \rho(s) 2 s k^{2} \psi_{b_{1}, n^{\prime}}^{(0,1,1)}(s) \psi_{b_{1}, n}^{(0,1,1)}(s)=\delta_{n^{\prime}, n} .
$$

\section{Equation for the $h_{1}\left(M^{2}, n\right)$-trajectory}

The $h_{1}$-states have two components, $n \bar{n}=(u \bar{u}+d \bar{d}) / \sqrt{2}$ and $s \bar{s}$; we write $h_{1, n}=\cos \Theta_{n} n \bar{n}+\sin \Theta_{n} s \bar{s}$. Correspondingly, we have two equations for the wave-function $n \bar{n}$-component:

$$
\begin{gathered}
\left(s-M^{2}\right) \psi_{h_{1}(n \bar{n}), n}^{(0,1,1)}(s) \cos \Theta_{n}=-\int_{4 m^{2}}^{\infty} \frac{d s^{\prime}}{\pi} \rho\left(s^{\prime}\right) k^{\prime 2} \frac{2}{3} \sqrt{\frac{s^{\prime}}{s}} \times \\
\times\left[-\frac{24}{5} k^{2} k^{\prime 2} \widetilde{V}_{(n \bar{n} \rightarrow n \bar{n}), T}^{(2)}\left(s, s^{\prime}\right)+\sqrt{s s^{\prime}} \widetilde{V}_{(n \bar{n} \rightarrow n \bar{n}), P}^{(1)}\left(s, s^{\prime}\right)+\right. \\
\left.+4 m^{2} \widetilde{V}_{(n \bar{n} \rightarrow n \bar{n}), A}^{(1)}\left(s, s^{\prime}\right)-8 \widetilde{V}_{(n \bar{n} \rightarrow n \bar{n}), T}^{(0)}\left(s, s^{\prime}\right)\right] \psi_{h_{1}(n \bar{n}), n}^{(0,1,1)}\left(s^{\prime}\right) \cos \Theta_{n}-
\end{gathered}
$$




$$
\begin{gathered}
-\int_{4 m_{s}^{2}}^{\infty} \frac{d s^{\prime}}{\pi} \rho_{s}\left(s^{\prime}\right) k_{s}^{\prime 2} \frac{2}{3} \sqrt{\frac{s^{\prime}}{s}}\left[-\frac{24}{5} k^{2} k_{s}^{\prime 2} \widetilde{V}_{(s \bar{s} \rightarrow n \bar{n}), T}^{(2)}\left(s, s^{\prime}\right)+\sqrt{s s^{\prime}} \widetilde{V}_{(s \bar{s} \rightarrow n \bar{n}), P}^{(1)}\left(s, s^{\prime}\right)+\right. \\
\left.+4 m m_{s} \widetilde{V}_{(s \bar{s} \rightarrow n \bar{n}), A}^{(1)}\left(s, s^{\prime}\right)-8 \widetilde{V}_{(s \bar{s} \rightarrow n \bar{n}), T}^{(0)}\left(s, s^{\prime}\right)\right] \psi_{h_{1}(s \bar{s}), n}^{(0,1,1)}\left(s^{\prime}\right) \sin \Theta_{n},
\end{gathered}
$$

where $\rho_{s}\left(s^{\prime}\right)$ refers to the $s \bar{s}$ phase space and $\sqrt{k_{s}^{\prime 2}}=i \sqrt{s^{\prime} / 4-m_{s}^{2}}$. For the $s \bar{s}$-component we have:

$$
\begin{gathered}
\left(s-M^{2}\right) \psi_{h_{1}(s \bar{s}), n}^{(0,1,1)}(s) \sin \Theta_{n}=-\int_{4 m^{2}}^{\infty} \frac{d s^{\prime}}{\pi} \rho\left(s^{\prime}\right) k^{2} \frac{2}{3} \sqrt{\frac{s^{\prime}}{s}} \times \\
\times\left[-\frac{24}{5} k_{s}^{2} k^{\prime 2} \widetilde{V}_{(n \bar{n} \rightarrow s \bar{s}), T}^{(2)}\left(s, s^{\prime}\right)+\sqrt{s s^{\prime}} \widetilde{V}_{(n \bar{n} \rightarrow s \bar{s}), P}^{(1)}\left(s, s^{\prime}\right)+\right. \\
\left.+4 m m_{s} \widetilde{V}_{(n \bar{n} \rightarrow s \bar{s}), A}^{(1)}\left(s, s^{\prime}\right)-8 \widetilde{V}_{(n \bar{n} \rightarrow s \bar{s}), T}^{(0)}\left(s, s^{\prime}\right)\right] \psi_{h_{1}(n \bar{n}), n}^{(0,1,1)}\left(s^{\prime}\right) \cos \Theta_{n}- \\
\int_{4 m_{s}^{2}}^{\infty} \frac{d s^{\prime}}{\pi} \rho_{s}\left(s^{\prime}\right) k_{s}^{\prime 2} \frac{2}{3} \sqrt{\frac{s^{\prime}}{s}}\left[-\frac{24}{5} k_{s}^{2} k_{s}^{\prime 2} \widetilde{V}_{(s \bar{s} \rightarrow s \bar{s}), T}^{(2)}\left(s, s^{\prime}\right)+\sqrt{s s^{\prime}} \widetilde{V}_{(s \bar{s} \rightarrow s \bar{s}), P}^{(1)}\left(s, s^{\prime}\right)+\right. \\
\left.+4 m_{s}^{2} \widetilde{V}_{(s \bar{s} \rightarrow s \bar{s}), A}^{(1)}\left(s, s^{\prime}\right)-8 \widetilde{V}_{(s \bar{s} \rightarrow s \bar{s}), T}^{(0)}\left(s, s^{\prime}\right)\right] \psi_{h_{1}(s \bar{s}), n}^{(0,1,1)}\left(s^{\prime}\right) \sin \Theta_{n} .
\end{gathered}
$$

The wave functions $\psi_{h_{1}(n \bar{n}), n}^{(0,1,1)}(s)$ and $\psi_{h_{1}(s \bar{s}), n}^{(0,1,1)}(s)$ satisfy the normalization condition within the obvious change the integration region for the $s \bar{s}$ component: $4 m^{2} \rightarrow 4 m_{s}^{2}$.

The following states are located on the $h_{1}\left(M^{2}, n\right)$-trajectories:

1) $h_{1}(1170)$ with $n=1, h_{1}(1600)$ with $n=2, h_{1}(2000)$ with $n=3, h_{1}(2270)$ with $n=4$, and so on;

2) $h_{1}(1390)$ with $n=1, h_{1}(1780)$ with $n=2, h_{1}(2120)$ with $n=3$, and so on.

\section{Equation for the $\pi_{2}\left(M^{2}, n\right)$-trajectories}

The equation for the $\pi_{2}$-states $(S=0, L=2, J=2)$ reads:

$$
\begin{gathered}
\left(s-M^{2}\right) \psi_{\pi_{2}, n}^{(0,2,2)}(s)=\int_{4 m^{2}}^{\infty} \frac{d s^{\prime}}{\pi} \rho\left(s^{\prime}\right) k^{\prime 4} \frac{3}{5} \sqrt{\frac{s^{\prime}}{s}} \times \\
\times\left[-\frac{40}{7} k^{2} k^{\prime 2} \widetilde{V}_{T}^{(3)}\left(s, s^{\prime}\right)+\sqrt{s s^{\prime}} \widetilde{V}_{P}^{(2)}\left(s, s^{\prime}\right)+4 m^{2} \widetilde{V}_{A}^{(2)}\left(s, s^{\prime}\right)-\frac{32}{9} \widetilde{V}_{T}^{(1)}\left(s, s^{\prime}\right)\right] \times \\
\times \psi_{\pi_{2}, n}^{(0,2,2)}\left(s^{\prime}\right) .
\end{gathered}
$$

The following states are located on the $\pi_{2}\left(M^{2}, n\right)$-trajectory [15]: $\pi_{2}(1670)$ with $n=1, \pi_{2}(2005)$ with $n=2, \pi_{2}(2245)$ with $n=3$, and so on.

The wave functions of the $\pi_{2}$-states satisfy the orthogonality/normalization constraint:

$$
\int_{4 m^{2}}^{\infty} \frac{d s}{\pi} \rho(s) k^{4} 2 s \alpha(2) \psi_{\pi, n^{\prime}}^{(0,2,2)}(s) \psi_{\pi, n}^{(0,2,2)}(s)=\delta_{n^{\prime}, n} .
$$

where $\alpha(2)$ is determined by Eq. (63). 
The $\eta_{2}$-states have two components, $n \bar{n}=(u \bar{u}+d \bar{d}) / \sqrt{2}$ and $s \bar{s}$. We write $\eta_{2, n}=\cos \Theta_{n} n \bar{n}+\sin \Theta_{n} s \bar{s}$, and, correspondingly, we have two equations for the wave functions:

$$
\begin{gathered}
\left(s-M^{2}\right) \psi_{\eta_{2}(n \bar{n}), n}^{(0,2,2)}(s) \cos \Theta_{n}=\int_{4 m^{2}}^{\infty} \frac{d s^{\prime}}{\pi} \rho\left(s^{\prime}\right) k^{\prime 4} \frac{3}{5} \sqrt{\frac{s^{\prime}}{s}} \times \\
\times\left[-\frac{40}{7} k^{2} k^{\prime 2} \widetilde{V}_{(n \bar{n} \rightarrow n \bar{n}), T}^{(3)}\left(s, s^{\prime}\right)+\sqrt{s s^{\prime}} \widetilde{V}_{(n \bar{n} \rightarrow n \bar{n}), P}^{(2)}\left(s, s^{\prime}\right)+\right. \\
\left.+4 m^{2} \widetilde{V}_{(n \bar{n} \rightarrow n \bar{n}), A}^{(2)}\left(s, s^{\prime}\right)-\frac{32}{9} \widetilde{V}_{(n \bar{n} \rightarrow n \bar{n}), T}^{(1)}\left(s, s^{\prime}\right)\right] \psi_{\eta_{2}(n \bar{n}), n}^{(0,2,2)}\left(s^{\prime}\right) \cos \Theta_{n}+ \\
+\int_{4 m_{s}^{2}}^{\infty} \frac{d s^{\prime}}{\pi} \rho_{s}\left(s^{\prime}\right) k_{s}^{\prime 4} \frac{3}{5} \sqrt{\frac{s^{\prime}}{s}}\left[-\frac{40}{7} k^{2} k_{s}^{\prime 2} \widetilde{V}_{(s \bar{s} \rightarrow n \bar{n}), T}^{(3)}\left(s, s^{\prime}\right)+\sqrt{s s^{\prime}} \widetilde{V}_{(s \bar{s} \rightarrow n \bar{n}), P}^{(2)}\left(s, s^{\prime}\right)+\right. \\
\left.+4 m m_{s} \widetilde{V}_{(s \bar{s} \rightarrow n \bar{n}), A}^{(2)}\left(s, s^{\prime}\right)-\frac{32}{9} \widetilde{V}_{(s \bar{s} \rightarrow n \bar{n}), T}^{(1)}\left(s, s^{\prime}\right)\right] \psi_{\eta_{2}(s \bar{s}), n}^{(0,2,2)}\left(s^{\prime}\right) \sin \Theta_{n},
\end{gathered}
$$

and

$$
\begin{gathered}
\left(s-M^{2}\right) \psi_{\eta_{2}(s \bar{s}), n}^{(0,2,2)}(s) \sin \Theta_{n}=\int_{4 m^{2}}^{\infty} \frac{d s^{\prime}}{\pi} \rho\left(s^{\prime}\right) k^{\prime 4} \frac{3}{5} \sqrt{\frac{s^{\prime}}{s}} \times \\
\times\left[-\frac{40}{7} k_{s}^{2} k^{\prime 2} \widetilde{V}_{(n \bar{n} \rightarrow s \bar{s}), T}^{(3)}\left(s, s^{\prime}\right)+\sqrt{s s^{\prime}} \widetilde{V}_{(n \bar{n} \rightarrow s \bar{s}), P}^{(2)}\left(s, s^{\prime}\right)+\right. \\
\left.+4 m m_{s} \widetilde{V}_{(n \bar{n} \rightarrow s \bar{s}), A}^{(2)}\left(s, s^{\prime}\right)-\frac{32}{9} \widetilde{V}_{(n \bar{n} \rightarrow s \bar{s}), T}^{(1)}\left(s, s^{\prime}\right)\right] \psi_{\eta_{2}(n \bar{n}), n}^{(0,2,2)}\left(s^{\prime}\right) \cos \Theta_{n}+ \\
+\int_{4 m_{s}^{2}}^{\infty} \frac{d s^{\prime}}{\pi} \rho_{s}\left(s^{\prime}\right) k_{s}^{\prime 4} \frac{3}{5} \sqrt{\frac{s^{\prime}}{s}}\left[-\frac{40}{7} k_{s}^{2} k_{s}^{\prime 2} \widetilde{V}_{(s \bar{s} \rightarrow s \bar{s}), T}^{(3)}\left(s, s^{\prime}\right)+\sqrt{s s^{\prime}} \widetilde{V}_{(s \bar{s} \rightarrow s \bar{s}), P}^{(2)}\left(s, s^{\prime}\right)+\right. \\
\left.+4 m_{s}^{2} \widetilde{V}_{(s \bar{s} \rightarrow s \bar{s}), A}^{(2)}\left(s, s^{\prime}\right)-\frac{32}{9} \widetilde{V}_{(s \bar{s} \rightarrow s \bar{s}), T}^{(1)}\left(s, s^{\prime}\right)\right] \psi_{\eta_{2}(s \bar{s}), n}^{(0,2,2)}\left(s^{\prime}\right) \sin \Theta_{n} .
\end{gathered}
$$

The wave functions $\psi_{\eta_{2}(n \bar{n}), n}^{(0,2,2)}(s)$ and $\psi_{\eta_{2}(s \bar{s}), n}^{(0,2)}(s)$ satisfy the normalization condition (106), with obvious change of the integration region for the $s \bar{s}$ component: $4 m^{2} \rightarrow 4 m_{s}^{2}$.

The following states are located on the $\eta_{2}\left(M^{2}, n\right)$-trajectory [15]: $\eta_{2}(1645)$ with $n=1, \eta_{2}(2030)$ with $n=2$, $\eta_{2}(2250)$ with $n=3$, and so on. 
The equation for the $b_{3}$-mesons $(S=0, L=3, J=3)$ is as follows:

$$
\begin{gathered}
\left(s-M^{2}\right) \psi_{b_{3}, n}^{(0,3,3)}(s)=-\int_{4 m^{2}}^{\infty} \frac{d s^{\prime}}{\pi} \rho\left(s^{\prime}\right) k^{\prime 6} \frac{5}{7} \sqrt{\frac{s^{\prime}}{s}} \times \\
\times\left[-\frac{56}{9} k^{2} k^{\prime 2} \widetilde{V}_{T}^{(2)}\left(s, s^{\prime}\right)+\sqrt{s s^{\prime}} \widetilde{V}_{P}^{(1)}\left(s, s^{\prime}\right)+4 m^{2} \widetilde{V}_{A}^{(1)}\left(s, s^{\prime}\right)-\frac{72}{25} \widetilde{V}_{T}^{(0)}\left(s, s^{\prime}\right)\right] \times \\
\times \psi_{b_{3}, n}^{(0,3,3)}\left(s^{\prime}\right) .
\end{gathered}
$$

According to [15], the following states are located on the $b_{3}\left(M^{2}, n\right)$-trajectory in the mass region below $2400 \mathrm{MeV}$ : $b_{3}(2020)$ with $n=1, b_{3}(2245)$ with $n=2$, and so on.

The wave functions of the states laying on the $\left(M^{2}, n\right)$-trajectory satisfy the orthogonality/normalization constraint:

$$
\int_{4 m^{2}}^{\infty} \frac{d s}{\pi} \rho(s) k^{6} 2 s \alpha(3) \psi_{b_{3}, n^{\prime}}^{(0,3,3)}(s) \psi_{b_{3}, n}^{(0,3,3)}(s)=\delta_{n^{\prime}, n} .
$$

The factor $\alpha(3)$ is given by the Eq. (63).

\section{B. Equation for the $(S=1, J=L)$-state}

The equation for the $(S=1, J=L)$ state reads:

$$
\begin{gathered}
\left(s-M^{2}\right) \varepsilon_{\beta \nu_{1} \nu_{2} \nu_{3}} P_{\nu_{1}} Z_{\nu_{2} \mu_{1} \cdots \mu_{J}, \nu_{3}}^{(J)}(k) S p\left[\gamma_{\alpha}^{\perp}\left(\widehat{k}_{1}+m\right) \gamma_{\beta}^{\perp}\left(-\widehat{k}_{2}+m\right)\right] \times \\
\times \varepsilon_{\alpha \xi_{1} \xi_{2} \xi_{3}} P_{\xi_{1}} Z_{\xi_{2} \mu_{1} \cdots \mu_{J}, \xi_{3}}^{(J)}(k) \psi_{n}^{(1, J, J)}(s)= \\
=\varepsilon_{\beta^{\prime} \nu_{1} \nu_{2} \nu_{3}} P_{\nu_{1}} Z_{\nu_{2} \mu_{1} \cdots \mu_{J}, \nu_{3}}^{(J)}(k) \sum_{c} S p\left[\widehat{F}_{c}\left(\widehat{k}_{1}+m\right) \gamma_{\beta^{\prime}}^{\perp}\left(-\widehat{k}_{2}+m\right)\right] \times \\
\times \int \frac{d^{3} k^{\prime}}{(2 \pi)^{3} k_{0}^{\prime}} V_{c}\left(s, s^{\prime},\left(k k^{\prime}\right)\right) S p\left[\gamma_{\alpha^{\prime}}^{\perp}\left(\widehat{k}_{1}^{\prime}+m^{\prime}\right) \widehat{F}_{c}\left(-\widehat{k}_{2}^{\prime}+m^{\prime}\right)\right] \times \\
\times \varepsilon_{\alpha^{\prime} \xi_{1} \xi_{2} \xi_{3}} P_{\xi_{1}} Z_{\xi_{2} \mu_{1} \cdots \mu_{J}, \xi_{3}}^{(J)}\left(k^{\prime}\right) \psi_{n}^{(1, J, J)}\left(s^{\prime}\right) .
\end{gathered}
$$

The left-hand side of the equation is calculated by using the trace and operator convolutions given in Appendices B and $\mathrm{C}$ :

$$
\begin{gathered}
\varepsilon_{\beta \nu_{1} \nu_{2} \nu_{3}} P_{\nu_{1}} Z_{\nu_{2} \mu_{1} \cdots \mu_{J}, \nu_{3}}^{(J)}(k) S p\left[\gamma_{\alpha}^{\perp}\left(\widehat{k}_{1}+m\right) \gamma_{\beta}^{\perp}\left(-\widehat{k}_{2}+m\right)\right] \times \\
\times \varepsilon_{\alpha \xi_{1} \xi_{2} \xi_{3}} P_{\xi_{1}} Z_{\xi_{2} \mu_{1} \cdots \mu_{J}, \xi_{3}}^{(J)}(k)=-2 s^{2} \frac{J(2 J+3)^{2}}{(J+1)^{3}} \alpha(J) k^{2 J} .
\end{gathered}
$$

As before, the right-hand side is calculated in two steps.

1) We calculate traces:

$$
B_{\beta^{\prime} \alpha^{\prime}}\left(s, s^{\prime},\left(k k^{\prime}\right)\right)=\sum_{c=T, A, V, S}\left(B_{c}\right)_{\beta^{\prime} \alpha^{\prime}}\left(s, s^{\prime},\left(k k^{\prime}\right)\right) V_{c}\left(s, s^{\prime},\left(k k^{\prime}\right)\right)=
$$




$$
\begin{gathered}
=\sum_{c=T, A, V, S} S p\left[\widehat{F}_{c}\left(\widehat{k}_{1}+m\right) \gamma_{\beta^{\prime}}^{\perp}\left(-\widehat{k}_{2}+m\right)\right] S p\left[\gamma_{\alpha^{\prime}}^{\perp}\left(\widehat{k}_{1}^{\prime}+m^{\prime}\right) \widehat{F}_{c}\left(-\widehat{k}_{2}^{\prime}+m^{\prime}\right)\right] \times \\
\times V_{c}\left(s, s^{\prime},\left(k k^{\prime}\right)\right) .
\end{gathered}
$$

Following the items presented in Appendix B, we write:

$$
\begin{aligned}
& B_{\beta^{\prime} \alpha^{\prime}}\left(s, s^{\prime},\left(k k^{\prime}\right)\right)=g_{\beta^{\prime} \alpha^{\prime}}^{\perp} 4 \sqrt{s s^{\prime}}\left[\sqrt{s s^{\prime}} V_{V}\left(s, s^{\prime},\left(k k^{\prime}\right)\right)+\right. \\
& \left.+8 m m^{\prime} V_{T}\left(s, s^{\prime},\left(k k^{\prime}\right)\right)+4 \sqrt{k^{2}} \sqrt{k^{\prime 2}} z V_{A}\left(s, s^{\prime},\left(k k^{\prime}\right)\right)\right]+ \\
& +64 m m^{\prime} k_{\beta^{\prime}}^{\perp} k_{\alpha^{\prime}}^{\prime \perp} V_{S}\left(s, s^{\prime},\left(k k^{\prime}\right)\right)-16 k_{\beta^{\prime}}^{\prime \perp} k_{\alpha^{\prime}}^{\perp} \sqrt{s s^{\prime}} V_{A}\left(s, s^{\prime},\left(k k^{\prime}\right)\right)+ \\
& +16\left[s^{\prime} k_{\beta^{\prime}}^{\perp} k_{\alpha^{\prime}}^{\perp}+s k_{\beta^{\prime}}^{\prime \perp} k_{\alpha^{\prime}}^{\prime \perp}+4 z \sqrt{k^{2}} \sqrt{k^{2}} k_{\beta^{\prime}}^{\perp} k_{\alpha^{\prime}}^{\prime \perp}\right] V_{V}\left(s, s^{\prime},\left(k k^{\prime}\right)\right) .
\end{aligned}
$$

2) The convolutions of the trace factor $B_{\beta^{\prime} \alpha^{\prime}}\left(s, s^{\prime},\left(k k^{\prime}\right)\right)$ with angular momentum wave functions are presented in Appendix C; we have:

$$
\begin{gathered}
\varepsilon_{\beta^{\prime} \nu_{1} \nu_{2} \nu_{3}} P_{\nu_{1}} Z_{\nu_{2} \mu_{1} \cdots \mu_{J}, \nu_{3}}^{(J)}(k) B_{\beta^{\prime} \alpha^{\prime}}\left(s, s^{\prime},\left(k k^{\prime}\right)\right) \times \\
\times \varepsilon_{\alpha^{\prime} \xi_{1} \xi_{2} \xi_{3}} P_{\xi_{1}} Z_{\xi_{2} \mu_{1} \cdots \mu_{J}, \xi_{3}}^{(J)}\left(k^{\prime}\right)=\alpha(J)\left(\sqrt{k^{2}} \sqrt{k^{\prime 2}}\right)^{J}\left(-4 s s^{\prime}\right) \frac{J(2 J+3)^{2}}{(J+1)^{3}} \times \\
\times\left[4 \frac{J}{2 J+1} \sqrt{k^{2}} \sqrt{k^{\prime 2}} P_{J+1}(z) V_{A}\left(s, s^{\prime},\left(k k^{\prime}\right)\right)+\left(\sqrt{s s^{\prime}} V_{V}\left(s, s^{\prime},\left(k k^{\prime}\right)\right)+\right.\right. \\
\left.\left.+8 m m^{\prime} V_{T}\left(s, s^{\prime},\left(k k^{\prime}\right)\right)\right) P_{J}(z)+4 \frac{J+1}{2 J+1} \sqrt{k^{2}} \sqrt{k^{\prime 2}} P_{J-1}(z) V_{A}\left(s, s^{\prime},\left(k k^{\prime}\right)\right)\right] .
\end{gathered}
$$

Inserting these expressions into Eq. (111), we obtain:

$$
\begin{gathered}
\left(s-M^{2}\right)\left(-2 s^{2}\right) k^{2 J} \psi_{n}^{(1, J, J)}(s)=\int \frac{d^{3} k^{\prime}}{(2 \pi)^{3} k_{0}^{\prime}}\left(-4 s s^{\prime}\right)\left(\sqrt{k^{2}} \sqrt{k^{\prime 2}}\right)^{J} \times \\
\times\left[4 \frac{J}{2 J+1} \sqrt{k^{2}} \sqrt{k^{\prime 2}} P_{J+1}(z) V_{A}\left(s, s^{\prime},\left(k k^{\prime}\right)\right)+\sqrt{s s^{\prime}} V_{V}\left(s, s^{\prime},\left(k k^{\prime}\right)\right) P_{J}(z)+\right. \\
\left.+8 m m^{\prime} V_{T}\left(s, s^{\prime},\left(k k^{\prime}\right)\right) P_{J}(z)+4 \frac{J+1}{2 J+1} \sqrt{k^{2}} \sqrt{k^{\prime 2}} P_{J-1}(z) V_{A}\left(s, s^{\prime},\left(k k^{\prime}\right)\right)\right] \times \\
\times \psi_{n}^{(1, J, J)}\left(s^{\prime}\right) .
\end{gathered}
$$

Expanding the interaction block according to (94) and integrating both sides over $\int_{-1}^{1} d z / 2$, we get:

$$
\begin{gathered}
\left(s-M^{2}\right) \psi_{n}^{(1, J, J)}(s)=\int_{4 m^{\prime 2}}^{\infty} \frac{d s^{\prime}}{\pi} \rho\left(s^{\prime}\right)\left(-k^{\prime 2}\right)^{J} 2 \frac{s^{\prime}}{s} \times \\
\times\left[-4 \frac{J}{2 J+1} \xi(J+1) k^{2} k^{\prime 2} \widetilde{V}_{A}^{(J+1)}\left(s, s^{\prime}\right)+\sqrt{s s^{\prime}} \xi(J) \widetilde{V}_{V}^{(J)}\left(s, s^{\prime}\right)+\right. \\
\left.+8 m m^{\prime} \xi(J) \widetilde{V}_{T}^{(J)}\left(s, s^{\prime}\right)-4 \frac{J+1}{2 J+1} \xi(J-1) \widetilde{V}_{A}^{(J-1)}\left(s, s^{\prime}\right)\right] \psi_{n}^{(1, J, J)}\left(s^{\prime}\right) .
\end{gathered}
$$

Normalization condition for the $(S=1, J=L)$ wave functions reads:

$$
\int_{4 m^{2}}^{\infty} \frac{d s}{\pi} \rho(s) k^{2 J} 2 s^{2} \frac{J(2 J+3)^{2}}{(J+1)^{3}} \alpha(J) \psi_{n^{\prime}}^{(1, J, J)}(s) \psi_{n}^{(1, J, J)}(s)=\delta_{n^{\prime}, n} .
$$


The $a_{1}$ states $(S=1, L=1, J=1)$ obey the Bethe-Salpeter equation:

$$
\begin{gathered}
\left(s-M^{2}\right) \psi_{a_{1}, n}^{(1,1,1)}(s)=-\int_{4 m^{2}}^{\infty} \frac{d s^{\prime}}{\pi} \rho\left(s^{\prime}\right) k^{\prime 2} \frac{2}{3} \frac{s^{\prime}}{s} \times \\
\times\left[-\frac{6}{5} k^{2} k^{\prime 2} \widetilde{V}_{A}^{(2)}\left(s, s^{\prime}\right)+\sqrt{s s^{\prime}} \widetilde{V}_{V}^{(1)}\left(s, s^{\prime}\right)+8 m^{2} \widetilde{V}_{T}^{(1)}\left(s, s^{\prime}\right)-8 \widetilde{V}_{A}^{(0)}\left(s, s^{\prime}\right)\right] \times \\
\times \psi_{a_{1}, n}^{(1,1,1)}\left(s^{\prime}\right) .
\end{gathered}
$$

The following states are located on the $a_{1}\left(M^{2}, n\right)$-trajectory [15]: $a_{1}(1230)$ with $n=1, a_{1}(1640)$ with $n=2$, $a_{1}(1960)$ with $n=3, a_{1}(2270)$ with $n=4$, and so on.

The wave functions of the $a_{1}$-states satisfy the orthogonality/normalization condition:

$$
\int_{4 m^{2}}^{\infty} \frac{d s}{\pi} \rho(s) k^{2} 2 s^{2} \frac{25}{8} \alpha(1) \psi_{a_{1}, n^{\prime}}^{(1,1,1)}(s) \psi_{a_{1}, n}^{(1,1,1)}(s)=\delta_{n^{\prime}, n} .
$$

\section{Equation for the $a_{3}\left(M^{2}, n\right)$-trajectory}

For the $a_{3}$-mesons $(S=1, L=3, J=3)$ the Bethe-Salpeter equation reads:

$$
\begin{gathered}
\left(s-M^{2}\right) \psi_{a_{3}, n}^{(1,3,3)}(s)=-\int_{4 m^{2}}^{\infty} \frac{d s^{\prime}}{\pi} \rho\left(s^{\prime}\right) k^{\prime 6} \frac{5}{7} \frac{s^{\prime}}{s} \times \\
\times\left[-\frac{7}{3} k^{2} k^{\prime 2} \widetilde{V}_{A}^{(4)}\left(s, s^{\prime}\right)+\sqrt{s s^{\prime}} \widetilde{V}_{V}^{(3)}\left(s, s^{\prime}\right)+8 m^{2} \widetilde{V}_{T}^{(3)}\left(s, s^{\prime}\right)-\frac{48}{25} \widetilde{V}_{A}^{(2)}\left(s, s^{\prime}\right)\right] \times \\
\times \psi_{a_{3}, n}^{(1,3,3)}\left(s^{\prime}\right) .
\end{gathered}
$$

Two $a_{3}$-states were seen: $a_{3}(2030)$ with $n=1, a_{3}(2275)$ with $n=2[15]$.

The orthogonality/normalization constraint reads:

$$
\int_{4 m^{2}}^{\infty} \frac{d s}{\pi} \rho(s) k^{6} s \frac{243}{32} \alpha(3) \psi_{a_{3}, n^{\prime}}^{(1,3,3)}(s) \psi_{a_{3}, n}^{(1,3,3)}(s)=\delta_{n^{\prime}, n} .
$$

\section{Equations for the $(S=1, J=L \pm 1)$-states}

We have two equations for two states with $S=1$ and $J=L \pm 1$ for $J>0$. Corresponding wave functions are denoted as $A_{j} \widehat{\Psi}_{(n) \mu_{1} \ldots \mu_{J}}^{(1, J-1, J)}(k)+B_{j} \widehat{\Psi}_{(n) \mu_{1} \ldots \mu_{J}}^{(1, J)}(k)$ with $j=1,2$. These wave functions are orthogonal to one another. Normalization and orthogonality conditions give three constraints for four mixing parameters $A_{j}$ and $B_{j}$.

Each wave function obeys two equations:

$$
\left(s-M^{2}\right) X_{\mu_{1} \ldots \mu_{J} \beta}^{(J+1)}(k) S p\left[\gamma_{\alpha}^{\perp}\left(\widehat{k}_{1}+m\right) \gamma_{\beta}^{\perp}\left(-\widehat{k}_{2}+m\right)\right] \times
$$




$$
\begin{gathered}
\times\left(A_{j} Z_{\mu_{1} \ldots \mu_{J}, \alpha}^{(J-1)}(k) \psi_{n}^{(1, J-1, J)}\left(k^{2}\right)+B_{j} X_{\mu_{1} \ldots \mu_{J} \alpha}^{(J+1)}(k) \psi_{n}^{(1, J+1, J)}\left(k^{2}\right)\right)= \\
=X_{\mu_{1} \ldots \mu_{J} \beta^{\prime}}^{(J+1)}(k) \sum_{c} S p\left[\widehat{F}_{c}\left(\widehat{k}_{1}+m\right) \gamma_{\beta^{\prime}}^{\perp}\left(-\widehat{k}_{2}+m\right)\right] \times \\
\times \int \frac{d^{3} k^{\prime}}{(2 \pi)^{3} k_{0}^{\prime}} V_{c}\left(s, s^{\prime},\left(k k^{\prime}\right)\right) S p\left[\gamma_{\alpha^{\prime}}^{\perp}\left(\widehat{k}_{1}^{\prime}+m^{\prime}\right) \widehat{F}_{c}\left(-\widehat{k}_{2}^{\prime}+m^{\prime}\right)\right] \times \\
\times\left(A_{j} Z_{\mu_{1} \ldots \mu_{J}, \alpha^{\prime}}^{(J-1)}\left(k^{\prime}\right) \psi_{n}^{(1, J-1, J)}\left(k^{\prime 2}\right)+B_{j} X_{\mu_{1} \ldots \mu_{J} \alpha^{\prime}}^{(J+1)}\left(k^{\prime}\right) \psi_{n}^{(1, J+1, J)}\left(k^{\prime 2}\right)\right),
\end{gathered}
$$

and

$$
\begin{gathered}
\left(s-M^{2}\right) Z_{\mu_{1} \ldots \mu_{J}, \beta}^{(J-1)}(k) S p\left[\gamma_{\alpha}^{\perp}\left(\widehat{k}_{1}+m\right) \gamma_{\beta}^{\perp}\left(-\widehat{k}_{2}+m\right)\right] \times \\
\times\left(A_{j} Z_{\mu_{1} \ldots \mu_{J}, \alpha}^{(J-1)}(k) \psi_{n}^{(1, J-1, J)}\left(k^{2}\right)+B_{j} X_{\mu_{1} \ldots \mu_{J} \alpha}^{(J+1)}(k) \psi_{n}^{(1, J+1, J)}\left(k^{2}\right)\right)= \\
=Z_{\mu_{1} \ldots \mu_{J}, \beta^{\prime}}^{(J-1)}(k) \sum_{c} S p\left[\widehat{F}_{c}\left(\widehat{k}_{1}+m\right) \gamma_{\beta^{\prime}}^{\perp}\left(-\widehat{k}_{2}+m\right)\right] \times \\
\times \int \frac{d^{3} k^{\prime}}{(2 \pi)^{3} k_{0}^{\prime}} V_{c}\left(s, s^{\prime},\left(k k^{\prime}\right)\right) S p\left[\gamma_{\alpha^{\prime}}^{\perp}\left(\widehat{k}_{1}^{\prime}+m^{\prime}\right) \widehat{F}_{c}\left(-\widehat{k}_{2}^{\prime}+m^{\prime}\right)\right] \times \\
\times\left(A_{j} Z_{\mu_{1} \ldots \mu_{J}, \alpha^{\prime}}^{(J-1)}\left(k^{\prime}\right) \psi_{n}^{(1, J-1, J)}\left(k^{\prime 2}\right)+B_{j} X_{\mu_{1} \ldots \mu_{J} \alpha^{\prime}}^{(J+1)}\left(k^{\prime}\right) \psi_{n}^{(1, J+1, J)}\left(k^{\prime 2}\right)\right) .
\end{gathered}
$$

First, let us consider (123); in the left-hand side of (123) one has two convolutions:

$$
\begin{gathered}
X_{\mu_{1} \ldots \mu_{J} \beta}^{(J+1)}(k) S p\left[\gamma_{\alpha}^{\perp}\left(\widehat{k}_{1}+m\right) \gamma_{\beta}^{\perp}\left(-\widehat{k}_{2}+m\right)\right] X_{\mu_{1} \ldots \mu_{J} \alpha}^{(J+1)}(k)= \\
=2 \alpha(J) k^{2(J+1)}\left[\frac{2 J+1}{J+1} s+4 k^{2}\right], \\
X_{\mu_{1} \ldots \mu_{J} \beta}^{(J+1)}(k) S p\left[\gamma_{\alpha}^{\perp}\left(\widehat{k}_{1}+m\right) \gamma_{\beta}^{\perp}\left(-\widehat{k}_{2}+m\right)\right] Z_{\mu_{1} \ldots \mu_{J}, \alpha}^{(J-1)}(k)= \\
=8 \alpha(J) k^{2(J+1)} .
\end{gathered}
$$

The left-hand side of (124) contains also two convolutions:

$$
\begin{gathered}
Z_{\mu_{1} \ldots \mu_{J}, \beta}^{(J-1)}(k) S p\left[\gamma_{\alpha}^{\perp}\left(\widehat{k}_{1}+m\right) \gamma_{\beta}^{\perp}\left(-\widehat{k}_{2}+m\right)\right] X_{\mu_{1} \ldots \mu_{J} \alpha}^{(J+1)}(k)= \\
=8 \alpha(J) k^{2(J+1)}, \\
Z_{\mu_{1} \ldots \mu_{J}, \beta}^{(J-1)}(k) S p\left[\gamma_{\alpha}^{\perp}\left(\widehat{k}_{1}+m\right) \gamma_{\beta}^{\perp}\left(-\widehat{k}_{2}+m\right)\right] Z_{\mu_{1} \ldots \mu_{J}, \alpha}^{(J-1)}(k)= \\
=2 \alpha(J) k^{2(J-1)}\left[\frac{2 J+1}{J} s+4 k^{2}\right] .
\end{gathered}
$$


The right-hand side of Eqs. (123) and (124) is determined by convolutions of the trace factor $B_{\beta^{\prime} \alpha^{\prime}}\left(s, s^{\prime},\left(k k^{\prime}\right)\right)$, see Eqs. (113) and (114), with angular momentum wave functions; corresponding formulae are presented in Appendix C. Following them, one has for the right-hand side of (123):

$$
\begin{gathered}
X_{\mu_{1} \ldots \mu_{J} \beta^{\prime}}^{(J+1)}(k) B_{\beta^{\prime} \alpha^{\prime}}\left(s, s^{\prime},\left(k k^{\prime}\right)\right) X_{\mu_{1} \ldots \mu_{J} \alpha^{\prime}}^{(J+1)}\left(k^{\prime}\right)=4 \alpha(J)\left(\sqrt{k^{2}} \sqrt{k^{\prime 2}}\right)^{J+1} \times \\
\times\left(\left[\frac{2 J+1}{J+1} \sqrt{s s^{\prime}}\left(\sqrt{s s^{\prime}} V_{V}\left(s, s^{\prime},\left(k k^{\prime}\right)\right)+8 m m^{\prime} V_{T}\left(s, s^{\prime},\left(k k^{\prime}\right)\right)\right)+\right.\right. \\
\left.+4 s^{\prime} k^{2} V_{V}\left(s, s^{\prime},\left(k k^{\prime}\right)\right)+4 s k^{\prime 2} V_{V}\left(s, s^{\prime}\right)+16 \frac{J+1}{2 J+1} k^{2} k^{\prime 2} V_{V}\left(s, s^{\prime},\left(k k^{\prime}\right)\right)\right] \times \\
\times P_{J+1}(z)+\left[16 m m^{\prime} V_{S}\left(s, s^{\prime},\left(k k^{\prime}\right)\right)+4 \frac{J}{J+1} \sqrt{s s^{\prime}} V_{A}\left(s, s^{\prime},\left(k k^{\prime}\right)\right)\right] \times \\
\left.\times \sqrt{k^{2}} \sqrt{k^{\prime 2}} P_{J}(z)+16 \frac{J}{2 J+1} k^{2} k^{\prime 2} V_{V}\left(s, s^{\prime},\left(k k^{\prime}\right)\right) P_{J-1}(z)\right)
\end{gathered}
$$

and

$$
\begin{gathered}
X_{\mu_{1} \ldots \mu_{J} \beta^{\prime}}^{(J+1)}(k) B_{\beta^{\prime} \alpha^{\prime}}\left(s, s^{\prime},\left(k k^{\prime}\right)\right) Z_{\mu_{1} \ldots \mu_{J}, \alpha^{\prime}}^{(J-1)}\left(k^{\prime}\right)= \\
=16 \alpha(J) k^{2}\left(\sqrt{k^{2}} \sqrt{k^{\prime 2}}\right)^{J-1}\left(\left[s+4 \frac{J+1}{2 J+1} k^{2}\right] k^{\prime 2} P_{J+1}(z) V_{V}\left(s, s^{\prime},\left(k k^{\prime}\right)\right)+\right. \\
+\left[-\sqrt{s s^{\prime}} V_{A}\left(s, s^{\prime},\left(k k^{\prime}\right)\right)+4 m m^{\prime} V_{S}\left(s, s^{\prime},\left(k k^{\prime}\right)\right)\right] \sqrt{k^{2}} \sqrt{k^{\prime 2}} P_{J}(z)+ \\
\left.+\left[s^{\prime}+4 \frac{J}{2 J+1} k^{2}\right] k^{2} P_{J-1}(z) V_{V}\left(s, s^{\prime},\left(k k^{\prime}\right)\right)\right) .
\end{gathered}
$$

For the right-hand side of (124) one has:

$$
\begin{gathered}
Z_{\mu_{1} \ldots \mu_{J}, \beta^{\prime}}^{(J-1)}(k) B_{\beta^{\prime} \alpha^{\prime}}\left(s, s^{\prime},\left(k k^{\prime}\right)\right) X_{\mu_{1} \ldots \mu_{J} \alpha^{\prime}}^{(J+1)}\left(k^{\prime}\right)= \\
=16 \alpha(J) k^{\prime 2}\left(\sqrt{k^{2}} \sqrt{k^{\prime 2}}\right)^{J-1}\left(\left[s^{\prime}+4 \frac{J+1}{2 J+1} k^{\prime 2}\right] k^{2} P_{J+1}(z) V_{V}\left(s, s^{\prime},\left(k k^{\prime}\right)\right)+\right. \\
+\left[-\sqrt{s s^{\prime}} V_{A}\left(s, s^{\prime},\left(k k^{\prime}\right)\right)+4 m m^{\prime} V_{S}\left(s, s^{\prime},\left(k k^{\prime}\right)\right)\right] \sqrt{k^{2}} \sqrt{k^{\prime 2}} P_{J}(z)+ \\
\left.+\left[s+4 \frac{J}{2 J+1} k^{2}\right] k^{\prime 2} P_{J-1}(z) V_{V}\left(s, s^{\prime},\left(k k^{\prime}\right)\right)\right)
\end{gathered}
$$

and

$$
\begin{gathered}
Z_{\mu_{1} \ldots \mu_{J}, \beta^{\prime}}^{(J-1)}(k) B_{\beta^{\prime} \alpha^{\prime}}\left(s, s^{\prime},\left(k k^{\prime}\right)\right) Z_{\mu_{1} \ldots \mu_{J}, \alpha^{\prime}}^{(J-1)}\left(k^{\prime}\right)=4 \alpha(J)\left(\sqrt{k^{2}} \sqrt{k^{\prime 2}}\right)^{J-1} \times \\
\times\left(16 \frac{J+1}{2 J+1} k^{2} k^{\prime 2} P_{J+1}(z) V_{V}\left(s, s^{\prime},\left(k k^{\prime}\right)\right)+\right.
\end{gathered}
$$




$$
\begin{gathered}
+\left[16 m m^{\prime} V_{S}\left(s, s^{\prime},\left(k k^{\prime}\right)\right)+4 \sqrt{s s^{\prime}} \frac{J+1}{J} V_{A}\left(s, s^{\prime},\left(k k^{\prime}\right)\right)\right] \sqrt{k^{2}} \sqrt{k^{\prime 2}} P_{J}(z)+ \\
+\left[\frac{2 J+1}{J} \sqrt{s s^{\prime}}\left(\sqrt{s s^{\prime}} V_{V}\left(s, s^{\prime},\left(k k^{\prime}\right)\right)+8 m m^{\prime} V_{T}\left(s, s^{\prime},\left(k k^{\prime}\right)\right)\right)+\right. \\
+4 s^{\prime} k^{2} V_{V}\left(s, s^{\prime},\left(k k^{\prime}\right)\right)+4 s k^{\prime 2} V_{V}\left(s, s^{\prime},\left(k k^{\prime}\right)\right)+ \\
\left.\left.+16 \frac{J}{2 J+1} k^{2} k^{\prime 2} V_{V}\left(s, s^{\prime},\left(k k^{\prime}\right)\right)\right] P_{J-1}(z)\right) .
\end{gathered}
$$

In the right-hand sides of Eqs. (123) and (124), we expand the interaction blocks in the Legendre polynomial series (94) and integrate over angle variables $\int_{-1}^{1} d z / 2$. As a result, Eq. (123) reads:

$$
\begin{gathered}
\left(s-M^{2}\right)\left[4 \psi_{n}^{(1, J-1, J)}\left(k^{2}\right) A_{j}+\left(\frac{2 J+1}{J+1} s+4 k^{2}\right) \psi_{n}^{(1, J+1, J)}\left(k^{2}\right) B_{j}\right]= \\
=\int_{4 m^{\prime 2}}^{\infty} \frac{d s^{\prime}}{\pi} \rho\left(s^{\prime}\right) 8\left(-k^{\prime 2}\right)^{J-1} \psi_{n}^{(1, J-1, J)}\left(k^{\prime 2}\right) A_{j} \times \\
\times\left[\xi(J+1)\left(s+4 \frac{J+1}{2 J+1} k^{2}\right) k^{\prime 4} \widetilde{V}_{V}^{(J+1)}\left(s, s^{\prime}\right)+\xi(J) \sqrt{s s^{\prime}} k^{\prime 2} \widetilde{V}_{A}^{(J)}\left(s, s^{\prime}\right)-\right. \\
\left.-4 m m^{\prime} k^{\prime 2} \xi(J) \widetilde{V}_{S}^{(J)}\left(s, s^{\prime}\right)+\xi(J-1)\left(s^{\prime}+4 \frac{J}{2 J+1} k^{\prime 2}\right) \widetilde{V}_{V}^{(J-1)}\left(s, s^{\prime}\right)\right]+ \\
+\int_{4 m^{\prime 2}}^{\infty} \frac{d s^{\prime}}{\pi} \rho\left(s^{\prime}\right) 2\left(-k^{\prime 2}\right)^{J+1} \psi_{n}^{(1, J+1, J)}\left(k^{\prime 2}\right) B_{j} \times \\
\times\left[8 m m^{\prime} \frac{j J+1}{J+1} \xi(J+1) \sqrt{s s^{\prime}} \widetilde{V}_{T}^{(J+1)}\left(s, s^{\prime}\right)+\right. \\
+16 m m^{\prime} \xi(J) \widetilde{V}_{S}^{(J)}\left(s, s^{\prime}\right)-4 \frac{J}{J+1} \xi(J) \sqrt{s s^{\prime}} \widetilde{V}_{A}^{(J)}\left(s, s^{\prime}\right)+ \\
+\xi(J+1)\left(\frac{2 J+1}{J+1} s s^{\prime}+4 s^{\prime} k^{2}+4 s k^{\prime 2}+16 \frac{J+1}{2 J+1} k^{2} k^{\prime 2}\right) \widetilde{V}_{V}^{(J+1)}\left(s, s^{\prime}\right)-
\end{gathered}
$$

The second equation, (124), reads:

$$
\left(s-M^{2}\right)\left[\left(\frac{2 J+1}{J} s+4 k^{2}\right) \psi_{n}^{(1, J-1, J)}\left(k^{2}\right) A_{j}+4 k^{4} \psi_{n}^{(1, J+1, J)}\left(k^{2}\right) B_{j}\right]=
$$




$$
\begin{gathered}
=\int_{4 m^{\prime 2}}^{\infty} \frac{d s^{\prime}}{\pi} \rho\left(s^{\prime}\right) 2\left(-k^{\prime 2}\right)^{J-1} \psi_{n}^{(1, J-1, J)}\left(k^{\prime 2}\right) A_{j} \times \\
\times\left[8 m m^{\prime} \frac{2 J+1}{J} \xi(J-1) \sqrt{s s^{\prime}} \widetilde{V}_{T}^{(J-1)}\left(s, s^{\prime}\right)+\right. \\
+\xi(J-1)\left(\frac{2 J+1}{J} s s^{\prime}+4 s^{\prime} k^{2}+4 s k^{\prime 2}+16 \frac{J}{2 J+1} k^{2} k^{\prime 2}\right) \widetilde{V}_{V}^{(J-1)}\left(s, s^{\prime}\right)- \\
-16 m m^{\prime} \xi(J) k^{2} k^{\prime 2} \widetilde{V}_{S}^{(J)}\left(s, s^{\prime}\right)-4 \frac{J+1}{J} \xi(J) \sqrt{s s^{\prime}} k^{2} k^{\prime 2} \widetilde{V}_{A}^{(J)}\left(s, s^{\prime}\right)+ \\
\left.+16 \frac{J+1}{2 J+1} \xi(J+1) k^{4} k^{\prime 4} \widetilde{V}_{V}^{(J+1)}\left(s, s^{\prime}\right)\right]+ \\
+\int_{4 m^{\prime 2}}^{\infty} \frac{d s^{\prime}}{\pi} \rho\left(s^{\prime}\right) 8\left(-k^{\prime 2}\right)^{J+1} \psi_{n}^{(1, J+1, J)}\left(k^{\prime 2}\right) B_{j} \times \\
\times\left[\xi(J+1)\left(s^{\prime}+4 \frac{J+1}{2 J+1} k^{\prime 2}\right) k^{4} \widetilde{V}_{V}^{(J+1)}\left(s, s^{\prime}\right)+\xi(J) \sqrt{s s^{\prime}} k^{2} \widetilde{V}_{A}^{(J)}\left(s, s^{\prime}\right)-\right. \\
\left.-4 m m^{\prime} \xi(J) k^{2} \widetilde{V}_{S}^{(J)}\left(s, s^{\prime}\right)+\xi(J-1)\left(s+4 \frac{J}{2 J+1} k^{2}\right) \widetilde{V}_{V}^{(J-1)}\left(s, s^{\prime}\right)\right]
\end{gathered}
$$

Normalization and orthoganality conditions determined by Eq. (87) are as follows:

$$
\begin{gathered}
\int_{4 m^{2}}^{\infty} \frac{d s}{\pi} \rho(s)\left[A_{j}^{2}\left(\psi_{n}^{(1, J-1, J)}\left(k^{2}\right)\right)^{2} 2 \alpha(J)\left(-k^{2}\right)^{(J-1)}\left(\frac{2 J+1}{J} s+4 k^{2}\right)+\right. \\
+2 A_{j} B_{j} \psi_{n}^{(1, J-1, J)}\left(k^{2}\right) \psi_{n}^{(1, J+1, J)}\left(k^{2}\right) 8 \alpha(J)\left(-k^{2}\right)^{(J+1)}+ \\
\left.+B_{j}^{2}\left(\psi_{n}^{(1, J+1, J)}\left(k^{2}\right)\right)^{2} 2 \alpha(J)\left(-k^{2}\right)^{(J+1)}\left(\frac{2 J+1}{J+1} s+4 k^{2}\right)\right]=1, \quad j=1,2,
\end{gathered}
$$

and

$$
\begin{gathered}
\int_{4 m^{2}}^{\infty} \frac{d s}{\pi} \rho(s)\left[A_{1} A_{2}\left(\psi_{n}^{(1, J-1, J)}\left(k^{2}\right)\right)^{2} 2 \alpha(J)\left(-k^{2}\right)^{(J-1)}\left(\frac{2 J+1}{J} s+4 k^{2}\right)+\right. \\
+\left(A_{1} B_{2}+A_{2} B_{1}\right) \psi_{n}^{(1, J-1, J)}\left(k^{2}\right) \psi_{n}^{(1, J+1, J)}\left(k^{2}\right) 8 \alpha(J)\left(-k^{2}\right)^{(J+1)}+ \\
\left.+B_{1} B_{2}\left(\psi_{n}^{(1, J+1, J)}\left(k^{2}\right)\right)^{2} 2 \alpha(J)\left(-k^{2}\right)^{(J+1)}\left(\frac{2 J+1}{J+1} s+4 k^{2}\right)\right]=0 .
\end{gathered}
$$

Let us emphasize again: all the above equations are written for the case $J>0$. 


\section{Equation for the $a_{0}\left(M^{2}, n\right)$-trajectory}

For the $(S=1, L=1, J=0)$ state, we have only one level $L=J+1$; the wave function of this state obeys the equation:

$$
\begin{gathered}
\left(s-M^{2}\right)\left(s+4 k^{2}\right) \psi_{a_{0}, n}^{(1,1,0)}(s)=-\int_{4 m^{\prime 2}}^{\infty} \frac{d s^{\prime}}{\pi} \rho\left(s^{\prime}\right) 2 k^{\prime 2} \psi_{a_{0}, n}^{(1,1,0)}\left(s^{\prime}\right) \times \\
\times\left[\frac{8}{3} m^{2} \sqrt{s s^{\prime}} \widetilde{V}_{T}^{(1)}\left(s, s^{\prime}\right)+\frac{1}{3}\left(s s^{\prime}+4 s^{\prime} k^{2}+4 s k^{\prime 2}+16 k^{2} k^{\prime 2}\right) \widetilde{V}_{V}^{(1)}\left(s, s^{\prime}\right)-\right. \\
\left.-16 m^{2} \widetilde{V}_{S}^{(0)}\left(s, s^{\prime}\right)\right] .
\end{gathered}
$$

According to [15], the following states are located on the $a_{0}\left(M^{2}, n\right)$-trajectory:

$a_{0}(980)$ with $n=1, a_{0}(1520)$ with $n=2, a_{0}(1830)$ with $n=3, a_{0}(2120)$ with $n=3$, and so on.

The normalization reads:

$$
\int_{4 m^{2}}^{\infty} \frac{d s}{\pi} \rho(s)\left(\psi_{a_{0}, n}^{(1,1,0)}(s)\right)^{2} 2\left(-k^{2}\right)\left(s+4 k^{2}\right)=1 .
$$

\section{Equation for the $f_{0}\left(M^{2}, n\right)$-trajectory}

The $f_{0}$-states have two flavor components, $n \bar{n}$ and $s \bar{s}$, correspondingly we have two equations for two wave functions:

$$
\begin{gathered}
\left(s-M^{2}\right)\left(s+4 k^{2}\right) \psi_{a_{0}(n \bar{n}), n}^{(1,1,0)}(s) \cos \Theta_{n}= \\
=-\int_{4 m^{2}}^{\infty} \frac{d s^{\prime}}{\pi} \rho\left(s^{\prime}\right) 2 k^{\prime 2} \psi_{a_{0}(n \bar{n}), n}^{(1,1,0)}\left(s^{\prime}\right) \cos \Theta_{n}\left[\frac{8}{3} m^{2} \sqrt{s s^{\prime}} \widetilde{V}_{(n \bar{n} \rightarrow n \bar{n}), T}^{(1)}\left(s, s^{\prime}\right)+\right. \\
+\frac{1}{3}\left(s s^{\prime}+4 s^{\prime} k^{2}+4 s k^{\prime 2}+16 k^{2} k^{\prime 2}\right) \widetilde{V}_{(n \bar{n} \rightarrow n \bar{n}), V}^{(1)}\left(s, s^{\prime}\right)- \\
\left.-16 m^{2} \widetilde{V}_{(n \bar{n} \rightarrow n \bar{n}), S}^{(0)}\left(s, s^{\prime}\right)\right]-\int_{4 m_{s}^{2}}^{\infty} \frac{d s^{\prime}}{\pi} \rho_{s}\left(s^{\prime}\right) 2 k_{s}^{\prime 2} \psi_{a_{0}(s \bar{s}), n}^{(1,1,0)}\left(s^{\prime}\right) \sin \Theta_{n} \times \\
\times\left[\frac{8}{3} m m_{s} \sqrt{s s^{\prime}} \widetilde{V}_{(s \bar{s} \rightarrow n \bar{n}), T}^{(1)}\left(s, s^{\prime}\right)+\frac{1}{3}\left(s s^{\prime}+4 s^{\prime} k^{2}+4 s k_{s}^{\prime 2}+16 k^{2} k_{s}^{\prime 2}\right) \times\right. \\
\left.\times \widetilde{V}_{(s \bar{s} \rightarrow n \bar{n}), V}^{(1)}\left(s, s^{\prime}\right)-16 m m_{s} \widetilde{V}_{(s \bar{s} \rightarrow n \bar{n}), S}^{(0)}\left(s, s^{\prime}\right)\right]
\end{gathered}
$$

and

$$
\left(s-M^{2}\right)\left(s+4 k^{2}\right) \psi_{a_{0}(s \bar{s}), n}^{(1,1,0)}(s) \sin \Theta_{n}=
$$




$$
\begin{gathered}
=-\int_{4 m^{2}}^{\infty} \frac{d s^{\prime}}{\pi} \rho\left(s^{\prime}\right) 2 k^{\prime 2} \psi_{a_{0}(n \bar{n}), n}^{(1,1,0)}\left(s^{\prime}\right) \cos \Theta_{n}\left[\frac{8}{3} m m_{s} \sqrt{s s^{\prime}} \widetilde{V}_{(n \bar{n} \rightarrow s \bar{s}), T}^{(1)}\left(s, s^{\prime}\right)+\right. \\
+\frac{1}{3}\left(s s^{\prime}+4 s^{\prime} k_{s}^{2}+4 s k^{\prime 2}+16 k_{s}^{2} k^{\prime 2}\right) \widetilde{V}_{(n \bar{n} \rightarrow s \bar{s}), V}^{(1)}\left(s, s^{\prime}\right)- \\
\left.-16 m m_{s} \widetilde{V}_{(n \bar{n} \rightarrow s \bar{s}), S}^{(0)}\left(s, s^{\prime}\right)\right]-\int_{4 m_{s}^{2}}^{\infty} \frac{d s^{\prime}}{\pi} \rho_{s}\left(s^{\prime}\right) 2 k_{s}^{\prime 2} \psi_{a_{0}(s \bar{s}), n}^{(1,1,0)}\left(s^{\prime}\right) \sin \Theta_{n} \times \\
\times\left[\frac{8}{3} m_{s}^{2} \sqrt{s s^{\prime}} \widetilde{V}_{(s \bar{s} \rightarrow s \bar{s}), T}^{(1)}\left(s, s^{\prime}\right)+\frac{1}{3}\left(s s^{\prime}+4 s^{\prime} k_{s}^{2}+4 s k_{s}^{\prime 2}+16 k_{s}^{2} k_{s}^{\prime 2}\right) \times\right. \\
\left.\times \widetilde{V}_{(s \bar{s} \rightarrow s \bar{s}), V}^{(1)}\left(s, s^{\prime}\right)-16 m_{s}^{2} \widetilde{V}_{(s \bar{s} \rightarrow s \bar{s}), S}^{(0)}\left(s, s^{\prime}\right)\right] .
\end{gathered}
$$

The following states are located on two $f_{0}\left(M^{2}, n\right)$-trajectories [15]:

1) $f_{0}(980)$ with $n=1, f_{0}(1500)$ with $n=2, f_{0}(2005)$ with $n=3, f_{0}(2240)$ with $n=4$, and so on,

2) $f_{0}(1300)$ with $n=1, f_{0}(1750)$ with $n=2, f_{0}(2105)$ with $n=3, f_{0}(2330)$ with $n=4$, and so on.

\section{Equation for the $\rho\left(M^{2}, n\right)$-trajectory}

Two equations read:

$$
\begin{gathered}
\left(s-M^{2}\right)\left[4 \psi_{\rho, n}^{(1,0,1)}(s) A_{j}+\left(\frac{3}{2} s+4 k^{2}\right) \psi_{\rho, n}^{(1,2,1)}(s) B_{j}\right]= \\
=\int_{4 m^{2}}^{\infty} \frac{d s^{\prime}}{\pi} \rho\left(s^{\prime}\right) 8 \psi_{\rho, n}^{(1,0,1)}\left(s^{\prime}\right) A_{j} \times \\
\times\left[\frac{3}{10}\left(s+\frac{8}{3} k^{2}\right) k^{\prime 4} \widetilde{V}_{V}^{(2)}\left(s, s^{\prime}\right)+\frac{1}{3} \sqrt{s s^{\prime}} k^{\prime 2} \widetilde{V}_{A}^{(1)}\left(s, s^{\prime}\right)-\right. \\
\left.-\frac{4}{3} m^{2} k^{\prime 2} \widetilde{V}_{S}^{(1)}\left(s, s^{\prime}\right)+\left(s^{\prime}+\frac{4}{3} k^{\prime 2}\right) \widetilde{V}_{V}^{(0)}\left(s, s^{\prime}\right)\right]+ \\
+\int_{4 m^{2}}^{\infty} \frac{d s^{\prime}}{\pi} \rho\left(s^{\prime}\right) 2 k^{\prime 4} \psi_{\rho, n}^{(1,2,1)}\left(s^{\prime}\right) B_{j} \times \\
\times\left[m^{2} \frac{18}{5} \sqrt{s s^{\prime}} \widetilde{V}_{T}^{(2)}\left(s, s^{\prime}\right)+\frac{3}{10}\left(\frac{3}{2} s s^{\prime}+4 s^{\prime} k^{2}+4 s k^{\prime 2}+\frac{32}{3} k^{2} k^{\prime 2}\right) \widetilde{V}_{V}^{(2)}\left(s, s^{\prime}\right)-\right. \\
\left.-m^{2} \frac{16}{3} \widetilde{V}_{S}^{(1)}\left(s, s^{\prime}\right)-\frac{2}{3} \sqrt{s s^{\prime}} \widetilde{V}_{A}^{(1)}\left(s, s^{\prime}\right)+\frac{16}{3} \widetilde{V}_{V}^{(0)}\left(s, s^{\prime}\right)\right]
\end{gathered}
$$

and 


$$
\begin{gathered}
\left(s-M^{2}\right)\left[\left(3 s+4 k^{2}\right) \psi_{\rho, n}^{(1,0,1)}(s) A_{j}+4 k^{4} \psi_{\rho, n}^{(1,2,1)}(s) B_{j}\right]= \\
=\int_{4 m^{2}}^{\infty} \frac{d s^{\prime}}{\pi} \rho\left(s^{\prime}\right) 2 \psi_{\rho, n}^{(1,0,1)}\left(s^{\prime}\right) A_{j} \times \\
\times\left[24 m^{2} \sqrt{s s^{\prime}} \widetilde{V}_{T}^{(0)}\left(s, s^{\prime}\right)+\left(3 s s^{\prime}+4 s^{\prime} k^{2}+4 s k^{\prime 2}+\frac{16}{3} k^{2} k^{\prime 2}\right) \widetilde{V}_{V}^{(0)}\left(s, s^{\prime}\right)-\right. \\
\left.-m^{2} \frac{16}{3} k^{2} k^{\prime 2} \widetilde{V}_{S}^{(1)}\left(s, s^{\prime}\right)-\frac{8}{3} \sqrt{s s^{\prime}} k^{2} k^{\prime 2} \widetilde{V}_{A}^{(1)}\left(s, s^{\prime}\right)+\frac{16}{5} k^{4} k^{\prime 4} \widetilde{V}_{V}^{(2)}\left(s, s^{\prime}\right)\right]+ \\
+\int_{4 m^{2}}^{\infty} \frac{d s^{\prime}}{\pi} \rho\left(s^{\prime}\right) 8 k^{\prime 4} \psi_{\rho, n}^{(1,2,1)}\left(s^{\prime}\right) B_{j} \times \\
\times\left[\frac{3}{10}\left(s^{\prime}+\frac{8}{3} k^{\prime 2}\right) k^{4} \widetilde{V}_{V}^{(2)}\left(s, s^{\prime}\right)+\frac{1}{3} \sqrt{s s^{\prime}} k^{2} \widetilde{V}_{A}^{(1)}\left(s, s^{\prime}\right)-\right. \\
\left.-m^{2} \frac{4}{3} k^{2} \widetilde{V}_{S}^{(1)}\left(s, s^{\prime}\right)+\left(s+\frac{4}{3} k^{2}\right) \widetilde{V}_{V}^{(0)}\left(s, s^{\prime}\right)\right]
\end{gathered}
$$

The following states are located on the $\rho\left(M^{2}, n\right)$-trajectories [15]:

1) $\rho(770)$ with $n=1, \rho(1450)$ with $n=2, \rho(1830)$ with $n=3, \rho(2110)$ with $n=4$, and so on,

2) $\rho(1700)$ with $n=1, \rho(1990)$ with $n=2, \rho(2285)$ with $n=3$, and so on.

Normalization and orthogonality conditions are as follows:

$$
\begin{aligned}
& \int_{4 m^{2}}^{\infty} \frac{d s}{\pi} \rho(s)\left[A_{j}^{2}\left(\psi_{\rho, n}^{(1,0,1)}(s)\right)^{2} 2\left(3 s+4 k^{2}\right)+\right. \\
& +2 A_{j} B_{j} \psi_{\rho, n}^{(1,0,1)}(s) \psi_{\rho, n}^{(1,2,1)}(s) 8 k^{4}+ \\
& \left.+B_{j}^{2}\left(\psi_{\rho, n}^{(1,2,1)}(s)\right)^{2} 2 k^{4}\left(\frac{3}{2} s+4 k^{2}\right)\right]=1, \quad j=1,2,
\end{aligned}
$$

and

$$
\begin{gathered}
\int_{4 m^{2}}^{\infty} \frac{d s}{\pi} \rho(s)\left[A_{1} A_{2}\left(\psi_{\rho, n}^{(1,0,1)}(s)\right)^{2} 2\left(3 s+4 k^{2}\right)+\right. \\
+\left(A_{1} B_{2}+A_{2} B_{1}\right) \psi_{\rho, n}^{(1,0,1)}(s) \psi_{\rho, n}^{(1,2,1)}(s) 8 k^{4}+ \\
\left.+B_{1} B_{2}\left(\psi_{\rho, n}^{(1,2,1)}(s)\right)^{2} 2 k^{4}\left(\frac{3}{2} s+4 k^{2}\right)\right]=0 .
\end{gathered}
$$

\section{ACKNOWLEDGEMENTS}

We are grateful to D.V.Bugg, L.G.Dakhno, D.I.Melikhov, V.A.Nikonov, and H.R.Petry for helpful and stimulating discussions. The work was supported by RFBR grants 0102-17861 and 0102-17152. One of us (VNM) thanks for support INTAS, project 2000-587, and Prof. H.R.Petry for the hospitality in ITKP(Bonn). 


\section{APPENDIX A: APPENDIX: ANGULAR-MOMENTUM OPERATORS}

Here we present the angular momentum operator $X_{\mu_{1} \mu_{2} \ldots \mu_{L-1} \mu_{L}}^{(L)}(k)$ and briefly recall its properties; a full presentation of the angular-momentum operators can be found in [9].

The operator $X_{\mu_{1} \mu_{2} \ldots \mu_{L-1} \mu_{L}}^{(L)}(k)$ is constructed by using relative momentum of mesons in the space orthogonal to the total momentum $P$ :

$$
k_{\mu}^{\perp}=k_{\nu} g_{\nu \mu}^{\perp}, \quad g_{\nu \mu}^{\perp}=g_{\nu \mu}-\frac{P_{\nu} P_{\mu}}{s}, \quad g_{\mu \nu}=(1,-1,-1,-1) .
$$

In the center-of-mass system, where $P=\left(P_{0}, \vec{P}\right)=(\sqrt{s}, 0)$, the vector $k^{\perp}$ is space-like: $k^{\perp}=(0, \vec{k})$. We determine the operator $X_{\mu_{1} \mu_{2} \ldots \mu_{L-1} \mu_{L}}^{(L)}(k)$ as symmetrical and traceless. It is easy to construct it for the lowest values of $L=0,1,2,3$ :

$$
\begin{gathered}
X^{(0)}=1, \quad X_{\mu}^{(1)}=k_{\mu}^{\perp}, \quad X_{\mu_{1} \mu_{2}}^{(2)}=\frac{3}{2}\left(k_{\mu_{1}}^{\perp} k_{\mu_{2}}^{\perp}-\frac{1}{3}\left(k^{\perp}\right)^{2} g_{\mu_{1} \mu_{2}}^{\perp}\right), \\
X_{\mu_{1} \mu_{2} \mu_{3}}^{(3)}=\frac{5}{2}\left[k_{\mu_{1}}^{\perp} k_{\mu_{2}}^{\perp} k_{\mu_{3}}^{\perp}-\frac{\left(k^{\perp}\right)^{2}}{5}\left(g_{\mu_{1} \mu_{2}}^{\perp} k_{\mu_{3}}^{\perp}+g_{\mu_{1} \mu_{3}}^{\perp} k_{\mu_{2}}^{\perp}+g_{\mu_{2} \mu_{3}}^{\perp} k_{\mu_{1}}^{\perp}\right)\right] .
\end{gathered}
$$

Correspondingly, the generalization of $X_{\mu_{1} \ldots \mu_{L}}^{(L)}$ for $L>1$ reads:

$$
\begin{aligned}
X_{\mu_{1} \ldots \mu_{L}}^{(L)}= & k_{\alpha}^{\perp} Z_{\mu_{1} \ldots \mu_{L}, \alpha}^{(L-1)}, \\
Z_{\mu_{1} \ldots \mu_{L}, \alpha}^{(L-1)}= & \frac{2 L-1}{L^{2}}\left(\sum_{i=1}^{L} X_{\mu_{1} \ldots \mu_{i-1} \mu_{i+1} \ldots \mu_{L}}^{(L-1)} g_{\mu_{i} \alpha}^{\perp}-\right. \\
& \left.-\frac{2}{2 L-1} \sum_{\substack{i, j=1 \\
i<j}}^{L} g_{\mu_{i} \mu_{j}}^{\perp} X_{\mu_{1} \ldots \mu_{i-1} \mu_{i+1} \ldots \mu_{j-1} \mu_{j+1} \ldots \mu_{L} \alpha}^{(L-1)}\right) .
\end{aligned}
$$

It is seen that the operator $X_{\mu_{1} \mu_{2} \ldots \mu_{L-1} \mu_{L}}^{(L)}(k)$ constructed in accordance with (A.3) is symmetrical,

$$
X_{\mu_{1} \ldots \mu_{i} \ldots \mu_{j} \ldots \mu_{L}}^{(L)}=X_{\mu_{1} \ldots \mu_{j} \ldots \mu_{i} \ldots \mu_{L}}^{(L)},
$$

and it works in the space orthogonal to $P$ :

$$
P_{\mu_{i}} X_{\mu_{1} \ldots \mu_{i} \ldots \mu_{L}}^{(L)}=0
$$

The angular-momentum operator $X_{\mu_{1} \ldots \mu_{L}}^{(L)}$ is traceless over any two indices:

$$
g_{\mu_{i} \mu_{j}} X_{\mu_{1} \ldots \mu_{i} \ldots \mu_{j} \ldots \mu_{L}}^{(L)}=g_{\mu_{i} \mu_{j}}^{\perp} X_{\mu_{1} \ldots \mu_{i} \ldots \mu_{j} \ldots \mu_{L}}^{(L)}=0 .
$$

The tracelessness property given by (A.6) is obvious for the lowest-order operators entering (A.2), for example, $g_{\mu_{1} \mu_{2}}^{\perp} X_{\mu_{1} \mu_{2}}^{(2)}=0$ (recall that $g_{\mu_{1} \mu_{2}}^{\perp} g_{\mu_{1} \mu_{2}}^{\perp}=3$ ).

The convolution equality reads:

$$
X_{\mu_{1} \ldots \mu_{L}}^{(L)} k_{\mu_{L}}^{\perp}=\left(k^{\perp}\right)^{2} X_{\mu_{1} \ldots \mu_{L-1}}^{(L-1)}
$$

Using (A.7) we rewrite the recurrent equation (A.3) in the form:

$$
\begin{gathered}
X_{\mu_{1} \ldots \mu_{L}}^{(L)}= \\
=\frac{2 L-1}{L^{2}} \sum_{i=1}^{L} k_{\mu_{i}}^{\perp} X_{\mu_{1} \ldots \mu_{i-1} \mu_{i+1} \ldots \mu_{L}}^{(L-1)}-\frac{2\left(k^{\perp}\right)^{2}}{L^{2}} \sum_{\substack{i, j=1 \\
i<j}}^{L} g_{\mu_{i} \mu_{j}}^{\perp} X_{\mu_{1} \ldots \mu_{i-1} \mu_{i+1} \ldots \mu_{j-1} \mu_{j+1} \ldots \mu_{L}}^{(L-2)} .
\end{gathered}
$$


On the basis of this recurrent equation and taking into account the tracelessness of $X_{\mu_{1} \ldots \mu_{L}}^{(L)}$, one can write the normalization condition for the moment- $L$ operator as follows:

$$
X_{\mu_{1} \ldots \mu_{L}}^{(L)}(k) X_{\mu_{1} \ldots \mu_{L}}^{(L)}(k)=\alpha(L)\left(k^{\perp}\right)^{2 L} \quad, \alpha(L)=\prod_{l=1}^{L} \frac{2 l-1}{l}=\frac{(2 L-1) ! !}{L !} .
$$

The iteration of (A.8) gives us the following expression for the operator $X_{\mu_{1} \ldots \mu_{L}}^{(L)}$ :

$$
\begin{aligned}
& X_{\mu_{1} \ldots \mu_{L}}^{(L)}(k)=\frac{(2 L-1) ! !}{L !}\left[k_{\mu_{1}}^{\perp} k_{\mu_{2}}^{\perp} k_{\mu_{3}}^{\perp} k_{\mu_{4}}^{\perp} \ldots k_{\mu_{L}}^{\perp}-\right. \\
& -\frac{\left(k^{\perp}\right)^{2}}{2 L-1}\left(g_{\mu_{1} \mu_{2}}^{\perp} k_{\mu_{3}}^{\perp} k_{\mu_{4}}^{\perp} \ldots k_{\mu_{L}}^{\perp}+g_{\mu_{1} \mu_{3}}^{\perp} k_{\mu_{2}}^{\perp} k_{\mu_{4}}^{\perp} \ldots k_{\mu_{L}}^{\perp}+\ldots\right)+ \\
& +\frac{\left(k^{\perp}\right)^{4}}{(2 L-1)(2 L-3)}\left(g_{\mu_{1} \mu_{2}}^{\perp} g_{\mu_{3} \mu_{4}}^{\perp} k_{\mu_{5}}^{\perp} k_{\mu_{6}}^{\perp} \ldots k_{\mu_{L}}^{\perp}+\right. \\
& \left.\left.+g_{\mu_{1} \mu_{2}}^{\perp} g_{\mu_{3} \mu_{5}}^{\perp} k_{\mu_{4}}^{\perp} k_{\mu_{6}}^{\perp} \ldots k_{\mu_{L}}^{\perp}+\ldots\right)+\ldots\right] .
\end{aligned}
$$

One can introduce a projection operator $O_{\nu_{1} \ldots \nu_{L}}^{\mu_{1} \ldots \mu_{L}}$ for the partial wave with the angular momentum $L$. The operator is defined by the following relations:

$$
X_{\mu_{1} \ldots \mu_{L}}^{(L)}(k) O_{\nu_{1} \ldots \nu_{L}}^{\mu_{1} \ldots \mu_{L}}=X_{\nu_{1} \ldots \nu_{L}}^{(L)}(k), \quad O_{\alpha_{1} \ldots \alpha_{L}}^{\mu_{1} \ldots \mu_{L}} O_{\nu_{1} \ldots \nu_{L}}^{\alpha_{1} \ldots \alpha_{L}}=O_{\nu_{1} \ldots \nu_{L}}^{\mu_{1} \ldots \mu_{L}}
$$

For the sets of indices $\mu_{1} \ldots \mu_{L}$ and $\nu_{1} \ldots \nu_{L}$, the operator $O$ has all the properties of the operator $\hat{X}^{(L)}$ : it is symmetrical and traceless,

$$
O_{\nu_{1} \nu_{2} \ldots \nu_{L}}^{\mu_{1} \mu_{2} \ldots \mu_{L}}=O_{\nu_{1} \nu_{2} \ldots \nu_{L}}^{\mu_{2} \mu_{1} \ldots \mu_{L}}=O_{\nu_{2} \nu_{1} \ldots \nu_{L}}^{\mu_{1} \mu_{2} \ldots \mu_{L}}, \quad O_{\nu_{1} \nu_{2} \ldots \nu_{L}}^{\mu_{1} \mu_{1} \ldots \mu_{L}}=O_{\nu_{1} \nu_{1} \ldots \nu_{L}}^{\mu_{1} \mu_{2} \ldots \mu_{L}}=0
$$

The projection operator $O$ can be consructed as a product of the operators $X_{\mu_{1} \ldots \mu_{L}}^{(L)}(k) X_{\nu_{1} \ldots \nu_{L}}^{(L)}(k)$ integrated over angular variables of the momentum $k^{\perp}$, so we have a convolution of the $(2 L+1)$ dimensional vectors, which provide us with irreducible representation of Lorentz group in the $k^{\perp} /\left|k^{\perp}\right|$-space. So,

$$
\xi(L) O_{\nu_{1} \ldots \nu_{L}}^{\mu_{1} \ldots \mu_{L}}=\frac{1}{\left(k^{\perp}\right)^{2 L}} \int \frac{d \Omega}{4 \pi} X_{\mu_{1} \ldots \mu_{L}}^{(L)}(k) X_{\nu_{1} \ldots \nu_{L}}^{(L)}(k)
$$

where $\xi(L)$ is a normalization factor fixed below. Using the definition of the projection operator $O_{\nu_{1} \ldots \nu_{L}}^{\mu_{1} \ldots \mu_{L}}$ we have:

$$
k_{\mu_{1}} \ldots k_{\mu_{L}} O_{\nu_{1} \ldots \nu_{L}}^{\mu_{1} \ldots \mu_{L}}=\frac{1}{\alpha(L)} X_{\nu_{1} \ldots \nu_{L}}^{(L)}(k) .
$$

This equation represents the basic property of the operator: it projects any index- $L$ operator into partial-wave operator with the angular momentum $L$.

Multiplying the equation (A.13) by the product $X_{\mu_{1} \ldots \mu_{L}}^{(L)}(q) \cdot X_{\nu_{1} \ldots \nu_{L}}^{(L)}(q)$, we get:

$$
\xi(L) X_{\nu_{1} \ldots \nu_{L}}^{(L)}(q) X_{\nu_{1} \ldots \nu_{L}}^{(L)}(q)=\left(q^{\perp}\right)^{2 L} \alpha^{2}(L) \int_{-1}^{1} \frac{d z}{2} P_{L}^{2}(z)
$$

that gives the normalization constant in (A.13):

$$
\xi(L)=\frac{\alpha(L)}{2 L+1}=\frac{(2 L-1) ! !}{(2 L+1) \cdot L !} .
$$

Summation in the projection operator over upper and lower indices performed in (A.13) gives us the following reduction formula:

$$
O_{\nu_{1} \ldots \nu_{L-1} \mu_{L}}^{\mu_{1} \ldots \mu_{L-1} \mu_{L}}=\frac{2 L+1}{2 L-1} O_{\nu_{1} \ldots \nu_{L-1}}^{\mu_{1} \ldots \mu_{L-1}}
$$


Likewise, the summation over all indices gives us:

$$
O_{\mu_{1} \ldots \mu_{L}}^{\mu_{1} \ldots \mu_{L}}=2 L+1
$$

that can be proven using formula (A.13). On the basis of the equation (A.14), one gets

$$
X_{\mu_{1} \ldots \mu_{L-1} \mu_{L}}^{(L)} O_{\nu_{1} \ldots \nu_{L-1}}^{\mu_{1} \ldots \mu_{L-1}}=X_{\nu_{1} \ldots \nu_{L-1} \mu_{L}}^{(L)} .
$$

Generally, one can write:

$$
X_{\mu_{1} \ldots \mu_{i} \mu_{i+1} \ldots \mu_{L}}^{(L)} O_{\nu_{1} \ldots \nu_{i}}^{\mu_{1} \ldots \mu_{i}}=X_{\nu_{1} \ldots \nu_{i} \mu_{i+1} \mu_{L}}^{(L)}
$$

\section{APPENDIX B: APPENDIX: TRACES OF THE LOOP DIAGRAMS}

Here we present the traces which are used for the calculations of loop diagrams. Recall that in the spectral-integral representation, there is no energy conservation, $s \neq s^{\prime}$, where $P^{2}=s, P^{\prime 2}=s^{\prime}$, but all constituents are mass-on-shell:

$$
k_{1}^{2}=k_{2}^{2}=m^{2}, \quad k_{1}^{\prime 2}=k_{2}^{\prime 2}=m^{\prime 2} .
$$

The following notations are used for the quark momenta:

$$
\begin{aligned}
& k_{\nu}=\frac{1}{2}\left(k_{1}-k_{2}\right)_{\nu}, \quad k_{\nu}^{\prime}=\frac{1}{2}\left(k_{1}^{\prime}-k_{2}^{\prime}\right)_{\nu}, \\
& k_{\mu}^{\perp}=k_{\nu} g_{\nu \mu}^{\perp}=k_{\mu}, \quad k_{\mu}^{\prime \perp}=k_{\nu}^{\prime} g_{\nu \mu}^{\perp}=k_{\mu}^{\prime} .
\end{aligned}
$$

We follow the definition of matrices:

$$
\gamma_{5}=-i \gamma^{0} \gamma^{1} \gamma^{2} \gamma^{3}, \quad \sigma_{\mu \nu}=\frac{1}{2}\left[\gamma_{\mu} \gamma_{\nu}\right]
$$

1. Traces for the $S=0$ states

For the $S=0$ states we have the following non-zero traces:

$$
\begin{aligned}
& T_{P}^{\prime}=S p\left[i \gamma_{5}\left(k_{1}^{\prime}+m^{\prime}\right) \gamma_{5}\left(-k_{2}^{\prime}+m^{\prime}\right)\right]=2 i s^{\prime}, \\
& T_{A}^{\prime}=S p\left[i \gamma_{5}\left(k_{1}^{\prime}+m^{\prime}\right) i \gamma_{\mu} \gamma_{5}\left(-k_{2}^{\prime}+m^{\prime}\right)\right]=-4 m^{\prime} P_{\mu}^{\prime}, \\
& T_{T}^{\prime}=S p\left[i \gamma_{5}\left(k_{1}^{\prime}+m^{\prime}\right) i \sigma_{\mu \nu}\left(-k_{2}^{\prime}+m^{\prime}\right)\right]=-4 i \epsilon_{\mu \nu \alpha \beta} P_{\alpha}^{\prime} k_{\beta}^{\prime},
\end{aligned}
$$

and

$$
\begin{aligned}
& T_{P}=S p\left[i \gamma_{5}\left(-k_{2}+m\right) \gamma_{5}\left(k_{1}+m\right)\right]=2 i s \\
& T_{A}=S p\left[i \gamma_{5}\left(-k_{2}+m\right) i \gamma_{\mu} \gamma_{5}\left(k_{1}+m\right)\right]=4 m P_{\mu} \\
& T_{T}=S p\left[i \gamma_{5}\left(-k_{2}+m\right) i \sigma_{\mu \nu}\left(k_{1}+m\right)\right]=4 i \epsilon_{\mu \nu \alpha \beta} P_{\alpha} k_{\beta} .
\end{aligned}
$$

The convolutions of the traces $A_{P}=\left(T_{P} T_{P}^{\prime}\right), A_{A}=\left(T_{A} T_{A}^{\prime}\right)$,

$A_{T}=\left(T_{T} T_{T}^{\prime}\right)$ are equal to:

$$
\begin{aligned}
& A_{P}=-4 s s^{\prime} \\
& A_{A}=-16 m m^{\prime}\left(P P^{\prime}\right), \\
& A_{T}=-32\left(P P^{\prime}\right)\left(k k^{\prime}\right) .
\end{aligned}
$$




\section{Traces for the $S=1$ states}

The case of the $(S=1)$ states, the traces are equal to:

$$
\begin{aligned}
& T_{S}^{\prime}=S p\left[\gamma_{\alpha^{\prime}}^{\perp}\left(k_{1}^{\prime}+m^{\prime}\right) \cdot I \cdot\left(-k_{2}^{\prime}+m^{\prime}\right)\right]=8 m^{\prime} k_{\alpha^{\prime}}^{\prime}, \\
& T_{V}^{\prime}=S p\left[\gamma_{\alpha^{\prime}}^{\perp}\left(k_{1}^{\prime}+m^{\prime}\right) \gamma_{\mu}\left(-k_{2}^{\prime}+m^{\prime}\right)\right]=2\left(g_{\alpha^{\prime} \mu}^{\perp} s^{\prime}+4 k_{\alpha^{\prime}}^{\prime} k_{\mu}^{\prime}\right), \\
& T_{A}^{\prime}=S p\left[\gamma_{\alpha^{\prime}}^{\perp}\left(k_{1}^{\prime}+m^{\prime}\right) i \gamma_{\mu}^{\perp} \gamma_{5}\left(-k_{2}^{\prime}+m^{\prime}\right)\right]=4 \epsilon_{\alpha^{\prime} \mu \alpha \beta} k_{\alpha}^{\prime} P_{\beta}^{\prime}, \\
& T_{T}^{\prime}=S p\left[\gamma_{\alpha^{\prime}}^{\perp}\left(k_{1}^{\prime}+m^{\prime}\right) i \sigma_{\mu \nu}\left(-k_{2}^{\prime}+m^{\prime}\right)\right]=4 m^{\prime} i\left[g_{\alpha^{\prime} \nu}^{\perp} P_{\mu}^{\prime}-g_{\alpha^{\prime} \mu}^{\perp} P_{\nu}^{\prime}\right],
\end{aligned}
$$

and

$$
\begin{aligned}
& T_{S}=S p\left[\gamma_{\beta}^{\perp}\left(-k_{2}+m\right) \cdot \mathrm{I} \cdot\left(k_{1}+m\right)\right]=8 m k_{\beta}, \\
& T_{V}=S p\left[\gamma_{\beta}^{\perp}\left(-k_{2}+m\right) \gamma_{\mu}\left(k_{1}+m\right)\right]=2\left[g_{\mu \beta}^{\perp} s+4 k_{\beta} k_{\mu}\right], \\
& T_{A}=S p\left[\gamma_{\beta}^{\perp}\left(-k_{2}+m\right) i \gamma_{\mu} \gamma_{5}\left(k_{1}+m\right)\right]=-4 \epsilon_{\beta \mu \alpha^{\prime} \beta^{\prime}} k_{\alpha^{\prime}} P_{\beta^{\prime}}, \\
& T_{T}=S p\left[\gamma_{\beta}^{\perp}\left(-k_{2}+m\right) i \sigma_{\mu \nu}\left(k_{1}+m\right)\right]=4 m i\left[g_{\beta \mu}^{\perp} P_{\nu}-g_{\beta \nu}^{\perp} P_{\mu}\right] .
\end{aligned}
$$

Corresponding convolutions $B_{c}=\left(T_{c} T_{c}^{\prime}\right)$ read:

$$
\begin{aligned}
& \left(B_{S}\right)_{\beta^{\prime} \alpha^{\prime}}=64 m m^{\prime} k_{\beta^{\prime}} k_{\alpha^{\prime}}^{\prime}, \\
& \left(B_{V}\right)_{\beta^{\prime} \alpha^{\prime}}=4\left[s s^{\prime} g_{\beta^{\prime} \alpha^{\prime}}^{\perp}+4 s^{\prime} k_{\beta^{\prime}} k_{\alpha^{\prime}}+4 s k_{\beta^{\prime}}^{\prime} k_{\alpha^{\prime}}^{\prime}+16\left(k k^{\prime}\right) k_{\beta^{\prime}} k_{\alpha^{\prime}}^{\prime}\right], \\
& \left(B_{A}\right)_{\beta^{\prime} \alpha^{\prime}}=-16\left(P P^{\prime}\right)\left[k_{\beta^{\prime}}^{\prime} k_{\alpha^{\prime}}-\left(k k^{\prime}\right) g_{\beta^{\prime} \alpha^{\prime}}^{\perp}\right], \\
& \left(B_{T}\right)_{\beta^{\prime} \alpha^{\prime}}=32\left(P P^{\prime}\right) m m^{\prime} g_{\beta^{\prime} \alpha^{\prime}}^{\perp} .
\end{aligned}
$$

\section{APPENDIX C: APPENDIX: CONVOLUTIONS OF THE TRACE FACTORS}

Here we present the convolutions of the angular-momentum factors. We work with $k_{\mu}^{\perp}=k_{\nu} g_{\nu \mu}^{\perp}=k_{\mu}$ and $k_{\mu}^{\prime \perp}=$ $k_{\nu}^{\prime} g_{\nu \mu}^{\perp}=k_{\mu}^{\prime}$, and introduce:

$$
z=\frac{\left(k k^{\prime}\right)}{\sqrt{k^{2}} \sqrt{k^{\prime 2}}} .
$$

The convolutions for the $(S=1)$ states read:

$$
X_{\mu_{1} \mu_{2} \cdots \mu_{J}}^{(J)}(k) X_{\mu_{1} \mu_{2} \cdots \mu_{J}}^{(J)}\left(k^{\prime}\right)=\alpha(J)\left(\sqrt{k^{2}} \sqrt{k^{\prime 2}}\right)^{J} P_{J}(z) .
$$

Analogous convolutions for $(S=1)$-states are written as follows:

$$
\begin{aligned}
& X_{\mu_{1} \mu_{2} \cdots \mu_{J} \beta}^{(J+1)}(k) X_{\mu_{1} \mu_{2} \cdots \mu_{J} \alpha}^{(J+1)}\left(k^{\prime}\right)=\frac{\alpha(J)}{J+1}\left(\sqrt{k^{2}} \sqrt{k^{\prime 2}}\right)^{J} \times \\
& \times\left[\frac{\sqrt{k^{\prime 2}}}{\sqrt{k^{2}}} A_{P_{J, J+1}}(z) k_{\beta} k_{\alpha}+\frac{\sqrt{k^{2}}}{\sqrt{k^{\prime 2}}} B_{P_{J, J+1}}(z) k_{\beta}^{\prime} k_{\alpha}^{\prime}+\right. \\
& \left.+C_{P_{J, J+1}}(z) k_{\beta} k_{\alpha}^{\prime}+D_{P_{J, J+1}}(z) k_{\beta}^{\prime} k_{\alpha}+\left(\sqrt{k^{2}} \sqrt{k^{\prime 2}}\right) E_{P_{J, J+1}}(z) g_{\beta \alpha}^{\perp}\right] \\
& X_{\mu_{1} \mu_{2} \cdots \mu_{J} \beta}^{(J+1)}(k) Z_{\mu_{1} \mu_{2} \cdots \mu_{J}, \alpha}^{(J-1)}\left(k^{\prime}\right)=-\frac{\alpha(J)}{J} \frac{1}{k^{\prime 2}}\left(\sqrt{k^{2}} \sqrt{k^{\prime 2}}\right)^{J} \times \\
& \times\left[\frac{\sqrt{k^{\prime 2}}}{\sqrt{k^{2}}} A_{P_{J, J+1}}(z) k_{\beta} k_{\alpha}+\frac{\sqrt{k^{2}}}{\sqrt{k^{\prime 2}}}\left(B_{P_{J, J+1}}(z)-(2 J+1) A_{J}(z)\right) k_{\beta}^{\prime} k_{\alpha}^{\prime}+\right. \\
& +\left(C_{P_{J, J+1}}(z)-(2 J+1) B_{J}(z)\right) k_{\beta} k_{\alpha}^{\prime}+ \\
& \left.+D_{P_{J, J+1}}(z) k_{\beta}^{\prime} k_{\alpha}+\left(\sqrt{k^{2}} \sqrt{k^{\prime 2}}\right) E_{P_{J, J+1}}(z) g_{\beta \alpha}^{\perp}\right]
\end{aligned}
$$




$$
\begin{aligned}
& Z_{\mu_{1} \mu_{2} \cdots \mu_{J}, \beta}^{(J-1)}(k) Z_{\mu_{1} \mu_{2} \cdots \mu_{J}, \alpha}^{(J-1)}\left(k^{\prime}\right)=\frac{J+1}{J^{2}} \alpha(J)\left(\sqrt{k^{2}} \sqrt{k^{\prime 2}}\right)^{J-2} \times \\
\times & {\left[\frac{\sqrt{k^{\prime 2}}}{\sqrt{k^{2}}}\left(A_{P_{J, J+1}}(z)-(2 J+1) A_{J}(z)\right) k_{\beta} k_{\alpha}+\right.} \\
+ & \frac{\sqrt{k^{2}}}{\sqrt{k^{\prime 2}}}\left(B_{P_{J, J+1}}(z)-(2 J+1) A_{J}(z)\right) k_{\beta}^{\prime} k_{\alpha}^{\prime}+ \\
+ & \left(C_{P_{J, J+1}}(z)+\frac{(2 J+1)^{2}}{J+1} P_{J}(z)-2(2 J+1) B_{J}(z)\right) k_{\beta} k_{\alpha}^{\prime}+ \\
+ & \left.D_{P_{J, J+1}}(z) k_{\beta}^{\prime} k_{\alpha}+\left(\sqrt{k^{2}} \sqrt{k^{\prime 2}}\right) E_{P_{J, J+1}}(z) g_{\beta \alpha}^{\perp}\right], \\
& \epsilon_{\beta \nu_{1} \nu_{2} \nu_{3}} P_{\nu_{1}} Z_{\nu_{2} \mu_{1} \cdots \mu_{J}, \nu_{3}}^{(J)}(k) \epsilon_{\alpha \lambda_{1} \lambda_{2} \lambda_{3}} P_{\lambda_{1}}^{\prime} Z_{\lambda_{2} \mu_{1} \cdots \mu_{J}, \lambda_{3}}^{(J)}\left(k^{\prime}\right)= \\
& =\frac{(2 J+3)^{2}}{(J+1)^{3}} \alpha(J)\left(\sqrt{k^{2}} \sqrt{k^{\prime 2}}\right) \\
& \times\left[-\sqrt{k^{2}} \sqrt{k^{\prime 2}}\left(\left(z^{2}-1\right) D_{P_{J, J+1}}(z)+z P_{P_{J, J+1}}(z)\right) g_{\beta \alpha}^{\perp}-\right. \\
& -D_{P_{J, J+1}}(z)\left(\frac{\sqrt{k^{\prime 2}}}{\sqrt{k^{2}}} k_{\beta} k_{\alpha}+\frac{\sqrt{k^{2}}}{\sqrt{k^{\prime 2}}} k_{\beta}^{\prime} k_{\alpha}^{\prime}-z k_{\beta} k_{\alpha}^{\prime}\right)+ \\
& \left.+\left(z D_{P_{J, J+1}}(z)+E_{P_{J, J+1}}(z)\right) k_{\beta}^{\prime} k_{\alpha}\right],
\end{aligned}
$$

and

$$
\begin{aligned}
& X_{\mu_{1} \cdots \mu_{J}}^{(J)}(k) \epsilon_{\alpha \nu_{1} \nu_{2} \nu_{3}} P_{\nu_{1}}^{\prime} Z_{\nu_{2} \mu_{1} \cdots \mu_{J}, \nu_{3}}^{(J)}\left(k^{\prime}\right)= \\
& =\frac{2 J+3}{J+1} \alpha(J)\left(\sqrt{k^{2}} \sqrt{k^{\prime 2}}\right)^{J-1} A_{P_{J, J+1}}(z) \epsilon_{\alpha P^{\prime} k k^{\prime}}
\end{aligned}
$$

Here,

$$
\begin{aligned}
& A_{P_{J, J+1}}(z)= B_{P_{J, J+1}}(z)= \\
&=- \frac{2 z P_{J}(z)+\left[J z^{2}-(J+2)\right] P_{J+1}(z)}{\left(1-z^{2}\right)^{2}}, \\
& C_{P_{J, J+1}}(z)= \frac{\left[(1-J) z^{2}+(J+1)\right] P_{J}(z)}{\left(1-z^{2}\right)^{2}}+ \\
&+\frac{\left[(2 J+1) z^{2}-(2 J+3)\right] z P_{J+1}(z)}{\left(1-z^{2}\right)^{2}}, \\
& D_{P_{J, J+1}}(z)= \frac{\left[(J+2) z^{2}-J\right] P_{J}(z)-2 z P_{J+1}(z)}{\left(1-z^{2}\right)^{2}}, \\
& E_{P_{J, J+1}}(z)= \frac{z P_{J}(z)-P_{J+1}(z)}{\left(1-z^{2}\right)} . \\
& A_{J}=\frac{P_{J+1}(z)-z P_{J}(z)}{1-z^{2}}, \quad B_{J}=\frac{P_{J}(z)-z P_{J+1}(z)}{1-z^{2}}, \\
& \epsilon_{\alpha P^{\prime} k k^{\prime}}=\epsilon_{\alpha \beta \mu \nu} P^{\prime \beta} k^{\mu} k^{\prime \nu}
\end{aligned}
$$

We also need more complicated convolutions,namely, for the factors $K_{\beta} X_{\mu_{1} \mu_{2} \cdots \mu_{J} \beta}^{(J+1)}(k) X_{\mu_{1} \mu_{2} \cdots \mu_{J} \alpha}^{(J+1)}\left(k^{\prime}\right) K_{\alpha}$ where $K=k, k^{\prime}$ :

$$
\begin{aligned}
& k_{\beta} X_{\mu_{1} \mu_{2} \cdots \mu_{J} \beta}^{(J+1)}(k) X_{\mu_{1} \mu_{2} \cdots \mu_{J} \alpha}^{(J+1)}\left(k^{\prime}\right) k_{\alpha}= \\
& =k^{2} \alpha(J)\left(\sqrt{k^{2}} \sqrt{k^{\prime 2}}\right)^{J+1} P_{J+1}(z), \\
& k_{\beta} X_{\mu_{1} \mu_{2} \cdots \mu_{J} \beta}^{(J+1)}(k) X_{\mu_{1} \mu_{2} \cdots \mu_{J} \alpha}^{(J+1)}\left(k^{\prime}\right) k_{\alpha}^{\prime}=\alpha(J)\left(\sqrt{k^{2}} \sqrt{k^{\prime 2}}\right)^{J+2} P_{J}(z),
\end{aligned}
$$




$$
\begin{aligned}
& k_{\beta}^{\prime} X_{\mu_{1} \mu_{2} \cdots \mu_{J} \beta}^{(J+1)}(k) X_{\mu_{1} \mu_{2} \cdots \mu_{J} \alpha}^{(J+1)}\left(k^{\prime}\right) k_{\alpha}^{\prime}=k^{\prime 2} \alpha(J)\left(\sqrt{k^{2}} \sqrt{k^{\prime 2}}\right)^{J+1} P_{J+1}(z), \\
& k_{\beta}^{\prime} X_{\mu_{1} \mu_{2} \cdots \mu_{J} \beta}^{(J+1)}(k) X_{\mu_{1} \mu_{2} \cdots \mu_{J} \alpha}^{(J+1)}\left(k^{\prime}\right) k_{\alpha}= \\
& =\alpha(J)\left(\sqrt{k^{2}} \sqrt{k^{\prime 2}}\right)^{J+2}\left[\frac{2 J+1}{J+1} z P_{J+1}(z)-\frac{J}{J+1} P_{J}(z)\right], \\
& g_{\beta \alpha}^{\perp} X_{\mu_{1} \mu_{2} \cdots \mu_{J} \beta}^{(J+1)}(k) X_{\mu_{1} \mu_{2} \cdots \mu_{J} \alpha}^{(J+1)}\left(k^{\prime}\right)=\frac{2 J+1}{J+1} \alpha(J)\left(\sqrt{k^{2}} \sqrt{k^{\prime 2}}\right)^{J+1} P_{J+1}(z) ;
\end{aligned}
$$

and for the factors $\left(K_{\beta} X_{\mu_{1} \mu_{2} \cdots \mu_{J} \beta}^{(J+1)}(k) Z_{\mu_{1} \mu_{2} \cdots \mu_{J}, \alpha}^{(J-1)}\left(k^{\prime}\right) K_{\alpha}\right)$ :

$$
\begin{aligned}
& k_{\beta} X_{\mu_{1} \mu_{2} \cdots \mu_{J} \beta}^{(J+1)}(k) Z_{\mu_{1} \mu_{2} \cdots \mu_{J}, \alpha}^{(J-1)}\left(k^{\prime}\right) k_{\alpha}= \\
& =k^{4} \alpha(J)\left(\sqrt{k^{2}} \sqrt{k^{\prime 2}}\right)^{J-1} P_{J-1}(z), \\
& k_{\beta} X_{\mu_{1} \mu_{2} \cdots \mu_{J} \beta}^{(J+1)}(k) Z_{\mu_{1} \mu_{2} \cdots \mu_{J}, \alpha}^{(J-1)}\left(k^{\prime}\right) k_{\alpha}^{\prime}=k^{2} \alpha(J)\left(\sqrt{k^{2}} \sqrt{k^{\prime 2}}\right)^{J} P_{J}(z), \\
& k_{\beta}^{\prime} X_{\mu_{1} \mu_{2} \cdots \mu_{J} \beta}^{(J+1)}(k) Z_{\mu_{1} \mu_{2} \cdots \mu_{J}, \alpha}^{(J-1)}\left(k^{\prime}\right) k_{\alpha}^{\prime}=\alpha(J)\left(\sqrt{k^{2}} \sqrt{k^{\prime 2}}\right)^{J+1} P_{J+1}(z), \\
& k_{\beta}^{\prime} X_{\mu_{1} \mu_{2} \cdots \mu_{J} \beta}^{(J+1)}(k) Z_{\mu_{1} \mu_{2} \cdots \mu_{J}, \alpha}^{(J-1)}\left(k^{\prime}\right) k_{\alpha}=k^{2} \alpha(J)\left(\sqrt{k^{2}} \sqrt{k^{\prime 2}}\right)^{J} P_{J}(z), \\
& g_{\beta \alpha}^{\perp} X_{\mu_{1} \mu_{2} \cdots \mu_{J} \beta}^{(J+1)}(k) Z_{\mu_{1} \mu_{2} \cdots \mu_{J}, \alpha}^{(J-1)}\left(k^{\prime}\right)=0 ;
\end{aligned}
$$

and for the factors $\left(K_{\beta} Z_{\mu_{1} \mu_{2} \cdots \mu_{J}, \beta}^{(J-1)}(k) Z_{\mu_{1} \mu_{2} \cdots \mu_{J}, \alpha}^{(J-1)}\left(k^{\prime}\right) K_{\alpha}\right)$ :

$$
\begin{aligned}
& k_{\beta} Z_{\mu_{1} \mu_{2} \cdots \mu_{J}, \beta}^{(J-1)}(k) Z_{\mu_{1} \mu_{2} \cdots \mu_{J}, \alpha}^{(J-1)}\left(k^{\prime}\right) k_{\alpha}= \\
& =k^{2} \alpha(J)\left(\sqrt{k^{2}} \sqrt{k^{\prime 2}}\right)^{J-1} P_{J-1}(z), \\
& k_{\beta} Z_{\mu_{1} \mu_{2} \cdots \mu_{J}, \beta}^{(J-1)}(k) Z_{\mu_{1} \mu_{2} \cdots \mu_{J}, \alpha}^{(J-1)}\left(k^{\prime}\right) k_{\alpha}^{\prime}=\alpha(J)\left(\sqrt{k^{2}} \sqrt{k^{\prime 2}}\right)^{J} P_{J}(z), \\
& k_{\beta}^{\prime} Z_{\mu_{1} \mu_{2} \cdots \mu_{J}, \beta}^{(J-1)}(k) Z_{\mu_{1} \mu_{2} \cdots \mu_{J}, \alpha}^{(J-1)}\left(k^{\prime}\right) k_{\alpha}^{\prime}=k^{\prime 2} \alpha(J)\left(\sqrt{k^{2}} \sqrt{k^{\prime 2}}\right)^{J-1} P_{J-1}(z), \\
& k_{\beta}^{\prime} Z_{\mu_{1} \mu_{2} \cdots \mu_{J}, \beta}^{(J-1)}(k) Z_{\mu_{1} \mu_{2} \cdots \mu_{J}, \alpha}^{(J-1)}\left(k^{\prime}\right) k_{\alpha}= \\
& =\alpha(J)\left(\sqrt{k^{2}} \sqrt{k^{\prime 2}}\right)^{J}\left[\frac{2 J+1}{J} z P_{J-1}(z)-\frac{J+1}{J} P_{J}(z)\right], \\
& g_{\beta \alpha}^{\perp} Z_{\mu_{1} \mu_{2} \cdots \mu_{J}, \beta}^{(J-1)}(k) Z_{\mu_{1} \mu_{2} \cdots \mu_{J}, \alpha}^{(J-1)}\left(k^{\prime}\right)=\frac{2 J+1}{J} \alpha(J)\left(\sqrt{k^{2}} \sqrt{k^{\prime 2}}\right)^{J-1} P_{J-1}(z) ;
\end{aligned}
$$

and for the factors $\left(K_{\beta} \epsilon_{\beta \nu_{1} \nu_{2} \nu_{3}} P_{\nu_{1}} Z_{\nu_{2} \mu_{1} \cdots \mu_{J}, \nu_{3}}^{(J)}(k) \epsilon_{\alpha \lambda_{1} \lambda_{2} \lambda_{3}} P_{\lambda_{1}}^{\prime} \times\right.$

$$
\begin{aligned}
\times Z_{\lambda_{2} \mu_{1} \cdots \mu_{J}, \lambda_{3}}^{(J)}\left(k^{\prime}\right) & \left.K_{\alpha}\right): \\
& k_{\beta} \epsilon_{\beta \nu_{1} \nu_{2} \nu_{3}} P_{\nu_{1}} Z_{\nu_{2} \mu_{1} \cdots \mu_{J}, \nu_{3}}^{(J)}(k) \epsilon_{\alpha \lambda_{1} \lambda_{2} \lambda_{3}} P_{\lambda_{1}}^{\prime} Z_{\lambda_{2} \mu_{1} \cdots \mu_{J}, \lambda_{3}}^{(J)}\left(k^{\prime}\right) k_{\alpha}=0, \\
& k_{\beta} \epsilon_{\beta \nu_{1} \nu_{2} \nu_{3}} P_{\nu_{1}} Z_{\nu_{2} \mu_{1} \cdots \mu_{J}, \nu_{3}}^{(J)}(k) \epsilon_{\alpha \lambda_{1} \lambda_{2} \lambda_{3}} P_{\lambda_{1}}^{\prime} Z_{\lambda_{2} \mu_{1} \cdots \mu_{J}, \lambda_{3}}^{(J)}\left(k^{\prime}\right) k_{\alpha}^{\prime}=0, \\
& k_{\beta}^{\prime} \epsilon_{\beta \nu_{1} \nu_{2} \nu_{3}} P_{\nu_{1}} Z_{\nu_{2} \mu_{1} \cdots \mu_{J}, \nu_{3}}^{(J)}(k) \epsilon_{\alpha \lambda_{1} \lambda_{2} \lambda_{3}} P_{\lambda_{1}}^{\prime} Z_{\lambda_{2} \mu_{1} \cdots \mu_{J}, \lambda_{3}}^{(J)}\left(k^{\prime}\right) k_{\alpha}^{\prime}=0, \\
& k_{\beta}^{\prime} \epsilon_{\beta \nu_{1} \nu_{2} \nu_{3}} P_{\nu_{1}} Z_{\nu_{2} \mu_{1} \cdots \mu_{J}, \nu_{3}}^{(J)}(k) \epsilon_{\alpha \lambda_{1} \lambda_{2} \lambda_{3}} P_{\lambda_{1}}^{\prime} Z_{\lambda_{2} \mu_{1} \cdots \mu_{J}, \lambda_{3}}^{(J)}\left(k^{\prime}\right) k_{\alpha}= \\
& =\frac{(2 J+3)^{2}}{(J+1)^{3}} \alpha(J)\left(\sqrt{k^{2}} \sqrt{k^{\prime 2}}\right)^{J+1}\left(P P^{\prime}\right)\left[z P_{J}(z)-P_{J+1}(z)\right], \\
& g_{\beta \alpha}^{\perp} \epsilon_{\beta \nu_{1} \nu_{2} \nu_{3}} P_{\nu_{1}} Z_{\nu_{2} \mu_{1} \cdots \mu_{J}, \nu_{3}}^{(J)}(k) \epsilon_{\alpha \lambda_{1} \lambda_{2} \lambda_{3}} P_{\lambda_{1}}^{\prime} Z_{\lambda_{2} \mu_{1} \cdots \mu_{J}, \lambda_{3}}^{(J)}\left(k^{\prime}\right)= \\
& =-\frac{J(2 J+3)^{2}}{(J+1)^{3}} \alpha(J)\left(\sqrt{k^{2}} \sqrt{k^{\prime 2}}\right)^{J}\left(P P^{\prime}\right) P_{J}(z) .
\end{aligned}
$$




\section{APPENDIX D: APPENDIX: THE BETHE-SALPETER EQUATIONS FOR THE $\omega, \phi, A_{2}$ AND $F_{2}$ TRAJECTORIES}

Here we present the Bethe-Salpeter equations for the $\omega, \phi, a_{2}$, and $f_{2}$ trajectories. Though the explicit form of these equations is rather cumbersome, the investigation of these trajectories is informative for the reconstruction of quark-antiquark forces.

\section{Equations for the $a_{2}\left(M^{2}, n\right)$-trajectories}

The following states are located on two $\left(n, M^{2}\right)$-trajectories for the $a_{2}$ states $M \leq 2400 \mathrm{MeV}[15]$ :

1) $a_{2}(1320)$ with $n=1, a_{2}(1660)$ with $n=2, a_{2}(1950)$ with $n=3, a_{2}(2255)$ with $n=4$;

2) $a_{2}$-trajectory: $a_{2}(2030)$ with $n=1, a_{2}(2310)$ with $n=2$.

Correspondingly, we have two coupled equations for two wave functions:

$$
\begin{gathered}
\left(s-M^{2}\right)\left[4 \psi_{a_{2}, n}^{(1,1,2)}(s) A_{j}+\left(\frac{5}{3} s+4 k^{2}\right) \psi_{a_{2}, n}^{(1,3,2)}(s) B_{j}\right]= \\
=\int_{4 m^{2}}^{\infty} \frac{d s^{\prime}}{\pi} \rho\left(s^{\prime}\right) 8\left(-k^{\prime 2}\right) \psi_{a_{2}, n}^{(1,1,2)}\left(s^{\prime}\right) A_{j} \times \\
\times\left[\frac{5}{14}\left(s+\frac{12}{5} k^{2}\right) k^{\prime 4} \widetilde{V}_{V}^{(3)}\left(s, s^{\prime}\right)+\frac{3}{10} \sqrt{s s^{\prime}} k^{\prime 2} \widetilde{V}_{A}^{(2)}\left(s, s^{\prime}\right)-\right. \\
\left.-\frac{6}{5} m^{2} k^{\prime 2} \widetilde{V}_{S}^{(2)}\left(s, s^{\prime}\right)+\frac{1}{3}\left(s^{\prime}+\frac{8}{5} k^{\prime 2}\right) \widetilde{V}_{V}^{(1)}\left(s, s^{\prime}\right)\right]- \\
-\int_{\frac{4 m^{2}}{\pi}}^{\infty} \frac{d s^{\prime}}{\pi}\left(s^{\prime}\right) 2 k^{\prime 6} \psi_{a_{2}, n}^{(1,3,2)}\left(s^{\prime}\right) B_{j} \times \\
\times\left[\widetilde{V}_{V}^{(3)}\left(s, s^{\prime}\right)-m^{2} \frac{24}{5} \widetilde{V}_{S}^{(2)}\left(s, s^{\prime}\right)-\frac{4}{5} \sqrt{s s^{\prime}} \widetilde{V}_{A}^{(2)}\left(s, s^{\prime}\right)+\frac{32}{100} \sqrt{s s^{\prime}} \widetilde{V}_{T}^{(3)}\left(s, s^{\prime}\right)+\frac{5}{14}\left(\frac{5}{3} s s^{\prime}+4 s^{\prime} k^{2}+4 s k^{\prime 2}+\frac{48}{5} k^{2} k^{\prime 2}\right) \times\right.
\end{gathered}
$$

and

$$
\begin{gathered}
\left(s-M^{2}\right)\left[\left(\frac{5}{2} s+4 k^{2}\right) \psi_{a_{2}, n}^{(1,1,2)}(s) A_{j}+4 k^{4} \psi_{a_{2}, n}^{(1,3,2)}(s) B_{j}\right]= \\
=\int_{4 m^{2}}^{\infty} \frac{d s^{\prime}}{\pi} \rho\left(s^{\prime}\right) 2\left(-k^{\prime 2}\right) \psi_{a_{2}, n}^{(1,1,2)}\left(s^{\prime}\right) A_{j} \times \\
\times\left[m^{2} \frac{20}{3} \sqrt{s s^{\prime}} \widetilde{V}_{T}^{(1)}\left(s, s^{\prime}\right)+\frac{1}{3}\left(\frac{5}{2} s s^{\prime}+4 s^{\prime} k^{2}+4 s k^{\prime 2}+\frac{32}{5} k^{2} k^{\prime 2}\right) \widetilde{V}_{V}^{(1)}\left(s, s^{\prime}\right)-\right. \\
\left.-m^{2} \frac{24}{5} k^{2} k^{\prime 2} \widetilde{V}_{S}^{(2)}\left(s, s^{\prime}\right)-\frac{9}{5} \sqrt{s s^{\prime}} k^{2} k^{\prime 2} \widetilde{V}_{A}^{(2)}\left(s, s^{\prime}\right)+\frac{24}{7} k^{4} k^{\prime 4} \widetilde{V}_{V}^{(3)}\left(s, s^{\prime}\right)\right]-
\end{gathered}
$$




$$
\begin{gathered}
-\int_{4 m^{2}}^{\infty} \frac{d s^{\prime}}{\pi} \rho\left(s^{\prime}\right) 8 k^{\prime 6} \psi_{a_{2}, n}^{(1,3,2)}\left(s^{\prime}\right) B_{j} \times \\
\times\left[\frac{5}{14}\left(s^{\prime}+\frac{12}{5} k^{\prime 2}\right) k^{4} \widetilde{V}_{V}^{(3)}\left(s, s^{\prime}\right)+\frac{3}{10} \sqrt{s s^{\prime}} k^{2} \widetilde{V}_{A}^{(2)}\left(s, s^{\prime}\right)-\right. \\
\left.-m^{2} \frac{6}{5} k^{2} \widetilde{V}_{S}^{(2)}\left(s, s^{\prime}\right)+\frac{1}{3}\left(s+\frac{8}{5} k^{2}\right) \widetilde{V}_{V}^{(1)}\left(s, s^{\prime}\right)\right] .
\end{gathered}
$$

Normalization and orthogonality conditions read:

$$
\begin{gathered}
\int_{4 m^{2}}^{\infty} \frac{d s}{\pi} \rho(s)\left[A_{j}^{2}\left(\psi_{a_{2}, n}^{(1,1,2)}(s)\right)^{2} 2 \alpha(2)\left(-k^{2}\right)\left(\frac{5}{2} s+4 k^{2}\right)+\right. \\
+2 A_{j} B_{j} \psi_{a_{2}, n}^{(1,1,2)}(s) \psi_{a_{2}, n}^{(1,3,2)}(s) 8 \alpha(2)\left(-k^{6}\right)+ \\
\left.+B_{j}^{2}\left(\psi_{a_{2}, n}^{(1,3, J)}(s)\right)^{2} 2 \alpha(2)\left(-k^{6}\right)\left(\frac{5}{3} s+4 k^{2}\right)\right]=1
\end{gathered}
$$

and

$$
\begin{array}{r}
\int_{4 m^{2}}^{\infty} \frac{d s}{\pi} \rho(s)\left[A_{1} A_{2}\left(\psi_{a_{2}, n}^{(1,1,2)}(s)\right)^{2} 2 \alpha(2)\left(-k^{2}\right)\left(\frac{5}{2} s+4 k^{2}\right)+\right. \\
+\left(A_{1} B_{2}+A_{2} B_{1}\right) \psi_{a_{2}, n}^{(1,1,2)}(s) \psi_{a_{2}, n}^{(1,3,2)}(s) 8 \alpha(2)\left(-k^{6}\right)+ \\
\left.+B_{1} B_{2}\left(\psi_{a_{2}, n}^{(1,3,2)}(s)\right)^{2} 2 \alpha(2)\left(-k^{6}\right)\left(\frac{5}{3} s+4 k^{2}\right)\right]=0 .
\end{array}
$$

\section{Equations for the $\omega$ and $\phi\left(M^{2}, n\right)$-trajectories}

We have four trajectories in this sector with the following states located on the $\left(n, M^{2}\right)$-trajectories [15]:

1) $S$-wave dominant states: $\omega(780)$ with $n=1, \omega(1420)$ with $n=2, \omega(1800)$ with $n=3, \omega(2150)$ with $n=4$;

2) $D$-wave dominant states: $\omega(1640)$ with $n=1, \omega(1920)$ with $n=2, \omega(2295)$ with $n=3$;

3) $S$-wave dominant states: $\phi(1020)$ with $n=1, \phi(1660)$ with $n=2, \phi(1950)$ with $n=3$;

4) $D$-wave dominant states: $\phi(1700)$ with $n=1$.

Correspondingly, we have four coupled equations for four wave functions. The first one reads as follows:

$$
\begin{gathered}
\left(s-M^{2}\right)\left[4 \psi_{\omega,(n \bar{n}), n}^{(1,0,1)}(s) A_{j} \cos \Theta_{n}+\left(\frac{3}{2} s+4 k^{2}\right) \psi_{\omega,(n \bar{n}), n}^{(1,2,1)}(s) \times\right. \\
\left.\times B_{j} \cos \Theta_{n}^{\prime}\right]=\int_{4 m^{2}}^{\infty} \frac{d s^{\prime}}{\pi} \rho\left(s^{\prime}\right) 8 \psi_{\omega,(n \bar{n}), n}^{(1,0,1)}\left(s^{\prime}\right) A_{j} \cos \Theta_{n} \times \\
\times\left[\frac{3}{10}\left(s+\frac{8}{3} k^{2}\right) k^{\prime 4} \widetilde{V}_{(n \bar{n} \rightarrow n \bar{n}), V}^{(2)}\left(s, s^{\prime}\right)+\frac{1}{3} \sqrt{s s^{\prime}} k^{\prime 2} \widetilde{V}_{(n \bar{n} \rightarrow n \bar{n}), A}^{(1)}\left(s, s^{\prime}\right)-\right.
\end{gathered}
$$




$$
\begin{aligned}
& \left.-\frac{4}{3} m^{2} k^{\prime 2} \widetilde{V}_{(n \bar{n} \rightarrow n \bar{n}), S}^{(1)}\left(s, s^{\prime}\right)+\left(s^{\prime}+\frac{4}{3} k^{\prime 2}\right) \widetilde{V}_{(n \bar{n} \rightarrow n \bar{n}), V}^{(0)}\left(s, s^{\prime}\right)\right]+ \\
& +\int_{4 m^{2}}^{\infty} \frac{d s^{\prime}}{\pi} \rho\left(s^{\prime}\right) 2 k^{\prime 4} \psi_{\omega,(n \bar{n}), n}^{(1,2,1)}\left(s^{\prime}\right) B_{j} \cos \Theta_{n}^{\prime} \times \\
& \times\left[m^{2} \frac{18}{5} \sqrt{s s^{\prime}} \widetilde{V}_{(n \bar{n} \rightarrow n \bar{n}), T}^{(2)}\left(s, s^{\prime}\right)+\frac{3}{10}\left(\frac{3}{2} s s^{\prime}+4 s^{\prime} k^{2}+4 s k^{\prime 2}+\frac{32}{3} k^{2} k^{\prime 2}\right) \times\right. \\
& \times \widetilde{V}_{(n \bar{n} \rightarrow n \bar{n}), V}^{(2)}\left(s, s^{\prime}\right)-m^{2} \frac{16}{3} \widetilde{V}_{(n \bar{n} \rightarrow n \bar{n}), S}^{(1)}\left(s, s^{\prime}\right)-\frac{2}{3} \sqrt{s s^{\prime}} \widetilde{V}_{(n \bar{n} \rightarrow n \bar{n}), A}^{(1)}\left(s, s^{\prime}\right)+ \\
& \left.+\frac{16}{3} \widetilde{V}_{(n \bar{n} \rightarrow n \bar{n}), V}^{(0)}\left(s, s^{\prime}\right)\right]+\int_{4 m_{s}^{2}}^{\infty} \frac{d s^{\prime}}{\pi} \rho_{s}\left(s^{\prime}\right) 8 \psi_{\omega,(s \bar{s}), n}^{(1,0,1)}\left(s^{\prime}\right) A_{j} \sin \Theta_{n} \times \\
& \times\left[\frac{3}{10}\left(s+\frac{8}{3} k^{2}\right) k_{s}^{\prime 4} \widetilde{V}_{(s \bar{s} \rightarrow n \bar{n}), V}^{(2)}\left(s, s^{\prime}\right)+\frac{1}{3} \sqrt{s s^{\prime}} k_{s}^{\prime 2} \widetilde{V}_{(s \bar{s} \rightarrow n \bar{n}), A}^{(1)}\left(s, s^{\prime}\right)-\right. \\
& \left.-\frac{4}{3} m m_{s} k_{s}^{\prime 2} \widetilde{V}_{(s \bar{s} \rightarrow n \bar{n}), S}^{(1)}\left(s, s^{\prime}\right)+\left(s^{\prime}+\frac{4}{3} k_{s}^{\prime 2}\right) \widetilde{V}_{(s \bar{s} \rightarrow n \bar{n}), V}^{(0)}\left(s, s^{\prime}\right)\right]+ \\
& +\int_{4 m_{s}^{2}}^{\infty} \frac{d s^{\prime}}{\pi} \rho_{s}\left(s^{\prime}\right) 2 k_{s}^{\prime 4} \psi_{\omega,(s \bar{s}), n}^{(1,2,1)}\left(s^{\prime}\right) B_{j} \sin \Theta_{n}^{\prime} \times \\
& \times\left[m m_{s} \frac{18}{5} \sqrt{s s^{\prime}} \widetilde{V}_{(s \bar{s} \rightarrow n \bar{n}), T}^{(2)}\left(s, s^{\prime}\right)+\frac{3}{10}\left(\frac{3}{2} s s^{\prime}+4 s^{\prime} k^{2}+4 s k_{s}^{\prime 2}+\frac{32}{3} k^{2} k_{s}^{\prime 2}\right) \times\right. \\
& \times \widetilde{V}_{(s \bar{s} \rightarrow n \bar{n}), V}^{(2)}\left(s, s^{\prime}\right)-m m_{s} \frac{16}{3} \widetilde{V}_{(s \bar{s} \rightarrow n \bar{n}), S}^{(1)}\left(s, s^{\prime}\right)-\frac{2}{3} \sqrt{s s^{\prime}} \widetilde{V}_{(s \bar{s} \rightarrow n \bar{n}), A}^{(1)}\left(s, s^{\prime}\right)+ \\
& \left.+\frac{16}{3} \widetilde{V}_{(s \bar{s} \rightarrow n \bar{n}), V}^{(0)}\left(s, s^{\prime}\right)\right],
\end{aligned}
$$

the second one,

$$
\begin{gathered}
\left(s-M^{2}\right)\left[4 \psi_{\omega,(s \bar{s}), n}^{(1,0,1)}(s) A_{j} \sin \Theta_{n}+\left(\frac{3}{2} s+4 k_{s}^{2}\right) \psi_{\omega,(s \bar{s}), n}^{(1,2,1)}(s) \times\right. \\
\left.\times B_{j} \sin \Theta_{n}^{\prime}\right]=\int_{4 m^{2}}^{\infty} \frac{d s^{\prime}}{\pi} \rho\left(s^{\prime}\right) 8 \psi_{\omega,(n \bar{n}), n}^{(1,0,1)}\left(s^{\prime}\right) A_{j} \cos \Theta_{n} \times \\
\times\left[\frac{3}{10}\left(s+\frac{8}{3} k_{s}^{2}\right) k^{\prime 4} \widetilde{V}_{(n \bar{n} \rightarrow s \bar{s}), V}^{(2)}\left(s, s^{\prime}\right)+\frac{1}{3} \sqrt{s s^{\prime}} k^{\prime 2} \widetilde{V}_{(n \bar{n} \rightarrow s \bar{s}), A}^{(1)}\left(s, s^{\prime}\right)-\right.
\end{gathered}
$$




$$
\begin{aligned}
& \left.-\frac{4}{3} m m_{s} k^{\prime 2} \widetilde{V}_{(n \bar{n} \rightarrow s \bar{s}), S}^{(1)}\left(s, s^{\prime}\right)+\left(s^{\prime}+\frac{4}{3} k^{\prime 2}\right) \widetilde{V}_{(n \bar{n} \rightarrow s \bar{s}), V}^{(0)}\left(s, s^{\prime}\right)\right]+ \\
& +\int_{4 m^{2}}^{\infty} \frac{d s^{\prime}}{\pi} \rho\left(s^{\prime}\right) 2 k^{\prime 4} \psi_{\omega,(n \bar{n}), n}^{(1,2,1)}\left(s^{\prime}\right) B_{j} \cos \Theta_{n}^{\prime} \times \\
& \times\left[m m_{s} \frac{18}{5} \sqrt{s s^{\prime}} \widetilde{V}_{(n \bar{n} \rightarrow s \bar{s}), T}^{(2)}\left(s, s^{\prime}\right)+\frac{3}{10}\left(\frac{3}{2} s s^{\prime}+4 s^{\prime} k_{s}^{2}+4 s k^{\prime 2}+\frac{32}{3} k_{s}^{2} k^{\prime 2}\right) \times\right. \\
& \times \widetilde{V}_{(n \bar{n} \rightarrow s \bar{s}), V}^{(2)}\left(s, s^{\prime}\right)-m m_{s} \frac{16}{3} \widetilde{V}_{(n \bar{n} \rightarrow s \bar{s}), S}^{(1)}\left(s, s^{\prime}\right)-\frac{2}{3} \sqrt{s s^{\prime}} \widetilde{V}_{(n \bar{n} \rightarrow s \bar{s}), A}^{(1)}\left(s, s^{\prime}\right)+ \\
& \left.+\frac{16}{3} \widetilde{V}_{(n \bar{n} \rightarrow s \bar{s}), V}^{(0)}\left(s, s^{\prime}\right)\right]+\int_{4 m_{s}^{2}}^{\infty} \frac{d s^{\prime}}{\pi} \rho_{s}\left(s^{\prime}\right) 8 \psi_{\omega,(s \bar{s}), n}^{(1,0,1)}\left(s^{\prime}\right) A_{j} \sin \Theta_{n} \times \\
& \times\left[\frac{3}{10}\left(s+\frac{8}{3} k_{s}^{2}\right) k_{s}^{\prime 4} \widetilde{V}_{(s \bar{s} \rightarrow s \bar{s}), V}^{(2)}\left(s, s^{\prime}\right)+\frac{1}{3} \sqrt{s s^{\prime}} k_{s}^{\prime 2} \widetilde{V}_{(s \bar{s} \rightarrow s \bar{s}), A}^{(1)}\left(s, s^{\prime}\right)-\right. \\
& \left.-\frac{4}{3} m_{s}^{2} k_{s}^{\prime 2} \widetilde{V}_{(s \bar{s} \rightarrow s \bar{s}), S}^{(1)}\left(s, s^{\prime}\right)+\left(s^{\prime}+\frac{4}{3} k_{s}^{\prime 2}\right) \widetilde{V}_{(s \bar{s} \rightarrow s \bar{s}), V}^{(0)}\left(s, s^{\prime}\right)\right]+ \\
& +\int_{4 m_{s}^{2}}^{\infty} \frac{d s^{\prime}}{\pi} \rho_{s}\left(s^{\prime}\right) 2 k_{s}^{\prime 4} \psi_{\omega,(s \bar{s}), n}^{(1,2,1)}\left(s^{\prime}\right) B_{j} \sin \Theta_{n}^{\prime} \times \\
& \times\left[m_{s}^{2} \frac{18}{5} \sqrt{s s^{\prime}} \widetilde{V}_{(s \bar{s} \rightarrow s \bar{s}), T}^{(2)}\left(s, s^{\prime}\right)+\frac{3}{10}\left(\frac{3}{2} s s^{\prime}+4 s^{\prime} k_{s}^{2}+4 s k_{s}^{\prime 2}+\frac{32}{3} k_{s}^{2} k_{s}^{\prime 2}\right) \times\right. \\
& \times \widetilde{V}_{(s \bar{s} \rightarrow s \bar{s}), V}^{(2)}\left(s, s^{\prime}\right)-m_{s}^{2} \frac{16}{3} \widetilde{V}_{(s \bar{s} \rightarrow s \bar{s}), S}^{(1)}\left(s, s^{\prime}\right)-\frac{2}{3} \sqrt{s s^{\prime}} \widetilde{V}_{(s \bar{s} \rightarrow s \bar{s}), A}^{(1)}\left(s, s^{\prime}\right)+ \\
& \left.+\frac{16}{3} \widetilde{V}_{(s \bar{s} \rightarrow s \bar{s}), V}^{(0)}\left(s, s^{\prime}\right)\right]
\end{aligned}
$$

the third one,

$$
\begin{aligned}
& \left(s-M^{2}\right)\left[\left(3 s+4 k^{2}\right) \psi_{\omega,(n \bar{n}), n}^{(1,0,1)}(s) A_{j} \cos \Theta_{n}+4 k^{4} \psi_{\omega,(n \bar{n}), n}^{(1,2,1)}(s) \times\right. \\
& \left.\times B_{j} \cos \Theta_{n}^{\prime}\right]=\int_{4 m^{2}}^{\infty} \frac{d s^{\prime}}{\pi} \rho\left(s^{\prime}\right) 2 \psi_{\omega,(n \bar{n}), n}^{(1,0,1)}\left(s^{\prime}\right) A_{j} \cos \Theta_{n} \times \\
& \times\left[24 m^{2} \sqrt{s s^{\prime}} \widetilde{V}_{(n \bar{n} \rightarrow n \bar{n}), T}^{(0)}\left(s, s^{\prime}\right)+\left(3 s s^{\prime}+4 s^{\prime} k^{2}+4 s k^{\prime 2}+\frac{16}{3} k^{2} k^{\prime 2}\right) \times\right. \\
& \times \widetilde{V}_{(n \bar{n} \rightarrow n \bar{n}), V}^{(0)}\left(s, s^{\prime}\right)-m^{2} \frac{16}{3} k^{2} k^{\prime 2} \widetilde{V}_{(n \bar{n} \rightarrow n \bar{n}), S}^{(1)}\left(s, s^{\prime}\right)-\frac{8}{3} \sqrt{s s^{\prime}} k^{2} k^{\prime 2} \times
\end{aligned}
$$




$$
\begin{aligned}
& \left.\times \widetilde{V}_{(n \bar{n} \rightarrow n \bar{n}), A}^{(1)}\left(s, s^{\prime}\right)+\frac{16}{5} k^{4} k^{\prime 4} \widetilde{V}_{(n \bar{n} \rightarrow n \bar{n}), V}^{(2)}\left(s, s^{\prime}\right)\right]+\int_{4 m^{2}}^{\infty} \frac{d s^{\prime}}{\pi} \rho\left(s^{\prime}\right) 8 k^{\prime 4} \psi_{\omega,(n \bar{n}), n}^{(1,2,1)}\left(s^{\prime}\right) \times \\
& \times B_{j} \cos \Theta_{n}^{\prime}\left[\frac{3}{10}\left(s^{\prime}+\frac{8}{3} k^{\prime 2}\right) k^{4} \widetilde{V}_{(n \bar{n} \rightarrow n \bar{n}), V}^{(2)}\left(s, s^{\prime}\right)+\frac{1}{3} \sqrt{s s^{\prime}} k^{2} \widetilde{V}_{(n \bar{n} \rightarrow n \bar{n}), A}^{(1)}\left(s, s^{\prime}\right)-\right. \\
& \left.-m^{2} \frac{4}{3} k^{2} \widetilde{V}_{(n \bar{n} \rightarrow n \bar{n}), S}^{(1)}\left(s, s^{\prime}\right)+\left(s+\frac{4}{3} k^{2}\right) \widetilde{V}_{(n \bar{n} \rightarrow n \bar{n}), V}^{(0)}\left(s, s^{\prime}\right)\right]+ \\
& +\int_{4 m_{s}^{2}}^{\infty} \frac{d s^{\prime}}{\pi} \rho_{s}\left(s^{\prime}\right) 2 \psi_{\omega,(s \bar{s}), n}^{(1,0,1)}\left(s^{\prime}\right) A_{j} \sin \Theta_{n} \times \\
& \times\left[24 m m_{s} \sqrt{s s^{\prime}} \widetilde{V}_{(s \bar{s} \rightarrow n \bar{n}), T}^{(0)}\left(s, s^{\prime}\right)+\left(3 s s^{\prime}+4 s^{\prime} k^{2}+4 s k_{s}^{\prime 2}+\frac{16}{3} k^{2} k_{s}^{\prime 2}\right) \times\right. \\
& \times \widetilde{V}_{(s \bar{s} \rightarrow n \bar{n}), V}^{(0)}\left(s, s^{\prime}\right)-m m_{s} \frac{16}{3} k^{2} k_{s}^{\prime 2} \widetilde{V}_{(s \bar{s} \rightarrow n \bar{n}), S}^{(1)}\left(s, s^{\prime}\right)-\frac{8}{3} \sqrt{s s^{\prime}} k^{2} k_{s}^{\prime 2} \times \\
& \left.\times \widetilde{V}_{(s \bar{s} \rightarrow n \bar{n}), A}^{(1)}\left(s, s^{\prime}\right)+\frac{16}{5} k^{4} k_{s}^{\prime 4} \widetilde{V}_{(s \bar{s} \rightarrow n \bar{n}), V}^{(2)}\left(s, s^{\prime}\right)\right]+\int_{4 m_{s}^{2}}^{\infty} \frac{d s^{\prime}}{\pi} \rho_{s}\left(s^{\prime}\right) 8 k_{s}^{\prime 4} \psi_{\omega,(s \bar{s}), n}^{(1,2,1)}\left(s^{\prime}\right) \times \\
& \times B_{j} \sin \Theta_{n}^{\prime}\left[\frac{3}{10}\left(s^{\prime}+\frac{8}{3} k_{s}^{\prime 2}\right) k^{4} \widetilde{V}_{(s \bar{s} \rightarrow n \bar{n}), V}^{(2)}\left(s, s^{\prime}\right)+\frac{1}{3} \sqrt{s s^{\prime}} k^{2} \widetilde{V}_{(s \bar{s} \rightarrow n \bar{n}), A}^{(1)}\left(s, s^{\prime}\right)-\right. \\
& \left.-m m_{s} \frac{4}{3} k^{2} \widetilde{V}_{(s \bar{s} \rightarrow n \bar{n}), S}^{(1)}\left(s, s^{\prime}\right)+\left(s+\frac{4}{3} k^{2}\right) \widetilde{V}_{(s \bar{s} \rightarrow n \bar{n}), V}^{(0)}\left(s, s^{\prime}\right)\right],
\end{aligned}
$$

and the fourth equation:

$$
\begin{gathered}
\left(s-M^{2}\right)\left[\left(3 s+4 k_{s}^{2}\right) \psi_{\omega,(s \bar{s}), n}^{(1,0,1)}(s) A_{j} \sin \Theta_{n}+4 k_{s}^{4} \psi_{\omega,(s \bar{s}), n}^{(1,2,1)}(s) \times\right. \\
\left.\times B_{j} \sin \Theta_{n}^{\prime}\right]=\int_{4 m^{2}}^{\infty} \frac{d s^{\prime}}{\pi} \rho\left(s^{\prime}\right) 2 \psi_{\omega,(n \bar{n}), n}^{(1,0,1)}\left(s^{\prime}\right) A_{j} \cos \Theta_{n} \times \\
\times\left[24 m m_{s} \sqrt{s s^{\prime}} \widetilde{V}_{(n \bar{n} \rightarrow s \bar{s}), T}^{(0)}\left(s, s^{\prime}\right)+\left(3 s s^{\prime}+4 s^{\prime} k_{s}^{2}+4 s k^{\prime 2}+\frac{16}{3} k_{s}^{2} k^{\prime 2}\right) \times\right. \\
\times \widetilde{V}_{(n \bar{n} \rightarrow s \bar{s}), V}^{(0)}\left(s, s^{\prime}\right)-m m_{s} \frac{16}{3} k_{s}^{2} k^{\prime 2} \widetilde{V}_{(n \bar{n} \rightarrow s \bar{s}), S}^{(1)}\left(s, s^{\prime}\right)-\frac{8}{3} \sqrt{s s^{\prime}} k_{s}^{2} k^{\prime 2} \widetilde{V}_{(n \bar{n} \rightarrow s \bar{s}), A}^{(1)}\left(s, s^{\prime}\right)+ \\
\left.+\frac{16}{5} k_{s}^{4} k^{\prime 4} \widetilde{V}_{(n \bar{n} \rightarrow s \bar{s}), V}^{(2)}\left(s, s^{\prime}\right)\right]+\int_{4 m^{2}}^{\infty} \frac{d s^{\prime}}{\pi} \rho\left(s^{\prime}\right) 8 k^{\prime 4} \psi_{\omega,(n \bar{n}), n}^{(1,2,1)}\left(s^{\prime}\right) B_{j} \cos \Theta_{n}^{\prime} \times
\end{gathered}
$$




$$
\begin{aligned}
& \times\left[\frac{3}{10}\left(s^{\prime}+\frac{8}{3} k^{\prime 2}\right) k_{s}^{4} \widetilde{V}_{(n \bar{n} \rightarrow s \bar{s}), V}^{(2)}\left(s, s^{\prime}\right)+\frac{1}{3} \sqrt{s s^{\prime}} k_{s}^{2} \widetilde{V}_{(n \bar{n} \rightarrow s \bar{s}), A}^{(1)}\left(s, s^{\prime}\right)-\right. \\
& \left.-m m_{s} \frac{4}{3} k_{s}^{2} \widetilde{V}_{(n \bar{n} \rightarrow s \bar{s}), S}^{(1)}\left(s, s^{\prime}\right)+\left(s+\frac{4}{3} k_{s}^{2}\right) \widetilde{V}_{(n \bar{n} \rightarrow s \bar{s}), V}^{(0)}\left(s, s^{\prime}\right)\right]+ \\
& +\int_{4 m_{s}^{2}}^{\infty} \frac{d s^{\prime}}{\pi} \rho_{s}\left(s^{\prime}\right) 2 \psi_{\omega,(s \bar{s}), n}^{(1,0,1)}\left(s^{\prime}\right) A_{j} \sin \Theta_{n} \times \\
& \times\left[24 m_{s}^{2} \sqrt{s s^{\prime}} \widetilde{V}_{(s \bar{s} \rightarrow s \bar{s}), T}^{(0)}\left(s, s^{\prime}\right)+\left(3 s s^{\prime}+4 s^{\prime} k_{s}^{2}+4 s k_{s}^{\prime 2}+\frac{16}{3} k_{s}^{2} k_{s}^{\prime 2}\right) \times\right. \\
& \times \widetilde{V}_{(s \bar{s} \rightarrow s \bar{s}), V}^{(0)}\left(s, s^{\prime}\right)-m_{s}^{2} \frac{16}{3} k_{s}^{2} k_{s}^{\prime 2} \widetilde{V}_{(s \bar{s} \rightarrow s \bar{s}), S}^{(1)}\left(s, s^{\prime}\right)-\frac{8}{3} \sqrt{s s^{\prime}} k_{s}^{2} k_{s}^{\prime 2} \widetilde{V}_{(s \bar{s} \rightarrow s \bar{s}), A}^{(1)}\left(s, s^{\prime}\right)+ \\
& \left.+\frac{16}{5} k_{s}^{4} k_{s}^{\prime 4} \widetilde{V}_{(s \bar{s} \rightarrow s \bar{s}), V}^{(2)}\left(s, s^{\prime}\right)\right]+\int_{4 m_{s}^{2}}^{\infty} \frac{d s^{\prime}}{\pi} \rho_{s}\left(s^{\prime}\right) 8 k_{s}^{\prime 4} \psi_{\omega,(s \bar{s}), n}^{(1,2,1)}\left(s^{\prime}\right) B_{j} \sin \Theta_{n}^{\prime} \times \\
& \times\left[\frac{3}{10}\left(s^{\prime}+\frac{8}{3} k_{s}^{\prime 2}\right) k_{s}^{4} \widetilde{V}_{(s \bar{s} \rightarrow s \bar{s}), V}^{(2)}\left(s, s^{\prime}\right)+\frac{1}{3} \sqrt{s s^{\prime}} k_{s}^{2} \widetilde{V}_{(s \bar{s} \rightarrow s \bar{s}), A}^{(1)}\left(s, s^{\prime}\right)-\right. \\
& \left.-m_{s}^{2} \frac{4}{3} k_{s}^{2} \widetilde{V}_{(s \bar{s} \rightarrow s \bar{s}), S}^{(1)}\left(s, s^{\prime}\right)+\left(s+\frac{4}{3} k_{s}^{2}\right) \widetilde{V}_{(s \bar{s} \rightarrow s \bar{s}), V}^{(0)}\left(s, s^{\prime}\right)\right] .
\end{aligned}
$$

\section{Equations for the $f_{2}\left(n, M^{2}\right)$-trajectories}

The $f_{2}$ mesons lay on the following four trajectories in the $\left(n, M^{2}\right)$ plane [15]:

1) dominantly $P$-wave states: $f_{2}(1285)$ with $n=1, f_{2}(1640)$ at $n=2, f_{2}(1950)$ with $n=3, f_{2}(2210)$ with $n=4$;

2) dominantly $P$-wave states: $f_{2}(1525)$ with $n=1, f_{2}(1790)$ with $n=2$;

3) dominantly $F$-wave states: $f_{2}(2020)$ with $n=1, f_{2}(2290)$ with $n=2$;

4) dominantly $F$-wave state: $f_{2}(2200)$ with $n=1$.

We have four equations for these four sets of states:

the first one,

$$
\begin{gathered}
\left(s-M^{2}\right)\left[4 \psi_{f_{2},(n \bar{n}), n}^{(1,1,2)}(s) A_{j} \cos \Theta_{n}+\left(\frac{5}{3} s+4 k^{2}\right) \psi_{f_{2},(n \bar{n}), n}^{(1,3,2)}(s) \times\right. \\
\left.\times B_{j} \cos \Theta_{n}^{\prime}\right]=\int_{4 m^{2}}^{\infty} \frac{d s^{\prime}}{\pi} \rho\left(s^{\prime}\right) 8\left(-k^{\prime 2}\right) \psi_{f_{2},(n \bar{n}), n}^{(1,1,2)}\left(s^{\prime}\right) A_{j} \cos \Theta_{n} \times \\
\times\left[\frac{5}{14}\left(s+\frac{12}{5} k^{2}\right) k^{\prime 4} \widetilde{V}_{(n \bar{n} \rightarrow n \bar{n}), V}^{(3)}\left(s, s^{\prime}\right)+\frac{3}{10} \sqrt{s s^{\prime}} k^{\prime 2} \widetilde{V}_{(n \bar{n} \rightarrow n \bar{n}), A}^{(2)}\left(s, s^{\prime}\right)-\right. \\
\left.-\frac{6}{5} m^{2} k^{\prime 2} \widetilde{V}_{(n \bar{n} \rightarrow n \bar{n}), S}^{(2)}\left(s, s^{\prime}\right)+\frac{1}{3}\left(s^{\prime}+\frac{8}{5} k^{\prime 2}\right) \widetilde{V}_{(n \bar{n} \rightarrow n \bar{n}), V}^{(1)}\left(s, s^{\prime}\right)\right]-
\end{gathered}
$$




$$
\begin{aligned}
& -\int_{4 m^{2}}^{\infty} \frac{d s^{\prime}}{\pi} \rho\left(s^{\prime}\right) 2 k^{\prime 6} \psi_{f_{2},(n \bar{n}), n}^{(1,3) 2)}\left(s^{\prime}\right) B_{j} \cos \Theta_{n}^{\prime}\left[m^{2} \frac{100}{21} \sqrt{s s^{\prime}} \widetilde{V}_{(n \bar{n} \rightarrow n \bar{n}), T}^{(3)}\left(s, s^{\prime}\right)+\right. \\
& +\frac{5}{14}\left(\frac{5}{3} s s^{\prime}+4 s^{\prime} k^{2}+4 s k^{\prime 2}+\frac{48}{5} k^{2} k^{\prime 2}\right) \widetilde{V}_{(n \bar{n} \rightarrow n \bar{n}), V}^{(3)}\left(s, s^{\prime}\right)- \\
& \left.-m^{2} \frac{24}{5} \widetilde{V}_{(n \bar{n} \rightarrow n \bar{n}), S}^{(2)}\left(s, s^{\prime}\right)-\frac{4}{5} \sqrt{s s^{\prime}} \widetilde{V}_{(n \bar{n} \rightarrow n \bar{n}), A}^{(2)}\left(s, s^{\prime}\right)+\frac{32}{15} \widetilde{V}_{(n \bar{n} \rightarrow n \bar{n}), V}^{(1)}\left(s, s^{\prime}\right)\right]+ \\
& +\int_{4 m_{s}^{2}}^{\infty} \frac{d s^{\prime}}{\pi} \rho_{s}\left(s^{\prime}\right) 8\left(-k_{s}^{\prime 2}\right) \psi_{f_{2},(s \bar{s}), n}^{(1,1,2)}\left(s^{\prime}\right) A_{j} \sin \Theta_{n} \times \\
& \times\left[\frac{5}{14}\left(s+\frac{12}{5} k^{2}\right) k_{s}^{\prime 4} \widetilde{V}_{(s \bar{s} \rightarrow n \bar{n}), V}^{(3)}\left(s, s^{\prime}\right)+\frac{3}{10} \sqrt{s s^{\prime}} k_{s}^{\prime 2} \widetilde{V}_{(s \bar{s} \rightarrow n \bar{n}), A}^{(2)}\left(s, s^{\prime}\right)-\right. \\
& \left.-\frac{6}{5} m m_{s} k_{s}^{\prime 2} \widetilde{V}_{(s \bar{s} \rightarrow n \bar{n}), S}^{(2)}\left(s, s^{\prime}\right)+\frac{1}{3}\left(s^{\prime}+\frac{8}{5} k_{s}^{\prime 2}\right) \widetilde{V}_{(s \bar{s} \rightarrow n \bar{n}), V}^{(1)}\left(s, s^{\prime}\right)\right]- \\
& -\int_{4 m_{s}^{2}}^{\infty} \frac{d s^{\prime}}{\pi} \rho_{s}\left(s^{\prime}\right) 2 k_{s}^{\prime 6} \psi_{f_{2},(s \bar{s}), n}^{(1,3,2)}\left(s^{\prime}\right) B_{j} \sin \Theta_{n}^{\prime} \times \\
& \times\left[m m_{s} \frac{100}{21} \sqrt{s s^{\prime}} \widetilde{V}_{(s \bar{s} \rightarrow n \bar{n}), T}^{(3)}\left(s, s^{\prime}\right)+\frac{5}{14}\left(\frac{5}{3} s s^{\prime}+4 s^{\prime} k^{2}+4 s k_{s}^{\prime 2}+\frac{48}{5} k^{2} k_{s}^{\prime 2}\right) \times\right. \\
& \times \widetilde{V}_{(s \bar{s} \rightarrow n \bar{n}), V}^{(3)}\left(s, s^{\prime}\right)-m m_{s} \frac{24}{5} \widetilde{V}_{(s \bar{s} \rightarrow n \bar{n}), S}^{(2)}\left(s, s^{\prime}\right)-\frac{4}{5} \sqrt{s s^{\prime}} \widetilde{V}_{(s \bar{s} \rightarrow n \bar{n}), A}^{(2)}\left(s, s^{\prime}\right)+ \\
& \left.+\frac{32}{15} \widetilde{V}_{(s \bar{s} \rightarrow n \bar{n}), V}^{(1)}\left(s, s^{\prime}\right)\right]
\end{aligned}
$$

the second one,

$$
\begin{gathered}
\left(s-M^{2}\right)\left[4 \psi_{f_{2},(s \bar{s}), n}^{(1,1,2)}(s) A_{j} \sin \Theta_{n}+\left(\frac{5}{3} s+4 k_{s}^{2}\right) \psi_{f_{2},(s \bar{s}), n}^{(1,3,2)}(s) \times\right. \\
\left.\times B_{j} \sin \Theta_{n}^{\prime}\right]=\int_{4 m^{2}}^{\infty} \frac{d s^{\prime}}{\pi} \rho\left(s^{\prime}\right) 8\left(-k^{\prime 2}\right) \psi_{f_{2},(n \bar{n}), n}^{(1,1,2)}\left(s^{\prime}\right) A_{j} \cos \Theta_{n} \times \\
\times\left[\frac{5}{14}\left(s+\frac{12}{5} k_{s}^{2}\right) k^{\prime 4} \widetilde{V}_{(n \bar{n} \rightarrow s \bar{s}), V}^{(3)}\left(s, s^{\prime}\right)+\frac{3}{10} \sqrt{s s^{\prime}} k^{\prime 2} \widetilde{V}_{(n \bar{n} \rightarrow s \bar{s}), A}^{(2)}\left(s, s^{\prime}\right)-\right. \\
\left.-\frac{6}{5} m m_{s} k^{\prime 2} \widetilde{V}_{(n \bar{n} \rightarrow s \bar{s}), S}^{(2)}\left(s, s^{\prime}\right)+\frac{1}{3}\left(s^{\prime}+\frac{8}{5} k^{\prime 2}\right) \widetilde{V}_{(n \bar{n} \rightarrow s \bar{s}), V}^{(1)}\left(s, s^{\prime}\right)\right]-
\end{gathered}
$$




$$
\begin{aligned}
& -\int_{4 m^{2}}^{\infty} \frac{d s^{\prime}}{\pi} \rho\left(s^{\prime}\right) 2 k^{\prime 6} \psi_{f_{2},(n \bar{n}), n}^{(1,3,2)}\left(s^{\prime}\right) B_{j} \cos \Theta_{n}^{\prime}\left[m m_{s} \frac{100}{21} \sqrt{s s^{\prime}} \widetilde{V}_{(n \bar{n} \rightarrow s \bar{s}), T}^{(3)}\left(s, s^{\prime}\right)+\right. \\
& +\frac{5}{14}\left(\frac{5}{3} s s^{\prime}+4 s^{\prime} k_{s}^{2}+4 s k^{\prime 2}+\frac{48}{5} k_{s}^{2} k^{\prime 2}\right) \widetilde{V}_{(n \bar{n} \rightarrow s \bar{s}), V}^{(3)}\left(s, s^{\prime}\right)- \\
& \left.-m m_{s} \frac{24}{5} \widetilde{V}_{(n \bar{n} \rightarrow s \bar{s}), S}^{(2)}\left(s, s^{\prime}\right)-\frac{4}{5} \sqrt{s s^{\prime}} \widetilde{V}_{(n \bar{n} \rightarrow s \bar{s}), A}^{(2)}\left(s, s^{\prime}\right)+\frac{32}{15} \widetilde{V}_{(n \bar{n} \rightarrow s \bar{s}), V}^{(1)}\left(s, s^{\prime}\right)\right]+ \\
& +\int_{4 m_{s}^{2}}^{\infty} \frac{d s^{\prime}}{\pi} \rho_{s}\left(s^{\prime}\right) 8\left(-k_{s}^{\prime 2}\right) \psi_{f_{2},(s \bar{s}), n}^{(1,1,2)}\left(s^{\prime}\right) A_{j} \sin \Theta_{n} \times \\
& \times\left[\frac{5}{14}\left(s+\frac{12}{5} k_{s}^{2}\right) k_{s}^{\prime 4} \widetilde{V}_{(s \bar{s} \rightarrow s \bar{s}), V}^{(3)}\left(s, s^{\prime}\right)+\frac{3}{10} \sqrt{s s^{\prime}} k_{s}^{\prime 2} \widetilde{V}_{(s \bar{s} \rightarrow s \bar{s}), A}^{(2)}\left(s, s^{\prime}\right)-\right. \\
& \left.-\frac{6}{5} m_{s}^{2} k_{s}^{\prime 2} \widetilde{V}_{(s \bar{s} \rightarrow s \bar{s}), S}^{(2)}\left(s, s^{\prime}\right)+\frac{1}{3}\left(s^{\prime}+\frac{8}{5} k_{s}^{\prime 2}\right) \widetilde{V}_{(s \bar{s} \rightarrow s \bar{s}), V}^{(1)}\left(s, s^{\prime}\right)\right]- \\
& -\int_{4 m_{s}^{2}}^{\infty} \frac{d s^{\prime}}{\pi} \rho_{s}\left(s^{\prime}\right) 2 k_{s}^{\prime 6} \psi_{f_{2},(s \bar{s}), n}^{(1,3,2)}\left(s^{\prime}\right) B_{j} \sin \Theta_{n}^{\prime} \times \\
& \times\left[m_{s}^{2} \frac{100}{21} \sqrt{s s^{\prime}} \widetilde{V}_{(s \bar{s} \rightarrow s \bar{s}), T}^{(3)}\left(s, s^{\prime}\right)+\frac{5}{14}\left(\frac{5}{3} s s^{\prime}+4 s^{\prime} k_{s}^{2}+4 s k_{s}^{\prime 2}+\frac{48}{5} k_{s}^{2} k_{s}^{\prime 2}\right) \times\right. \\
& \times \widetilde{V}_{(s \bar{s} \rightarrow s \bar{s}), V}^{(3)}\left(s, s^{\prime}\right)-m_{s}^{2} \frac{24}{5} \widetilde{V}_{(s \bar{s} \rightarrow s \bar{s}), S}^{(2)}\left(s, s^{\prime}\right)-\frac{4}{5} \sqrt{s s^{\prime}} \widetilde{V}_{(s \bar{s} \rightarrow s \bar{s}), A}^{(2)}\left(s, s^{\prime}\right)+ \\
& \left.+\frac{32}{15} \widetilde{V}_{(s \bar{s} \rightarrow s \bar{s}), V}^{(s)}\left(s, s^{\prime}\right)\right]
\end{aligned}
$$

the third one,

$$
\begin{gathered}
\left(s-M^{2}\right)\left[\left(\frac{5}{2} s+4 k^{2}\right) \psi_{f_{2},(n \bar{n}), n}^{(1,1,2)}(s) A_{j} \cos \Theta_{n}+4 k^{4} \psi_{f_{2},(n \bar{n}), n}^{(1,3,2)}(s) \times\right. \\
\left.\times B_{j} \cos \Theta_{n}^{\prime}\right]=\int_{4 m^{2}}^{\infty} \frac{d s^{\prime}}{\pi} \rho\left(s^{\prime}\right) 2\left(-k^{\prime 2}\right) \psi_{f_{2},(n \bar{n}), n}^{(1,1,2)}\left(s^{\prime}\right) A_{j} \cos \Theta_{n} \times \\
\times\left[m^{2} \frac{20}{3} \sqrt{s s^{\prime}} \widetilde{V}_{(n \bar{n} \rightarrow n \bar{n}), T}^{(1)}\left(s, s^{\prime}\right)+\frac{1}{3}\left(\frac{5}{2} s s^{\prime}+4 s^{\prime} k^{2}+4 s k^{\prime 2}+\frac{32}{5} k^{2} k^{\prime 2}\right) \times\right. \\
\times \widetilde{V}_{(n \bar{n} \rightarrow n \bar{n}), V}^{(1)}\left(s, s^{\prime}\right)-m^{2} \frac{24}{5} k^{2} k^{\prime 2} \widetilde{V}_{(n \bar{n} \rightarrow n \bar{n}), S}^{(2)}\left(s, s^{\prime}\right)-\frac{9}{5} \sqrt{s s^{\prime}} k^{2} k^{\prime 2} \widetilde{V}_{(n \bar{n} \rightarrow n \bar{n}), A}^{(2)}\left(s, s^{\prime}\right)+
\end{gathered}
$$




$$
\begin{aligned}
& \left.+\frac{24}{7} k^{4} k^{\prime 4} \widetilde{V}_{(n \bar{n} \rightarrow n \bar{n}), V}^{(3)}\left(s, s^{\prime}\right)\right]-\int_{4 m^{2}}^{\infty} \frac{d s^{\prime}}{\pi} \rho\left(s^{\prime}\right) 8 k^{\prime 6} \psi_{f_{2},(n \bar{n}), n}^{(1,3,2)}\left(s^{\prime}\right) B_{j} \cos \Theta_{n}^{\prime} \times \\
& \times\left[\frac{5}{14}\left(s^{\prime}+\frac{12}{5} k^{\prime 2}\right) k^{4} \widetilde{V}_{(n \bar{n} \rightarrow n \bar{n}), V}^{(3)}\left(s, s^{\prime}\right)+\frac{3}{10} \sqrt{s s^{\prime}} k^{2} \widetilde{V}_{(n \bar{n} \rightarrow n \bar{n}), A}^{(2)}\left(s, s^{\prime}\right)-\right. \\
& \left.-m^{2} \frac{6}{5} k^{2} \widetilde{V}_{(n \bar{n} \rightarrow n \bar{n}), S}^{(2)}\left(s, s^{\prime}\right)+\frac{1}{3}\left(s+\frac{8}{5} k^{2}\right) \widetilde{V}_{(n \bar{n} \rightarrow n \bar{n}), V}^{(1)}\left(s, s^{\prime}\right)\right]+ \\
& +\int_{4 m_{s}^{2}}^{\infty} \frac{d s^{\prime}}{\pi} \rho_{s}\left(s^{\prime}\right) 2\left(-k_{s}^{\prime 2}\right)_{s} \psi_{f_{2},(s \bar{s}), n}^{(1,1,2)}\left(s^{\prime}\right) A_{j} \sin \Theta_{n}\left[m m_{s} \frac{20}{3} \sqrt{s s^{\prime}} \widetilde{V}_{(s \bar{s} \rightarrow n \bar{n}), T}^{(1)}\left(s, s^{\prime}\right)+\right. \\
& +\frac{1}{3}\left(\frac{5}{2} s s^{\prime}+4 s^{\prime} k^{2}+4 s k_{s}^{\prime 2}+\frac{32}{5} k^{2} k_{s}^{\prime 2}\right) \widetilde{V}_{(s \bar{s} \rightarrow n \bar{n}), V}^{(1)}\left(s, s^{\prime}\right)- \\
& -m m_{s} \frac{24}{5} k^{2} k_{s}^{\prime 2} \widetilde{V}_{(s \bar{s} \rightarrow n \bar{n}), S}^{(2)}\left(s, s^{\prime}\right)-\frac{9}{5} \sqrt{s s^{\prime}} k^{2} k_{s}^{\prime 2} \widetilde{V}_{(s \bar{s} \rightarrow n \bar{n}), A}^{(2)}\left(s, s^{\prime}\right)+ \\
& \left.+\frac{24}{7} k^{4} k_{s}^{\prime 4} \widetilde{V}_{(s \bar{s} \rightarrow n \bar{n}), V}^{(3)}\left(s, s^{\prime}\right)\right]-\int_{4 m_{s}^{2}}^{\infty} \frac{d s^{\prime}}{\pi} \rho_{s}\left(s^{\prime}\right) 8 k_{s}^{\prime 6} \psi_{f_{2},(s \bar{s}), n}^{(1,3,2)}\left(s^{\prime}\right) B_{j} \sin \Theta_{n}^{\prime} \times \\
& \times\left[\frac{5}{14}\left(s^{\prime}+\frac{12}{5} k_{s}^{\prime 2}\right) k^{4} \widetilde{V}_{(s \bar{s} \rightarrow n \bar{n}), V}^{(3)}\left(s, s^{\prime}\right)+\frac{3}{10} \sqrt{s s^{\prime}} k^{2} \widetilde{V}_{(s \bar{s} \rightarrow n \bar{n}), A}^{(2)}\left(s, s^{\prime}\right)-\right. \\
& \left.-m m_{s} \frac{6}{5} k^{2} \widetilde{V}_{(s \bar{s} \rightarrow n \bar{n}), S}^{(2)}\left(s, s^{\prime}\right)+\frac{1}{3}\left(s+\frac{8}{5} k^{2}\right) \widetilde{V}_{(s \bar{s} \rightarrow n \bar{n}), V}^{(1)}\left(s, s^{\prime}\right)\right],
\end{aligned}
$$

and the fourth equation,

$$
\begin{gathered}
\left(s-M^{2}\right)\left[\left(\frac{5}{2} s+4 k_{s}^{2}\right) \psi_{f_{2},(s \bar{s}), n}^{(1,1,2)}(s) A_{j} \sin \Theta_{n}+4 k_{s}^{4} \psi_{f_{2},(s \bar{s}), n}^{(1,3,2)}(s) \times\right. \\
\left.\times B_{j} \sin \Theta_{n}^{\prime}\right]=\int_{4 m^{2}}^{\infty} \frac{d s^{\prime}}{\pi} \rho\left(s^{\prime}\right) 2\left(-k^{\prime 2}\right) \psi_{f_{2},(n \bar{n}), n}^{(1,1,2)}\left(s^{\prime}\right) A_{j} \cos \Theta_{n} \times \\
\times\left[m m_{s} \frac{20}{3} \sqrt{s s^{\prime}} \widetilde{V}_{(n \bar{n} \rightarrow s \bar{s}), T}^{(1)}\left(s, s^{\prime}\right)+\frac{1}{3}\left(\frac{5}{2} s s^{\prime}+4 s^{\prime} k_{s}^{2}+4 s k^{\prime 2}+\frac{32}{5} k_{s}^{2} k^{\prime 2}\right) \times\right. \\
\times \widetilde{V}_{(n \bar{n} \rightarrow s \bar{s}), V}^{(1)}\left(s, s^{\prime}\right)-m m_{s} \frac{24}{5} k_{s}^{2} k^{\prime 2} \widetilde{V}_{(n \bar{n} \rightarrow s \bar{s}), S}^{(2)}\left(s, s^{\prime}\right)-\frac{9}{5} \sqrt{s s^{\prime}} k_{s}^{2} k^{\prime 2} \widetilde{V}_{(n \bar{n} \rightarrow s \bar{s}), A}^{(2)}\left(s, s^{\prime}\right)+ \\
\left.+\frac{24}{7} k_{s}^{4} k^{\prime 4} \widetilde{V}_{(n \bar{n} \rightarrow s \bar{s}), V}^{(3)}\left(s, s^{\prime}\right)\right]-\int_{4 m^{2}}^{\infty} \frac{d s^{\prime}}{\pi} \rho\left(s^{\prime}\right) 8 k^{\prime 6} \psi_{f_{2},(n \bar{n}), n}^{(1,3,2)}\left(s^{\prime}\right) B_{j} \cos \Theta_{n}^{\prime} \times
\end{gathered}
$$




$$
\begin{aligned}
& \times\left[\frac{5}{14}\left(s^{\prime}+\frac{12}{5} k^{\prime 2}\right) k_{s}^{4} \widetilde{V}_{(n \bar{n} \rightarrow s \bar{s}), V}^{(3)}\left(s, s^{\prime}\right)+\frac{3}{10} \sqrt{s s^{\prime}} k_{s}^{2} \widetilde{V}_{(n \bar{n} \rightarrow s \bar{s}), A}^{(2)}\left(s, s^{\prime}\right)-\right. \\
& \left.-m m_{s} \frac{6}{5} k_{s}^{2} \widetilde{V}_{(n \bar{n} \rightarrow s \bar{s}), S}^{(2)}\left(s, s^{\prime}\right)+\frac{1}{3}\left(s+\frac{8}{5} k_{s}^{2}\right) \widetilde{V}_{(n \bar{n} \rightarrow s \bar{s}), V}^{(1)}\left(s, s^{\prime}\right)\right]+ \\
& +\int_{4 m_{s}^{2}}^{\infty} \frac{d s^{\prime}}{\pi} \rho_{s}\left(s^{\prime}\right) 2\left(-k_{s}^{2}\right) \psi_{f_{2},(s \bar{s}), n}^{(1,1,2)}\left(s^{\prime}\right) A_{j} \sin \Theta_{n}\left[m_{s}^{2} \frac{20}{3} \sqrt{s s^{\prime}} \widetilde{V}_{(s \bar{s} \rightarrow s \bar{s}), T}^{(1)}\left(s, s^{\prime}\right)+\right. \\
& +\frac{1}{3}\left(\frac{5}{2} s s^{\prime}+4 s^{\prime} k_{s}^{2}+4 s k_{s}^{\prime 2}+\frac{32}{5} k_{s}^{2} k_{s}^{\prime 2}\right) \widetilde{V}_{(s \bar{s} \rightarrow s \bar{s}), V}^{(1)}\left(s, s^{\prime}\right)- \\
& -m_{s}^{2} \frac{24}{5} k_{s}^{2} k_{s}^{\prime 2} \widetilde{V}_{(s \bar{s} \rightarrow s \bar{s}), S}^{(2)}\left(s, s^{\prime}\right)-\frac{9}{5} \sqrt{s s^{\prime}} k_{s}^{2} k_{s}^{\prime 2} \widetilde{V}_{(s \bar{s} \rightarrow s \bar{s}), A}^{(2)}\left(s, s^{\prime}\right)+ \\
& \left.+\frac{24}{7} k_{s}^{4} k_{s}^{\prime 4} \widetilde{V}_{(s \bar{s} \rightarrow s \bar{s}), V}^{(3)}\left(s, s^{\prime}\right)\right]-\int_{4 m_{s}^{2}}^{\infty} \frac{d s^{\prime}}{\pi} \rho_{s}\left(s^{\prime}\right) 8 k_{s}^{\prime 6} \psi_{f_{2},(s \bar{s}), n}^{(1,3,2)}\left(s^{\prime}\right) B_{j} \sin \Theta_{n}^{\prime} \times \\
& \times\left[\frac{5}{14}\left(s^{\prime}+\frac{12}{5} k_{s}^{\prime 2}\right) k_{s}^{4} \widetilde{V}_{(s \bar{s} \rightarrow s \bar{s}), V}^{(3)}\left(s, s^{\prime}\right)+\frac{3}{10} \sqrt{s s^{\prime}} k_{s}^{2} \widetilde{V}_{(s \bar{s} \rightarrow s \bar{s}), A}^{(2)}\left(s, s^{\prime}\right)-\right. \\
& \left.-m_{s}^{2} \frac{6}{5} k_{s}^{2} \widetilde{V}_{(s \bar{s} \rightarrow s \bar{s}), S}^{(2)}\left(s, s^{\prime}\right)+\frac{1}{3}\left(s+\frac{8}{5} k_{s}^{2}\right) \widetilde{V}_{(s \bar{s} \rightarrow s \bar{s}), V}^{(1)}\left(s, s^{\prime}\right)\right]
\end{aligned}
$$

[1] E. Salpeter and H.A. Bethe, Phys. Rev. 84, 1232 (1951);

E. Salpeter, Phys. Rev. 91, 994 (1953).

[2] G.F. Chew, "The Analytic S-Matrix", (W.A. Benjamin, New York, 1966);

G.F. Chew and S. Mandelstam, Phys. Rev. 119, 467 (1960).

[3] V.V. Anisovich, M.N. Kobrinsky, D.I. Melikhov, and A.V. Sarantsev, Nucl. Phys. A544, 747 (1992).

[4] A.V. Anisovich and V.A. Sadovnikova, Yad. Fiz. 55, 2657 (1992); 57, 75 (1994); Eur. Phys. J. A2, 199 (1998).

[5] A.V. Anisovich and A.V. Sarantsev, Yad. Fiz. 55, 2163 (1992) [Sov. J. Nucl. Phys. 55, 1200 (1992)].

[6] A.V. Kapitanov, A.V. Sarantsev, Yad. Fiz. 56, 156 (1993).

[7] V.V. Anisovich, D.I. Melikhov, B.Ch. Metsch, H.R. Petry, Nucl. Phys. A563, 549 (1993).

[8] V.V. Anisovich, in "Hadron Spectroscopy and the Confinement Problem", Ed. by D.V. Bugg,( Plenum Press, New York, 1996).

[9] A.V. Anisovich, V.V. Anisovich, V.N. Markov, M.A. Matveev, V.A. Sarantsev, J. Phys. G. 28, 15 (2002).

[10] M.N. Kinzle-Focacci,in Proceedings of the VIIIth Blois Workshop, Protvino, Russia, 28 June-2 July 1999, ed. by V.A. Petrov, A.V. Prokudin, (World Scientific, 2000).

[11] V.A. Schegelsky, in Open Session of HEP Division of PNPI: "On the Eve of the XXI Century", 25-29 December 2000.

[12] CMD-2 Collab.(R.R. Akhmetshin et al.), Phys. Lett. B 462, 371 (1999); 462, 380 (1999);

SND Collab.(M.N. Achasov et al.), Phys. Lett. B 485, 349 (2000).

[13] V.V. Anisovich, D.I. Melikhov, V.A. Nikonov, Phys. Rev. D52, 5295 (1995); D55, 2918 (1997);

A.V. Anisovich, V.V. Anisovich, D.V. Bugg, and V.A. Nikonov, Phys. Lett. B456 80 (1999);

A.V. Anisovich, V.V. Anisovich, L. Montanet, and V.A. Nikonov, Eur. Phys. J. A6, 247 (1999). 
[14] A.V. Anisovich, V.V. Anisovich, and V.A. Nikonov, Eur. Phys. J. A12 103 (2001);

A.V. Anisovich, V.V. Anisovich, V.N. Markov, and V.A. Nikonov, Yad. Fiz., in press;

A.V. Anisovich, V.V. Anisovich, M.A. Matveev, and V.A. Nikonov, Yad. Fiz., in press.

[15] A.V. Anisovich, V.V. Anisovich, and A.V. Sarantsev, Phys. Rev. D 62, 051502 (2000);

A.V. Anisovich, V.V. Anisovich, V.A. Nikonov, and A.V. Sarantsev, in: "PNPI XXX, Scientific Highlights, Theoretical Physics Division",Gatchina (2001),p. 58;

V.V. Anisovich, "Systematics of $q \bar{q}$ states and glueball", hep-ph/ 0110326

[16] A.V. Anisovich, C.A. Baker, C.J. Batty et al., Phys. Lett. B449, 114 (1999); B452, 173 (1999); B452, 180 (1999); B452, 187 (1999); B472, 168 (2000); B476, 15 (2000); B477 , 19 (2000); B491, 40 (2000); B491, 47 (2000); B496, 145 (2000); B507, 23 (2001); B508, 6 (2001); B513, 281 (2001); B517, 261 (2001); B517, 273 (2001);

Nucl. Phys. A651, 253 (1999); A662, 319 (2000); A662, 344 (2000).

[17] PDG Group(D.E. Groom et al.), Eur. Phys. J. C 15, 1 (2000).

[18] R. Ricken, M. Koll, D. Merten, B.C. Metsch, and H.R. Petry, Eur. Phys. J. A 9, 221 (2000).

[19] R.D. Carlitz and D.B. Creamer, Ann. Phys. 118, 429 (1979);

E.V. Shuryak, Nucl. Phys. B203, 93 (1982);

V.V. Anisovich, S.M. Gerasyuta, and A.V. Sarantsev, Int. J. Mod. Phys. A6, 625 (1991).

[20] V.V. Anisovich and A.V. Sarantsev, hep-ph/0204328.

[21] L. Castillejo, F.J. Dyson,and R.H. Dalitz, Phys. Rev. 101, 453 (1956). 


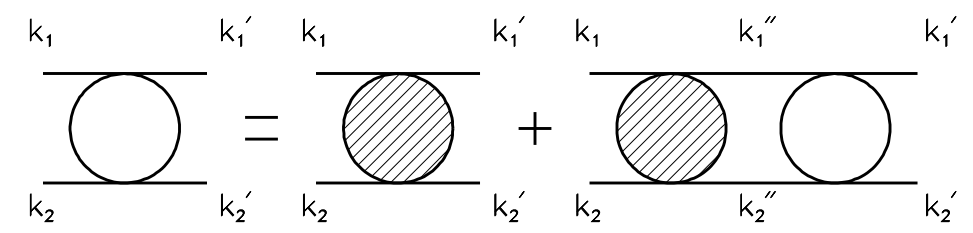

FIG. 1. Nonhomogeneous Bethe-Salpeter equation for the scattering amplitude; dashed block is the interaction kernel.

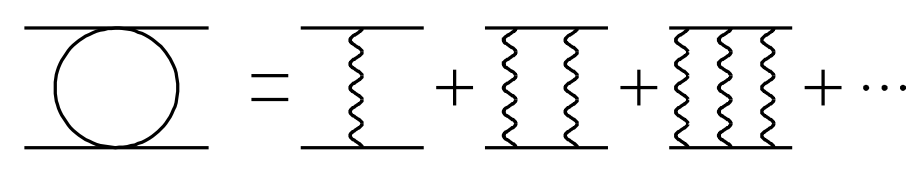

(a)

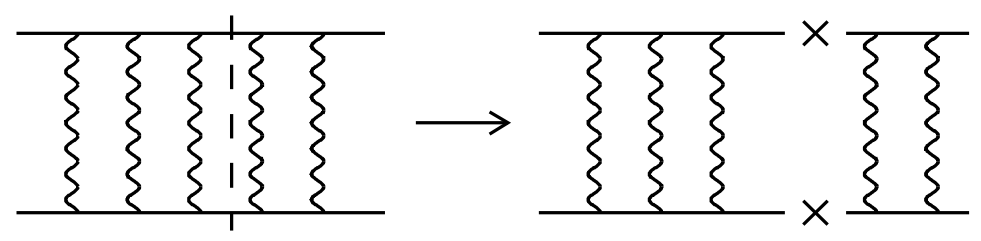

(b)

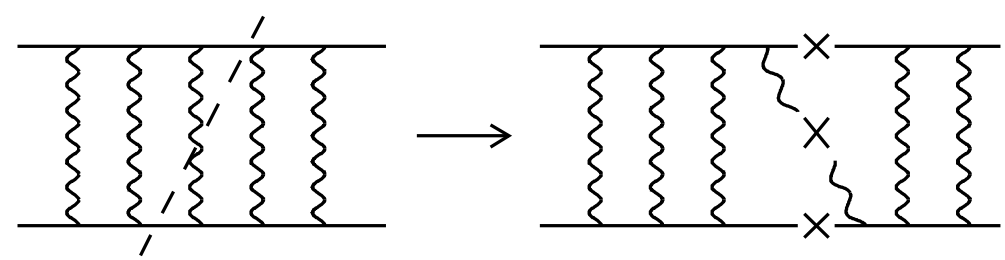

(c)

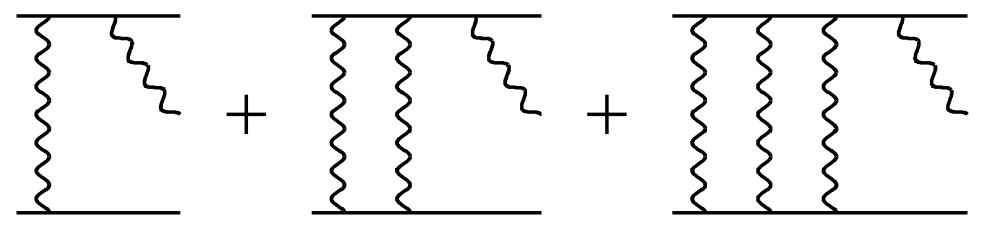

(d)

FIG. 2. a) Presentation of the scattering amplitude as a set of the ladder diagrams with the $t$-channel meson exchange interaction; b,c) cuttings of the ladder diagrams; d) meson production processes which are determined by ladder diagrams. 

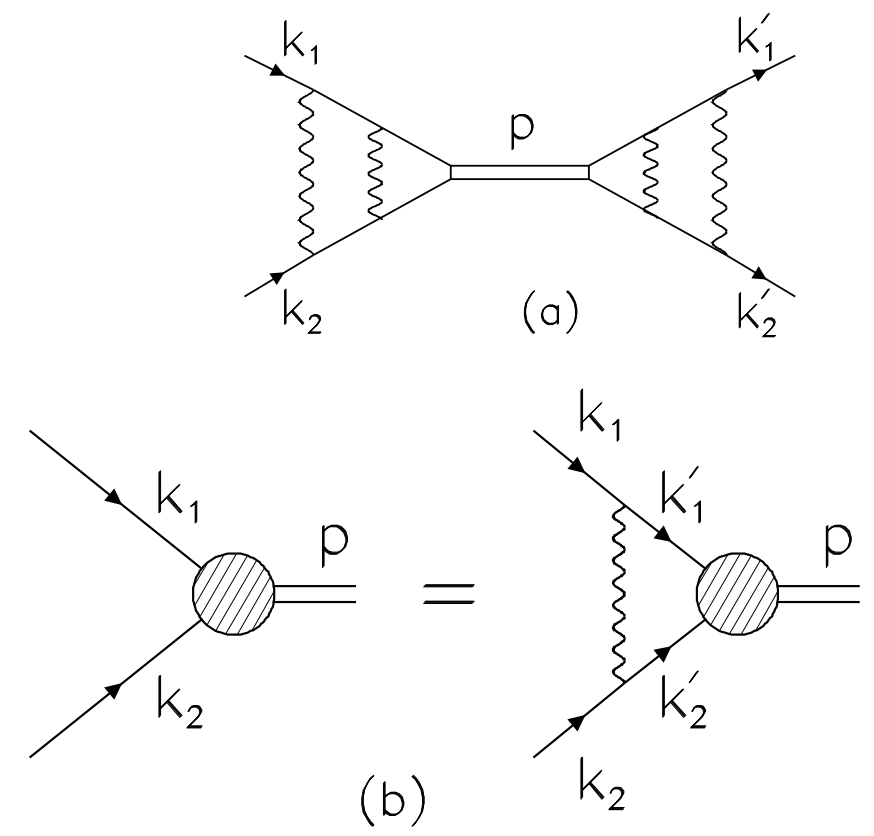

FIG. 3. a) Scattering amplitude near the pole related to the bound state; b) Bethe-Salpeter equation for the bound state

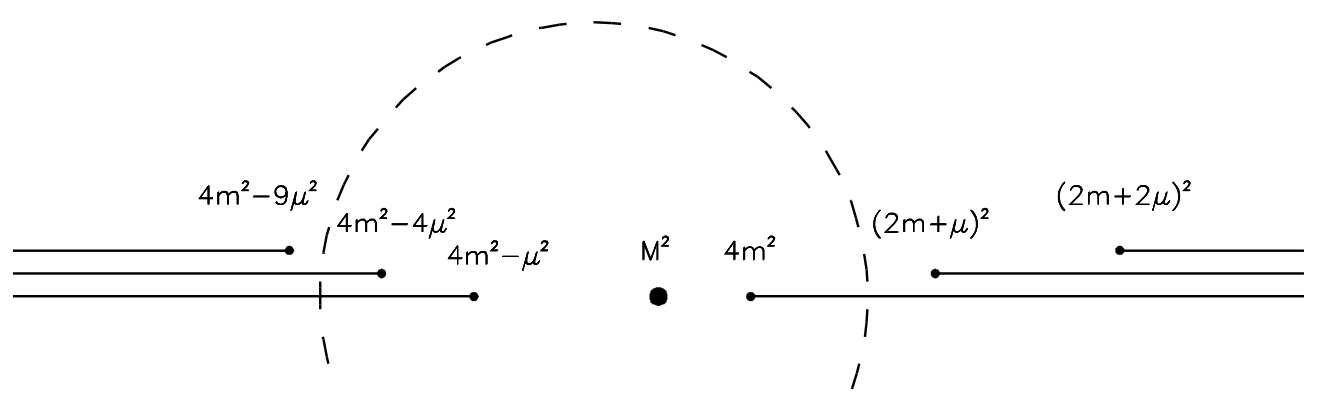

FIG. 4. The partial-wave amplitude singularities in the complex-s plane.

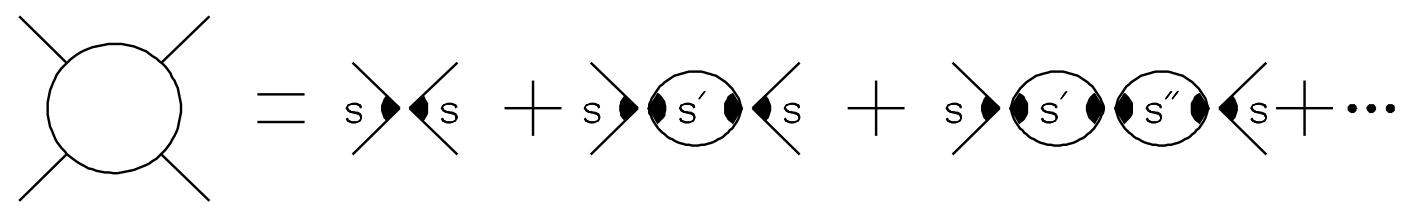

FIG. 5. Scattering amplitude in the dispersion-relation approach as a set of loop diagrams with separable vertices; there is no energy conservation in the intermediate states, $s \neq s^{\prime} \neq s^{\prime \prime}$, and so on. 